NBER WORKING PAPER SERIES

\title{
WELFARE AND OPTIMAL TRADING FREQUENCY IN DYNAMIC DOUBLE AUCTIONS
}

\author{
Songzi Du \\ Haoxiang Zhu
}

Working Paper 20588

http://www.nber.org/papers/w20588

\author{
NATIONAL BUREAU OF ECONOMIC RESEARCH \\ 1050 Massachusetts Avenue \\ Cambridge, MA 02138 \\ October 2014
}

\begin{abstract}
Earlier versions of this paper were circulated under the titles "Ex Post Equilibria in Double Auctions of Divisible Assets" and "Dynamic Ex Post Equilibrium, Welfare, and Optimal Trading Frequency in Double Auctions". For helpful comments, we are grateful to Alexis Berges, Bruno Biais, Alessandro Bonatti, Bradyn Breon-Drish, Jeremy Bulow, Giovanni Cespa, Hui Chen, David Dicks, Darrell Duffie, Thierry Foucault, Willie Fuchs, Lawrence Glosten, Lawrence Harris, Joel Hasbrouck, Terry Hendershott, Eiichiro Kazumori, Ilan Kremer, Martin Lettau, Stefano Lovo, Andrey Malenko, Katya Malinova, Gustavo Manso, Konstantin Milbradt, Sophie Moinas, Michael Ostrovsky, Jun Pan, Andreas Park, Parag Pathak, Michael Peters, Paul Pfleiderer, Uday Rajan, Marzena Rostek, Ioanid Rosu, Gideon Saar, Andy Skrzypacz, Chester Spatt, Juuso Toikka, Dimitri Vayanos, Jiang Wang, Yajun Wang, Bob Wilson, and Liyan Yang, Amir Yaron, Hayong Yun, as well as seminar participants at Stanford University, Simon Fraser University, MIT, Copenhagen Business School, University of British Columbia, UNC Junior Finance Faculty Roundtable, Midwest Theory Meeting, Finance Theory Group Berkeley Meeting, Canadian Economic Theory Conference, HEC Paris, Econometric Society Summer Meeting, Barcelona Information Workshop, CICF, Stony Brook Game Theory Festival, SAET, Bank of Canada, Carnegie Mellon Tepper, University of Toronto, NBER Microstructure meeting, IESE Business School, Stanford Institute for Theoretical Economics, UBC Summer Workshop in Economic Theory, Toulouse Conference on Trading in Electronic Market, University of Cincinnati, and the 10th Central Bank Workshop on the Microstructure of Financial Markets. The views expressed herein are those of the authors and do not necessarily reflect the views of the National Bureau of Economic Research.
\end{abstract}

NBER working papers are circulated for discussion and comment purposes. They have not been peerreviewed or been subject to the review by the NBER Board of Directors that accompanies official NBER publications.

(C) 2014 by Songzi Du and Haoxiang Zhu. All rights reserved. Short sections of text, not to exceed two paragraphs, may be quoted without explicit permission provided that full credit, including $(\mathbb{C}$ notice, is given to the source. 
Welfare and Optimal Trading Frequency in Dynamic Double Auctions

Songzi Du and Haoxiang Zhu

NBER Working Paper No. 20588

October 2014, Revised December 2015

JEL No. D44,D82,G14

\begin{abstract}
This paper studies the welfare consequence of increasing trading speed in financial markets. We build and solve a dynamic trading model, in which traders receive private information of asset value over time and trade strategically with demand schedules in a sequence of double auctions. A stationary linear equilibrium and its efficiency properties are characterized explicitly in closed form. Infrequent trading (few double auctions per unit of time) leads to a larger market depth in each trading period, but frequent trading allows more immediate asset re-allocation after new information arrives. Under natural conditions, the socially optimal trading frequency coincides with information arrival frequency for scheduled information releases, but can (far) exceed information arrival frequency for stochastic information arrivals. If traders have heterogeneous trading speeds, fast traders prefer the highest feasible trading frequency, whereas slow traders tend to prefer a strictly lower frequency.
\end{abstract}

\author{
Songzi Du \\ Department of Economics \\ 8888 University Drive \\ Burnaby, BC, Canada V5A 1S6 \\ songzid@sfu.ca \\ Haoxiang Zhu \\ MIT Sloan School of Management \\ 100 Main Street, E62-623 \\ Cambridge, MA 02142 \\ and NBER \\ zhuh@mit.edu
}




\title{
Welfare and Optimal Trading Frequency in Dynamic Double Auctions*
}

\author{
Songzi $\mathrm{Du}^{\dagger} \quad$ Haoxiang Zhu ${ }^{\ddagger}$
}

This version: December 22, 2015

First version: May 2012

\footnotetext{
*Earlier versions of this paper were circulated under the titles "Ex Post Equilibria in Double Auctions of Divisible Assets" and "Dynamic Ex Post Equilibrium, Welfare, and Optimal Trading Frequency in Double Auctions". For helpful comments, we are grateful to Alexis Bergès, Bruno Biais, Alessandro Bonatti, Bradyn Breon-Drish, Jeremy Bulow, Giovanni Cespa, Hui Chen, Peter DeMarzo, David Dicks, Darrell Duffie, Thierry Foucault, Willie Fuchs, Lawrence Glosten, Robin Greenwood, Lawrence Harris, Joel Hasbrouck, Terry Hendershott, Eiichiro Kazumori, Ilan Kremer, Pete Kyle, Martin Lettau, Stefano Lovo, Andrey Malenko, Katya Malinova, Gustavo Manso, Konstantin Milbradt, Sophie Moinas, Michael Ostrovsky, Jun Pan, Andreas Park, Jonathan Parker, Parag Pathak, Michael Peters, Paul Pfleiderer, Uday Rajan, Marzena Rostek, Ioanid Rosu, Gideon Saar, Xianwen Shi, Andy Skrzypacz, Chester Spatt, Juuso Toikka, Dimitri Vayanos, Xavier Vives, Jiang Wang, Yajun Wang, Bob Wilson, Liyan Yang, Amir Yaron, and Hayong Yun, as well as seminar participants at Stanford University, Simon Fraser University, MIT, Copenhagen Business School, University of British Columbia, UNC Junior Finance Faculty Roundtable, Midwest Theory Meeting, Finance Theory Group Berkeley Meeting, Canadian Economic Theory Conference, HEC Paris, Barcelona Information Workshop, CICF, Stony Brook Game Theory Festival, SAET, Bank of Canada, Carnegie Mellon Tepper, University of Toronto, NBER Microstructure meeting, IESE Business School, Stanford Institute for Theoretical Economics, UBC Summer Workshop in Economic Theory, Toulouse Conference on Trading in Electronic Market, University of Cincinnati, the 10th Central Bank Workshop on the Microstructure of Financial Markets, FIRN Asset Pricing meeting, Imperial College High Frequency Trading Conference, UPenn Workshop on Multiunit Allocation, WFA, Econometric Society World Congress, and the National University of Singapore. Paper URL: http://ssrn.com/abstract=2040609.

${ }^{\dagger}$ Simon Fraser University, Department of Economics, 8888 University Drive, Burnaby, B.C. Canada, V5A 1S6. songzid@sfu.ca.

${ }^{\ddagger}$ MIT Sloan School of Management and NBER, 100 Main Street E62-623, Cambridge, MA 02142. zhuh@mit.edu.
} 


\section{Introduction}

Trading in financial markets has become significantly faster over the last decade. Today, electronic transactions for equities, futures, and foreign exchange are typically conducted within millisecond or microseconds. Electronic markets, which typically have a higher speed than manual markets, are also increasingly adapted in the over-the-counter markets for debt securities and derivatives, such as corporate bonds, interest rates swaps, and credit default swaps. Exchange traded funds, which trade at a high frequency like stocks, have gained significant market shares over index mutual funds, which only allow buying and selling at the end of day.

The remarkable speedup in financial markets raises important economic questions. For example, does a higher speed of trading necessarily lead to a higher social welfare, in terms of more efficient allocations of assets? What is the socially optimal frequency (if one exists) at which financial markets should operate? Moreover, given that certain investors trade at a higher speed than others, does a higher trading frequency affect fast investors and slow ones equally or differentially? Answers to these questions would provide valuable insights for the ongoing academic and policy debate on market structure, especially in the context of high-speed trading (see, for example, Securities and Exchange Commission (2010)).

In this paper, we set out to investigate the welfare consequence of speeding up trading in financial markets. We build and solve a dynamic model with strategic trading, adverse selection, and imperfect competition. Specifically, in our model, a finite number $(n \geq 3)$ of traders trade a divisible asset in an infinite sequence of uniform-price double auctions, held at discrete time intervals. The shorter is the time interval between auctions, the higher is the speed of the market. At an exponentially-distributed time in the future, the asset pays a liquidating dividend, which, until that payment time, evolves according to a jump process. Over time, traders receive private, informative signals of common dividend shocks, as well as idiosyncratic shocks to their private values for owning the asset. Traders' values for the assets are therefore interdependent, creating adverse selection in the trading process. ${ }^{1}$ Traders also incur quadratic costs for holding inventories, which is equivalent to linearly decreasing marginal values. A trader's dividend signals, shocks to his private values, and his inventories are all his private information. In each double auction, traders submit demand schedules (i.e., a set of limit orders) and pay for their allocations at the market-clearing price. All traders take into account the price impact of their trades and are forward-looking about future trading opportunities.

Our model incorporates many salient features of dynamic markets in practice. For example, asymmetric and dispersed information about the common dividend creates adverse selection,

\footnotetext{
${ }^{1}$ Throughout this paper, "adverse selection" covers situations in which different traders have different pieces of information regarding the same asset. In our context "adverse selection" may also be read as "interdependent values."
} 
whereas private-value information and convex inventory costs introduce gains from trade. These trading motives are also time-varying as news arrives over time. Moreover, the number of double auctions per unit of clock time is a simple yet realistic way to model trading frequency in dynamic markets.

A dynamic equilibrium and efficiency. Our first main result is the characterization of a linear stationary equilibrium in this dynamic market, as well as its efficiency properties. In equilibrium, a trader's optimal demand in each double auction is a linear function of the price, his signal of the dividend, his most recent private value, and his private inventory. Each coefficient is solved explicitly in closed form. Naturally, the equilibrium price in each auction is a weighted sum of the average signal of the common dividend and the average private value, adjusted for the marginal holding cost of the average inventory. Prices are martingales since the innovations in common dividend and private values have zero mean.

Because there are a finite number of traders, demand schedules in this dynamic equilibrium are not competitive. Consequently, the equilibrium allocations of assets across traders after each auction are not fully efficient, but they converge gradually and exponentially over time to the efficient allocation. This convergence remains slow and gradual even in the continuous-time limit. (In reality, slow trading of this sort means splitting a large order into many smaller pieces and executing them over time.) We show that the convergence rate per unit of clock time increases with the number of traders, the arrival intensity of the dividend, the variance of the private-value shocks, and the trading frequency of the market; but the convergence rate decreases with the variance of the common-value shocks, which is a measure of adverse selection.

Welfare and optimal trading frequency. Our modeling framework proves to be an effective tool in answering welfare questions. Characterizing welfare and optimal trading frequency in this dynamic market is the second primary contribution of our paper.

We ask two related questions regarding trading frequency. First, what is the socially optimal trading frequency if all traders have equal speed? Second, if certain traders are faster than others, what are the trading frequencies that are optimal for fast and slow traders respectively?

Homogeneous speed. The first question on homogenous speed can be readily analyzed in our benchmark model, in which all traders participate in all double auctions. We emphasize that a change of trading frequency in our model does not change the fundamental properties of the asset, such as the timing and magnitude of the dividend shocks.

Increasing trading frequency involves the following important tradeoff. On the one hand, a higher trading frequency allows traders to react more quickly to new information and to trade sooner toward the efficient allocation. This effect favors a faster market. On the other hand, a 
lower trading frequency serves as a commitment device that induces more aggressive demand schedules (i.e., buy and sell orders are less sensitive to prices), which leads to more efficient allocations in early rounds of trading. This effect favors a slower market. Analytically, the allocative inefficiency in this dynamic market relative to the first best can be decomposed into two components: one part due to strategic behavior and the other due to the delayed responses to new information. The optimal trading frequency should strike the best balance between maximizing the aggressiveness of demand schedules and minimizing delays in reacting to new information.

We show that depending on the nature of information arrivals, this tradeoff leads to different optimal trading frequencies. If new information of dividend and private values arrives at scheduled time intervals, the optimal trading frequency cannot be higher than the frequency of new information. In the natural case that all traders are ex-ante identical, the optimal trading frequency coincides with the information arrival frequency. Intuitively, if information arrival times are known in advance, aligning trading times with information arrival times would reap all the benefit of immediate response to new information, while maximizing the average aggressiveness of demand schedules.

By contrast, if new information arrives at Poisson times, which are unpredictable, it is important to keep the market open more often to prevent excessive delays in responding to new information. Indeed, we show that with ex-ante identical traders and under Poisson information arrivals, the optimal trading frequency is always higher than two thirds of the information arrival frequency. Moreover, we show explicit conditions under which the optimal trading frequency is bounded below by a constant multiple of the information arrival frequency. This lower-bound multiple is larger if adverse selection is less of a concern. In the special case without adverse selection, the optimal trading frequency is at least $n / 2$ times the information arrival frequency. In the limit, as the number of traders $n$ becomes large or as the arrival rate of information goes to infinity, continuous trading becomes optimal. ${ }^{2}$ These results suggest that the optimal trading frequency for a particular asset should roughly increase in the "liquidity" of the asset.

Heterogeneous speeds. To answer the second question of welfare, we extend the model to allow heterogeneous trading speeds. In this extension, fast traders access the market whenever it is open, but slow traders only access the market periodically with a delay. This implies that fast traders participate in all double auctions, but each slow trader only participates in a fraction of the auctions. Different from recent studies of high-frequency trading (see the literature section), fast traders have no information advantage over slow ones.

We find that fast and slow traders generally prefer different frequencies at which the market

\footnotetext{
${ }^{2}$ To clarify, continuous trading in our model means continuous double auctions, not a continuous limit order book. The latter is effectively a discriminatory-price auction, not a uniform-price auction.
} 
operates. By staying in the market all the time, fast traders in our model play the endogenous role of market-makers: intermediating trades among slow traders who arrive sequentially. Through intermediation fast traders extract rents. A higher trading frequency reduces the number of slow traders in each double auction, making the market "thinner" and fast traders' rents higher. We show that fast traders prefer the highest trading frequency (i.e. the thinnest market) that is feasible. By contrast, slow traders typically prefer a strictly lower trading frequency (and a thicker market) because they benefit from pooling trading interests over time and providing liquidity to each other, even though a lower trading frequency implies a higher average delay cost for them. A broad implication from this analysis is that who designs the market matters a great deal for everyone.

Another direct observation from the heterogeneous-speed model is that, due to imperfect competition and market power, a higher trading frequency creates larger and more abrupt price reactions to supply and demand shocks (i.e. "price overshooting"), as well as subsequent reversals. These price paths exhibit high short-term volatility and resemble (mini) "flash crashes" and "flash rallies" observed in electronic markets.

Relation to the literature. The paper closest to ours is Vayanos (1999), who studies a dynamic market in which the asset fundamental value (dividend) is public information, but agents receive periodic private inventory shocks. Traders in his model also face imperfect competition and strategically avoid price impact. Vayanos (1999) shows that, if inventory shocks are small, then a lower trading frequency is better for welfare by encouraging traders to submit more aggressive demand schedules. ${ }^{3}$ We make two main contributions relative to Vayanos (1999). First, our model allows interdependent values and adverse selection. Adverse selection makes trading less aggressive and reduces the optimal trading frequency. Second, our model identifies two channels of welfare losses: One channel, strategic behavior, agrees with Vayanos (1999), whereas the other, delayed responses to news, complements Vayanos (1999). The latter channel is absent in Vayanos (1999) because inventory shocks and trading times always coincide in his model. We show that the latter channel can lead to an optimal trading frequency that is much faster than information arrival frequency if information arrival is stochastic. Our result also generates useful predictions regarding how the optimal trading frequency varies with asset characteristics.

In another related paper, Rostek and Weretka (2015) study dynamic trading with multiple dividend payments. In their model, traders have symmetric information about the asset's

\footnotetext{
${ }^{3}$ Vayanos (1999) also shows that if inventory information is common knowledge, there is a continuum of equilibria. Under one of these equilibria, selected by a trembling hand refinement, welfare is increasing in trading frequency. Because our model has private information of inventories, the private-information equilibrium of Vayanos (1999) is a more appropriate benchmark for comparison.
} 
fundamental value, and between consecutive dividend payments there is no news and no discounting. In this setting, they show that a higher trading frequency is better for welfare. Our contribution relative to Rostek and Weretka (2015) is similar to that relative to Vayanos (1999). First, our model applies to markets with adverse selection. Second, we show that the optimal trading frequency can be slow or fast, depending on the tradeoff between strategic behaviors and delayed responses to news.

Among recent models that study dynamic trading with adverse selection, the closest one to ours is Kyle, Obizhaeva, and Wang (2014). They study a continuous-time trading model in which agents have pure common values but "agree to disagree" on the precision of their signals. Although the disagreement component in their model and the private-value component in ours appear equivalent, they are in fact very different. As highlighted by Kyle, Obizhaeva, and Wang (2014), in a disagreement model the traders disagree not only about their values today, but also about how the values evolve over time; this behavior does not show up in a private-value model. Therefore, their model and ours answer very different economic questions: Their model generates "beauty contest" and non-martingale price dynamics, whereas our model is useful for characterizing the optimal trading frequency.

The last part of our paper on heterogeneous trading speed is most related to the model by Duffie (2010), who proposes an inattention-based model to explain asset price behaviors around large supply or demand shocks. The inattentive and attentive investors in his model correspond to the slow and fast traders in our model. Going beyond Duffie (2010), our model has imperfect competition, so price reactions to supply and demand shocks reflect market power and are hence more volatile. Moreover, from a welfare viewpoint, we find that fast traders in fact prefer the most volatile, highest-frequency market, because that is where they make the highest intermediation profits.

Our heterogeneous-speed results are complementary to existing studies on the welfare consequences of high-frequency trading (HFT). First, the existing theoretical literature on HFT typically assumes that fast traders also possess superior information about the value of the asset at the time of trading (see Biais, Foucault, and Moinas (2015), Budish, Cramton, and Shim (2015), Hoffmann (2014), Jovanovic and Menkveld (2012), and Cespa and Vives (2013)). By contrast, the fast trader in our model are not more informed speculators but act as rentextracting intermediaries. Second, the most commonly raised welfare question in the existing HFT literature is whether investment in high-speed trading technology is socially wasteful (see Biais, Foucault, and Moinas (2015), Pagnotta and Philippon (2013), Budish, Cramton, and Shim (2015), and Hoffmann (2014)). By contrast, our welfare question focuses on how trading frequency interacts with imperfect competition and the efficient allocations of assets, which is orthogonal to investments in speed technology. 


\section{Dynamic Trading in Sequential Double Auctions}

This section presents the dynamic trading model and characterizes the equilibrium and its properties. Main model parameters are tabulated in Appendix A for ease of reference.

\section{1 $\quad$ Model}

Timing and the double auctions mechanism. Time is continuous, $\tau \in[0, \infty)$. There are $n \geq 3$ risk-neutral traders in the market trading a divisible asset. Trading is organized as a sequence of uniform-price divisible double auctions, held at clock times $\{0, \Delta, 2 \Delta, 3 \Delta, \ldots\}$, where $\Delta>0$ is the length of clock time between consecutive auctions. The trading frequency of this market is therefore the number of double auctions per unit of clock time, i.e., $1 / \Delta$. The smaller is $\Delta$, the higher is the trading frequency. We will refer to the time interval $[t \Delta,(t+1) \Delta)$ as "period $t$," for $t \in\{0,1,2, \ldots\}$. Thus, the period- $t$ double auction occurs at the clock time $t \Delta$. The top plot of Figure 1 illustrates the timing of the double auctions.

Figure 1: Model time lines. The top plot shows times of double auctions, and the bottom plot shows the news times (dividend shocks, signals of dividend shocks, and private value shocks).

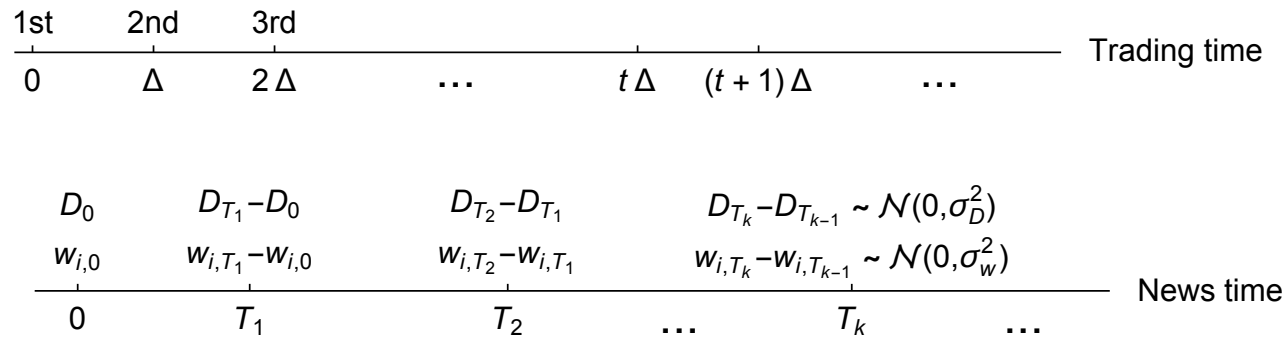

We denote by $z_{i, t \Delta}$ the inventory held by trader $i$ immediately before the period- $t$ double auction. The ex-ante inventories $z_{i, 0}$ are given exogenously. The total ex-ante inventory, $Z \equiv$ $\sum_{i} z_{i, 0}$, is common knowledge, and we assume that $Z$ does not change over time. (In securities markets, $Z$ can be interpreted as the total asset supply. In derivatives markets, $Z$ is by definition zero.) As shown later, while the equilibrium characterization works for any ex-ante inventory profile $\left\{z_{i, 0}\right\}_{i=1}^{n}$, in the analysis of trading frequency we will pay particular attention to the special case in which all traders are ex-ante identical (i.e. $z_{i, 0}=Z / n$ ).

A double auction is essentially a demand-schedule-submission game. In period $t$ each trader submits a demand schedule $x_{i, t \Delta}(p): \mathbb{R} \rightarrow \mathbb{R}$. The market-clearing price in period $t, p_{t \Delta}^{*}$, satisfies

$$
\sum_{i=1}^{n} x_{i, t \Delta}\left(p_{t \Delta}^{*}\right)=0
$$


In the eventual equilibrium we characterize, the demand schedules are strictly downward-sloping in $p$ and hence the solution $p_{t \Delta}^{*}$ exists and is unique. The evolution of inventory is given by

$$
z_{i,(t+1) \Delta}=z_{i, t \Delta}+x_{i, t \Delta}\left(p_{t \Delta}^{*}\right)
$$

After the period- $t$ double auction, each trader $i$ receives $x_{i, t \Delta}\left(p_{t \Delta}^{*}\right)$ units of the assets at the price of $p_{t \Delta}^{*}$ per unit. Of course, a negative $x_{i, t \Delta}\left(p_{t \Delta}^{*}\right)$ is a sale.

The asset. Each unit of the asset pays a single liquidating dividend $D$ at a random future time $\mathcal{T}$, where $\mathcal{T}$ follows an exponential distribution with parameter $r>0$, or mean $1 / r$. The random dividend time $\mathcal{T}$ is independent of all else in the model.

Before being paid, the dividend $D$ is unobservable and evolves as follows. At time $T_{0}=0$, $D=D_{0}$ is drawn from the normal distribution $\mathcal{N}\left(0, \sigma_{D}^{2}\right)$. Strictly after time 0 but conditional on the dividend time $\mathcal{T}$ having not arrived, the dividend $D$ is shocked at each of the clock times $T_{1}, T_{2}, T_{3}, \ldots$ The shock times $\left\{T_{k}\right\}_{k \geq 1}$ can be deterministic or stochastic. The dividend shocks at each $T_{k}$, for $k \geq 1$, are also i.i.d. normal with mean 0 and variance $\sigma_{D}^{2}$ :

$$
D_{T_{k}}-D_{T_{k-1}} \sim \mathcal{N}\left(0, \sigma_{D}^{2}\right)
$$

We will also refer to $\left\{T_{k}\right\}_{k \geq 0}$ as "news times." Thus, before the dividend is paid, the unobservable dividend $\left\{D_{\tau}\right\}_{\tau \geq 0}$ follows a jump process:

$$
D_{\tau}=D_{T_{k}}, \text { if } T_{k} \leq \tau<T_{k+1}
$$

Therefore, at the dividend payment time $\mathcal{T}$, the realized dividend is $D_{\mathcal{T}}$.

Since the expected dividend payment time is finite $(1 / r)$, for simplicity we normalize the discount rate to be zero (i.e., there is no time discounting). Allowing a positive time discounting does not change our qualitative results. Moreover, in the supplemental material to this paper, we provide an extension in which infinitely many dividends are paid sequentially and there is a time discount. The main results of this paper are robust to this extension.

Information and preference. At news time $T_{k}, k \in\{0,1,2, \cdots\}$, each trader $i$ receives a private signal $S_{i, T_{k}}$ about the dividend shock:

$$
S_{i, T_{k}}=D_{T_{k}}-D_{T_{k-1}}+\epsilon_{i, T_{k}}, \text { where } \epsilon_{i, T_{k}} \sim \mathcal{N}\left(0, \sigma_{\epsilon}^{2}\right) \text { are i.i.d., }
$$

and where $D_{T_{-1}} \equiv 0$. The private signals of trader $i$ are never disclosed to anyone else. If signals about dividend shocks were perfect, i.e. $\sigma_{\epsilon}^{2}=0$, the information structure of our model 
would be similar to that of Vayanos (1999).

In addition, each trader $i$ has a private value $w_{i, \mathcal{T}}$ for receiving the dividend, which can reflect tax or risk-management considerations. Formally, upon receiving the dividend $D_{\mathcal{T}}$, trader $i$ derives an additional benefit $w_{i, \mathcal{T}}$ per unit of the asset beyond the common dividend $D_{\mathcal{T}}$. The private values are also shocked at the news times $\left\{T_{k}\right\}_{k \geq 0}$, and each private-value shock is i.i.d. normal random variables with mean zero and variance $\sigma_{w}^{2}$ :

$$
w_{i, T_{k}}-w_{i, T_{k-1}} \sim \mathcal{N}\left(0, \sigma_{w}^{2}\right),
$$

where $w_{i, T_{-1}} \equiv 0$. Written in continuous time, trader $i$ 's private-value process $w_{i, \tau}$ is a jump process:

$$
w_{i, \tau}=w_{i, T_{k}} \text {, if } T_{k} \leq \tau<T_{k+1} .
$$

The private values to trader $i$ are observed by himself and are never disclosed to anyone else.

Therefore, if the dividend is paid at time $\tau$, trader $i$ receives

$$
v_{i, \tau} \equiv D_{\tau}+w_{i, \tau}
$$

per unit of asset held. ${ }^{4}$

The bottom plot of Figure 1 illustrates the news times $\left\{T_{k}\right\}_{k \geq 0}$, when dividend shocks, the signals of dividend shocks, and the private-value shocks all arrive. The two plots of Figure 1 make it clear that, in our model, the fundamental properties of the asset are separate from the trading frequency of the market.

Moreover, in an interval $[t \Delta,(t+1) \Delta)$ but before the dividend $D$ is paid, trader $i$ incurs a "flow cost" that is equal to $0.5 \lambda z_{i,(t+1) \Delta}^{2}$ per unit of clock time, where $\lambda>0$ is a commonly known constant. The quadratic flow cost is essentially a dynamic version of the quadratic cost used in the static models of Vives (2011) and Rostek and Weretka (2012). We can also interpret this flow cost as an inventory cost, which can come from regulatory capital requirements, collateral requirement, or risk-management considerations. (This inventory cost is not strictly risk aversion, however.) Once the dividend is paid, the flow cost no longer applies. Thus, conditional on the dividend having not been paid by time $t \Delta$, each trader suffers the flow cost for a duration of $\min (\Delta, \mathcal{T}-t \Delta)$ within period $t$, with the expectation

$$
\mathbb{E}[\min (\Delta, \mathcal{T}-t \Delta) \mid \mathcal{T}>t \Delta]=\int_{0}^{\infty} r e^{-r \tau} \min (\tau, \Delta) d \tau=\frac{1-e^{-r \Delta}}{r}
$$

\footnotetext{
${ }^{4}$ As in Wang (1994), the unconditional mean of the dividend here is zero, but one could add a positive constant to $D$ so that the probability of $D<0$ or $v<0$ is arbitrarily small. Moreover, in the markets for many financial and commodity derivatives - including forwards, futures and swaps - cash flows can become arbitrarily negative as market conditions change over time.
} 
where we have used the fact that, given the memoryless property of exponential distribution, $\mathcal{T}-t \Delta$ is exponentially distributed with mean $1 / r$ conditional on $\mathcal{T}>t \Delta$.

Value function and equilibrium definition. For conciseness of expressions, we let $H_{i, \tau}$ be the "history" (information set) of trader $i$ at time $\tau$ :

$$
H_{i, \tau}=\left\{\left\{\left(S_{i, T_{l}}, w_{i, T_{l}}\right)\right\}_{T_{l} \leq \tau},\left\{z_{t^{\prime} \Delta}\right\}_{t^{\prime} \Delta \leq \tau},\left\{x_{i, t^{\prime} \Delta}(p)\right\}_{t^{\prime} \Delta<\tau}\right\}
$$

That is, $H_{i, \tau}$ contains trader $i$ 's asset value-relevant information received up to time $\tau$, trader $i$ 's path of inventories up to time $\tau$, and trader $i$ 's demand schedules in double auctions before time $\tau$. Notice that by the identity $z_{i,\left(t^{\prime}+1\right) \Delta}-z_{i, t^{\prime} \Delta}=x_{i, t^{\prime} \Delta}\left(p_{t^{\prime} \Delta}^{*}\right)$, a trader can infer from $H_{i, \tau}$ the price in any past period $t^{\prime}$. Notice also that $H_{i, t \Delta}$ does not include the outcome of the period- $t$ double auction.

Let $V_{i, t \Delta}$ be trader $i$ 's period- $t$ continuation value immediately before the double auction at time $t \Delta$. By the definition of $H_{i, \tau}$, trader $i$ 's information set right before the period- $t$ double auction is $H_{i, t \Delta}$. We can write $V_{i, t \Delta}$ recursively as:

$$
\begin{aligned}
V_{i, t \Delta}=\mathbb{E}[ & -x_{i, t \Delta}^{*} p_{t \Delta}^{*}+\left(1-e^{-r \Delta}\right)\left(z_{i, t \Delta}+x_{i, t \Delta}^{*}\right) v_{i, t \Delta}+e^{-r \Delta} V_{i,(t+1) \Delta} \\
& \left.-\frac{1-e^{-r \Delta}}{r} \cdot \frac{\lambda}{2}\left(z_{i, t \Delta}+x_{i, t \Delta}^{*}\right)^{2} \mid H_{i, t \Delta}\right],
\end{aligned}
$$

where $x_{i, t \Delta}^{*}$ is a shorthand for $x_{i, t \Delta}\left(p_{t \Delta}^{*}\right)$. The first term $-x_{i, t \Delta}^{*} p_{t \Delta}^{*}$ is trader $i$ 's net cash flow for buying $x_{i, t \Delta}^{*}$ units at $p_{t \Delta}^{*}$ each. The second term $\left(1-e^{-r \Delta}\right)\left(z_{i, t \Delta}+x_{i, t \Delta}^{*}\right) v_{i, t \Delta}$ says that if the dividend is paid during period $t$, which happens with probability $1-e^{-r \Delta}$, then trader $i$ receives $\left(z_{i, t \Delta}+x_{i, t \Delta}^{*}\right) v_{i, t \Delta}$ in expectation. (Since shocks to the common dividend and private values have mean zero, trader $i$ 's expected value is still $v_{i, t \Delta}$ even if more news arrives during period t.) The third term $e^{-r \Delta} V_{i,(t+1) \Delta}$ says that if the dividend is not paid during period $t$, which happens with probability $e^{-r \Delta}$, trader $i$ receives the next-period continuation value $V_{i,(t+1) \Delta}$. Finally, the last term $-\frac{1-e^{-r \Delta}}{r} \cdot \frac{\lambda}{2}\left(z_{i, t \Delta}+x_{i, t \Delta}^{*}\right)^{2}$ is the expected quadratic inventory cost incurred during period $t$ for holding $z_{i, t \Delta}+x_{i, t \Delta}^{*}$ units of the asset (see Equation (9)). 
We can expand the recursive definition of $V_{i, t \Delta}$ explicitly:

$$
\begin{aligned}
V_{i, t \Delta}=\mathbb{E}[ & -\sum_{t^{\prime}=t}^{\infty} e^{-r\left(t^{\prime}-t\right) \Delta} x_{i, t^{\prime} \Delta}^{*} p_{t^{\prime} \Delta}^{*}+\sum_{t^{\prime}=t}^{\infty} e^{-r\left(t^{\prime}-t\right) \Delta}\left(1-e^{-r \Delta}\right) v_{i, t^{\prime} \Delta}\left(z_{i, t^{\prime} \Delta}+x_{i, t^{\prime} \Delta}^{*}\right) \\
& \left.-\frac{1-e^{-r \Delta}}{r} \sum_{t^{\prime}=t}^{\infty} e^{-r\left(t^{\prime}-t\right) \Delta} \frac{\lambda}{2}\left(z_{i, t^{\prime} \Delta}+x_{i, t^{\prime} \Delta}^{*}\right)^{2} \mid H_{i, t \Delta}\right] .
\end{aligned}
$$

While trader $i$ 's continuation value $V_{i, t \Delta}$ in principle can depend on anything in his information set $H_{i, t \Delta}$, in the eventual equilibrium we characterize, it will depend on trader $i$ 's current pre-auction inventory $z_{i, t \Delta}$, his current private value $w_{i, t \Delta}$, and the sum of his private signals $\sum_{l: T_{l} \leq t \Delta} S_{i, T_{l}}$ about the dividend.

Definition 1 (Perfect Bayesian Equilibrium). A perfect Bayesian equilibrium is a strategy profile $\left\{x_{j, t \Delta}\right\}_{1 \leq j \leq n, t \geq 0}$, where each $x_{i, t \Delta}$ depends only on $H_{i, t \Delta}$, such that for every trader $i$ and at every path of his information set $H_{i, t \Delta}$, trader $i$ has no incentive to deviate from $\left\{x_{i, t^{\prime} \Delta}\right\}_{t^{\prime} \geq t}$. That is, for every alternative strategy $\left\{\tilde{x}_{i, t^{\prime} \Delta}\right\}_{t^{\prime} \geq t}$, we have:

$$
V_{i, t \Delta}\left(\left\{x_{i, t^{\prime} \Delta}\right\}_{t^{\prime} \geq t},\left\{x_{j, t^{\prime} \Delta}\right\}_{j \neq i, t^{\prime} \geq t}\right) \geq V_{i, t \Delta}\left(\left\{\tilde{x}_{i, t^{\prime} \Delta}\right\}_{t^{\prime} \geq t},\left\{x_{j, t^{\prime} \Delta}\right\}_{j \neq i, t^{\prime} \geq t}\right)
$$

\subsection{The competitive benchmark equilibrium}

Before solving this model with imperfect competition and strategic trading, we first solve a competitive benchmark in which all traders take prices as given. In doing so, we will also solve the traders' inference of the dividend $D$ from equilibrium prices. The solution to this inference problem in the competitive equilibrium will be used directly in solving the strategic equilibrium later.

For clarity, we use the superscript " $c$ " to label the strategies, allocations, and prices in the competitive equilibrium. In each period $t$ each trader $i$ maximizes his continuation value $V_{i, t}$, defined in Equation (12), by choosing the optimal demand schedule $x_{i, t \Delta}^{c}\left(p_{t \Delta}^{c}\right)$, taking as given the period- $t$ price and the strategies of his own and other traders in subsequent periods.

We start by conjecturing that the competitive demand schedule $x_{i, t \Delta}^{c}\left(p_{t \Delta}^{c}\right)$ in period $t$ is such that trader $i$ 's expected marginal value for holding $z_{i, t \Delta}^{c}+x_{i, t \Delta}^{c}\left(p_{t \Delta}^{c}\right)$ units of the asset for the indefinite future is equal to the price $p_{t \Delta}^{c}$, for each $p_{t \Delta}^{c}$. That is, we conjecture that

$$
\mathbb{E}\left[v_{i, t \Delta} \mid H_{i, t \Delta}, p_{t \Delta}^{c}\right]-\frac{\lambda}{r}\left(z_{i, t \Delta}^{c}+x_{i, t \Delta}^{c}\left(p_{t \Delta}^{c}\right)\right)=p_{t \Delta}^{c}
$$

where the term $\lambda / r$ takes into account that the marginal holding cost is incurred for an expected 
duration of time $1 / r$. This conjecture can be rewritten as:

$$
x_{i, t \Delta}^{c}\left(p_{t \Delta}^{c}\right)=-z_{i, t \Delta}^{c}+\frac{r}{\lambda}\left(\mathbb{E}\left[v_{i, t \Delta} \mid H_{i, t \Delta}, p_{t \Delta}^{c}\right]-p_{t \Delta}^{c}\right) .
$$

The bulk of the remaining derivation involves finding an explicit expression for $\mathbb{E}\left[v_{i, t} \Delta\right.$ $\left.H_{i, t \Delta}, p_{t \Delta}^{c}\right]$. After that the optimal strategy is derived and verified.

Without loss of generality, let us focus on the period- $t$ double auction and suppose that the latest dividend shock is the $k$-th. Conditional on the unbiased signals $\left\{S_{j, T_{l}}\right\}_{0 \leq l \leq k}$ of dividend shocks, trader $j$ 's expected value of the dividend $D_{t \Delta}$ is a multiple of $\sum_{l=0}^{k} S_{j, T_{l}}$. Moreover, his private value, $w_{j, T_{k}}$, is perfectly observable to him. We thus conjecture that each trader $j$ uses the following symmetric linear strategy:

$$
x_{j, t \Delta}^{c}(p)=A_{1} \sum_{l=0}^{k} S_{j, T_{l}}+A_{2} w_{j, T_{k}}-\frac{r}{\lambda} p-z_{j, t \Delta}^{c}+f Z,
$$

where $A_{1}, A_{2}$, and $f$ are constants and where we have plugged in the coefficients of $p$ and $z_{j, t \Delta}$ from Equation (15). In particular, trader $j$ puts a weight of $A_{1}$ on his common-value information and a weight of $A_{2}$ on his private value.

By market clearing and the fact that $\sum_{j} z_{j, t \Delta}=Z$ is common knowledge, each trader $i$ is able to infer

$$
\sum_{j \neq i}\left(A_{1} \sum_{l=0}^{k} S_{j, T_{k}}+A_{2} w_{j, T_{k}}\right)
$$

from the equilibrium price $p_{t \Delta}^{c}$. Thus, each trader $i$ infers his value $v_{i, T_{k}} \equiv D_{T_{k}}+w_{i, T_{k}}$ by taking the conditional expectation:

$$
\begin{aligned}
& \mathbb{E}\left[v_{i, T_{k}} \mid H_{i, T_{k}}, \sum_{j \neq i}\left(A_{1} \sum_{l=0}^{k} S_{j, T_{l}}+A_{2} w_{j, T_{k}}\right)\right] \\
= & w_{i, T_{k}}+\mathbb{E}\left[D_{T_{k}} \mid \sum_{l=0}^{k} S_{i, T_{l}}, \sum_{j \neq i}\left(A_{1} \sum_{l=0}^{k} S_{j, T_{l}}+A_{2} w_{j, T_{k}}\right)\right] \\
= & w_{i, T_{k}}+B_{1} \sum_{l=0}^{k} S_{i, T_{l}}+B_{2} \underbrace{\sum_{j \neq i}\left(A_{1} \sum_{l=0}^{k} S_{j, T_{l}}+A_{2} w_{j, T_{k}}\right)}_{\text {Inferred from } p_{t \Delta}^{c}},
\end{aligned}
$$

where we have used the projection theorem for normal distributions and where the constants $B_{1}$ and $B_{2}$ are functions of $A_{1}, A_{2}$, and other primitive parameters. In particular, trader $i$ 's conditional expected value has a weight of $B_{1}$ on his common-value information $\sum_{l=0}^{k} S_{i, T_{l}}$ and 
a weight of 1 on his private value $w_{i, T_{k}}$. The third term is inferred from the price.

Because trader $i$ 's competitive strategy $x_{i, t \Delta}^{c}$ is linear in $\mathbb{E}\left[v_{i, T_{k}} \mid p_{t \Delta}^{*}, H_{i, T_{k}}\right]$, trader $i$ 's weight on his common-value information and his weight on the private value have a ratio of $B_{1}$. But by symmetric strategies, this ratio must be consistent with the conjectured strategy to start with, i.e., $B_{1}=A_{1} / A_{2}$. In Appendix C.1, we explicitly calculate that this symmetry pins down the ratio to be $B_{1}=A_{1} / A_{2} \equiv \chi$, where $\chi \in(0,1)$ is the unique solution to ${ }^{5}$

$$
\frac{1 /\left(\chi^{2} \sigma_{\epsilon}^{2}\right)}{1 /\left(\chi^{2} \sigma_{D}^{2}\right)+1 /\left(\chi^{2} \sigma_{\epsilon}^{2}\right)+(n-1) /\left(\chi^{2} \sigma_{\epsilon}^{2}+\sigma_{w}^{2}\right)}=\chi .
$$

On the left-hand side of Equation (19), we apply the projection theorem to Equation (18) to derive the weight $B_{1}$ as a function of $A_{1} / A_{2} \equiv \chi$. The projection theorem weighs the precision of the noise $\chi \epsilon_{i, T_{k}}$ in trader $i$ 's dividend signal, against the precision of the dividend shock $\chi\left(D_{T_{k}}-D_{T_{k-1}}\right)$ and the precision of others' dividend noise and private value $\sum_{j \neq i}\left(\chi \epsilon_{j, T_{k}}+w_{j, T_{k}}\right)$.

We define the "total signal" $s_{i, t \Delta}$ by

$$
\begin{aligned}
s_{i, T_{k}} & \equiv \frac{\chi}{\alpha} \sum_{l=0}^{k} S_{i, T_{l}}+\frac{1}{\alpha} w_{i, T_{k}}, \\
s_{i, \tau} & =s_{i, T_{k}}, \text { for } \tau \in\left[T_{k}, T_{k+1}\right),
\end{aligned}
$$

where the scaling factor $\alpha$ is defined to be

$$
\alpha \equiv \frac{\chi^{2} \sigma_{\epsilon}^{2}+\sigma_{w}^{2}}{n \chi^{2} \sigma_{\epsilon}^{2}+\sigma_{w}^{2}}>\frac{1}{n}
$$

Trader $i$ 's total signal incorporates the two-dimensional information $\left(\sum_{l=0}^{k} S_{i, T_{l}}, w_{i, T_{k}}\right)$ in a linear combination with weights $\chi / \alpha$ and $1 / \alpha$.

This construction of total signals leads to a very intuitive expression of the conditional expected value $v_{i, T_{k}}$. Direct calculation implies that (see details in Appendix C.1, Lemma 3)

$$
\mathbb{E}\left[v_{i, T_{k}} \mid H_{i, T_{k}}, \sum_{j \neq i} s_{j, T_{k}}\right]=\alpha s_{i, T_{k}}+\frac{1-\alpha}{n-1} \underbrace{\sum_{j \neq i} s_{j, T_{k}}}_{\text {Inferred from } p_{t \Delta}^{c}} .
$$

Equation (22) says that conditional on his own information and $\sum_{j \neq i} s_{j, T_{k}}$ (inferred from the equilibrium price), trader $i$ 's expected value of the asset is a weighted average of the total signals, with a weight of $\alpha>1 / n$ on his own total signal $s_{i, T_{k}}$ and a weight of $(1-\alpha) /(n-1)<1 / n$

\footnotetext{
${ }^{5}$ The left-hand side of Equation (19) is decreasing in $\chi$. It is $1 /\left(1+\sigma_{\epsilon}^{2} / \sigma_{D}^{2}\right)>0$ if $\chi=0$ and is $1 /(1+$ $\left.\sigma_{\epsilon}^{2} / \sigma_{D}^{2}+(n-1) /\left(1+\sigma_{w}^{2} / \sigma_{\epsilon}^{2}\right)\right)<1$ if $\chi=1$. Hence, Equation (19) has a unique solution $\chi \in \mathbb{R}$, and such solution satisfies $\chi \in(0,1)$.
} 
on each of the other traders' total signal $s_{j, T_{k}}$. The weights differ because other traders' total signals include both common dividend information and their private values, and others' private values are essentially "noise" to trader $i$ (hence under-weighting).

Substituting Equation (22) into Equation (15), we have

$$
x_{i, t \Delta}^{c}\left(p_{t \Delta}^{c}\right)=-z_{i, t \Delta}^{c}+\frac{r}{\lambda}\left(\alpha s_{i, t \Delta}+\frac{1-\alpha}{n-1} \sum_{j \neq i} s_{j, t \Delta}\right)-\frac{r}{\lambda} p_{t \Delta}^{c},
$$

By market clearing, $\sum_{i} x_{i, t \Delta}^{c}\left(p_{t \Delta}^{c}\right)=0$, we solve

$$
p_{t \Delta}^{c}=\frac{1}{n} \sum_{j=1}^{n} s_{j, t \Delta}-\frac{\lambda}{r n} Z
$$

The first term of $p_{t \Delta}^{c}$ is the average total signal, and the second term is the marginal cost of holding the average inventory $Z / n$ for an expected duration of time $1 / r$.

Substituting Equation (24) back to the expressions of $x_{i, t \Delta}^{c}\left(p_{t \Delta}^{c}\right)$ in Equation (23), we obtain explicitly the competitive demand schedule:

$$
x_{i, t \Delta}^{c}(p)=\frac{r(n \alpha-1)}{\lambda(n-1)}\left(s_{i, t \Delta}-p-\frac{\lambda(n-1)}{r(n \alpha-1)} z_{i, t \Delta}^{c}+\frac{\lambda(1-\alpha)}{r(n \alpha-1)} Z\right) .
$$

Appendix C.2 verifies that under this strategy the first-order condition of trader $i$ 's value function (12) can indeed be written in the form of Equation (15). The second-order condition is satisfied as $n \alpha>1$ by the definition of $\alpha$.

The post-trading allocation in the competitive equilibrium in period $t$ is:

$$
z_{i,(t+1) \Delta}^{c}=z_{i, t \Delta}^{c}+x_{i, t \Delta}^{c}\left(p_{t \Delta}^{c}\right)=\frac{r(n \alpha-1)}{\lambda(n-1)}\left(s_{i, t \Delta}-\frac{1}{n} \sum_{j=1}^{n} s_{j, t \Delta}\right)+\frac{1}{n} Z .
$$

That is, after each double auction, each trader is allocated the average inventory plus a constant multiple of how far his total signal deviates from the average total signal. We also see that the competitive inventories are martingales since total signals are martingales. We refer to this allocation as the "competitive allocation."

The following proposition summarizes the competitive equilibrium.

Proposition 1. In the competitive equilibrium, the strategies are given by Equation (25), the price by Equation (24), and the allocations by Equation (26). 


\subsection{Characterizing the strategic equilibrium}

Having solved a competitive benchmark, we now turn to the equilibrium with imperfect competition and strategic behavior, i.e., traders take into account the impact of their trades on prices. The equilibrium is stated in the following proposition.

Proposition 2. Suppose that $n \alpha>2$, which is equivalent to

$$
\frac{1}{n / 2+\sigma_{\epsilon}^{2} / \sigma_{D}^{2}}<\sqrt{\frac{n-2}{n}} \frac{\sigma_{w}}{\sigma_{\epsilon}}
$$

With strategic bidding, there exists a perfect Bayesian equilibrium in which every trader $i$ submits the demand schedule

$$
x_{i, t \Delta}\left(p ; s_{i, t \Delta}, z_{i, t \Delta}\right)=b\left(s_{i, t \Delta}-p-\frac{\lambda(n-1)}{r(n \alpha-1)} z_{i, t \Delta}+\frac{\lambda(1-\alpha)}{r(n \alpha-1)} Z\right),
$$

where

$$
b=\frac{(n \alpha-1) r}{2(n-1) e^{-r \Delta} \lambda}\left((n \alpha-1)\left(1-e^{-r \Delta}\right)+2 e^{-r \Delta}-\sqrt{(n \alpha-1)^{2}\left(1-e^{-r \Delta}\right)^{2}+4 e^{-r \Delta}}\right)>0 .
$$

The period-t equilibrium price is

$$
p_{t \Delta}^{*}=\frac{1}{n} \sum_{i=1}^{n} s_{i, t \Delta}-\frac{\lambda}{r n} Z
$$

The derivation of the strategic equilibrium follows similar steps to that of the competitive equilibrium derived in Section 2.2. The details of equilibrium construction are delegated to Appendix C.3. Below, we discuss key intuition of the strategic equilibrium by comparing it with the competitive one.

Let us start with common properties shared between the strategic equilibrium and the competitive one. For example, the equilibrium prices are equal under competitive and strategic bidding. Equal price implies equal information inference from the price in both equilibria, hence equal construction of the total signal $\left\{s_{i, t \Delta}\right\}$ that consolidates traders' information about the common dividend and private values. The price aggregates the most recent total signals $\left\{s_{i, t \Delta}\right\}$, which has a flavor of rational expectations equilibrium. Since the total signals are martingales, the price is also a martingale. The second term $-\lambda Z /(n r)$ in $p_{t \Delta}^{*}$ and $p_{t \Delta}^{c}$ is the expected marginal cost of holding the average inventory $Z / n$ until the dividend is paid, i.e., for an expected duration of time $1 / r$. Although each trader learns from $p_{t \Delta}^{*}$ the average total signal $\sum_{i} s_{i, t \Delta} / n$ in period $t$, he does not learn the total signal or inventory of any other individual 
trader. Nor does a trader perfectly distinguish the common-value component and the privatevalue component of the price. Thus, private information is not fully revealed after each round of trading. Finally, the equilibrium strategies in Equations (28) and (25) are stationary: a trader's strategy only depends on his most recent total signal $s_{i, t} \Delta$ and his current inventory $z_{i, t \Delta}$, but does not depend explicitly on $t .^{6}$

There are two important differences between the strategic equilibrium of Proposition 2 and the competitive benchmark in Section 2.2. First, in the strategic equilibrium, rather than take the price as given, each trader in each period effectively selects a price-quantity pair from the residual demand schedule of all other traders. To mitigate price impact, they trade less aggressively in the strategic equilibrium than in the competitive equilibrium. Formally, the endogenous coefficient $b$ in Equation (28) is strictly smaller than $\frac{r(n \alpha-1)}{\lambda(n-1)}$ in Equation (25):

$$
\frac{b}{\frac{r(n \alpha-1)}{\lambda(n-1)}}=1+\frac{(n \alpha-1)\left(1-e^{-r \Delta}\right)-\sqrt{(n \alpha-1)^{2}\left(1-e^{-r \Delta}\right)^{2}+4 e^{-r \Delta}}}{2 e^{-r \Delta}}<1 .
$$

This feature is the familiar "bid shading" or "demand reduction" in models of divisible auctions (see Ausubel, Cramton, Pycia, Rostek, and Weretka 2014). The coefficient $b$ captures how much additional quantity of the asset a trader is willing to buy if the price drops by one unit per period. Thus, a smaller $b$ corresponds to a less aggressive demand schedule. As the number $n$ of traders tends to infinity, the ratio in Equation (31) tends to 1, so the strategic equilibrium converges to the competitive equilibrium.

Intimately related to the aggressiveness of demand schedules is the extent to which a trader "liquidates" his inventory in each trading round. In the competitive equilibrium strategy $x_{i, t}^{c}$, the coefficient in front of $z_{i, t}^{c}$ is -1 , meaning that each trader liquidates his inventory entirely. By contrast, under the strategy $x_{i, t \Delta}$ of Proposition 2, the coefficient in front of $z_{i, t \Delta}$ is

$$
d \equiv-b \frac{\lambda(n-1)}{r(n \alpha-1)}=-1+\frac{\sqrt{(n \alpha-1)^{2}\left(1-e^{-r \Delta}\right)^{2}+4 e^{-r \Delta}}-(n \alpha-1)\left(1-e^{-r \Delta}\right)}{2 e^{-r \Delta}}
$$

which, under the condition $n \alpha>2$, is strictly between -1 and 0 . Thus, each trader only

\footnotetext{
${ }^{6}$ Some readers may wonder why our model does not have the infinite-regress problem of beliefs about beliefs, beliefs about beliefs about beliefs, etc. The reason is that the equilibrium price reveals the average total signal in each period; thus, a trader's belief about the common dividend, as well as his potential high-order beliefs, is actually spanned by this trader's own private information and the equilibrium price. This logic was previously used by He and Wang (1995) and Foster and Viswanathan (1996) to show that the potential infinite-regress problem is resolved in their dynamic models with heterogenous information. Our assumption that the common dividend and private values evolve as random walks implies that only the current price has the most updated information and hence allows us to characterize a stationary equilibrium. Without the random walk assumption, traders may potentially need to use all past prices to form inference, and the analysis will become much more complicated.
} 
liquidates a fraction $|d|<1$ of his inventory, leaving a fraction $1+d \in(0,1)$. Partial liquidation of inventory implies that the strategy in period $t$ has an impact on strategies in all future periods, and that an inefficient allocation in one period affects the inefficiency in all future periods. The next subsection investigates in details how the quantity $1+d$ determines allocative inefficiency, which is ultimately related to the optimal trading frequency that we study in Section 3.

Relative to the competitive benchmark, the second important difference of the strategic equilibrium is that its existence requires $n \alpha>2$, which, as we show in the proof, is equivalent to the condition (27). If and only if $n \alpha>2$ is the coefficient $b$ positive, i.e. demand is decreasing in price. Condition (27) essentially requires that adverse selection regarding the common dividend is not "too large" relative to the gains from trade over private values. All else equal, condition (27) holds if $n$ is sufficiently large, if signals of dividend shocks are sufficiently precise (i.e. $\sigma_{\epsilon}^{2}$ is small enough), if new information on the common dividend is not too volatile (i.e. $\sigma_{D}^{2}$ is small enough), or if shocks to private values are sufficiently volatile (i.e. $\sigma_{w}^{2}$ is large enough). ${ }^{7}$ All these conditions reduce adverse selection. The intuition is that if a trader observes a higher equilibrium price, he infers that other traders have either higher private values or more favorable information about the common dividend. If the trader attributes too much of the higher price to a higher dividend, he may end up buying more conditional on a higher price, which leads to a negative $b$ and violates the second order condition. Learning from prices does not cause such a problem in the competitive equilibrium because a higher price there also reflects traders' disregard of price impact. Thus, conditional on the same price, traders do not learn as much about the dividend in the competitive equilibrium as in the strategic one.

The condition $n \alpha>2$ means that a trader's expected asset value has a weight of at least $2 / n$ on his own total signal and a weight of at most $(1-2 / n) /(n-1)=(n-2) /[n(n-1)]<1 / n$ on each of other traders' total signals. ${ }^{8}$ The condition $n \alpha>2$ is trivially satisfied if $\alpha=1$, which applies if dividend information is public $\left(\sigma_{\epsilon}^{2}=0\right.$ and $\left.\sigma_{w}^{2}>0\right)$ or if traders have pure private values $\left(\sigma_{D}^{2}=0\right.$ and $\left.\sigma_{w}^{2}>0\right)$.

We close this subsection with a brief discussion of equilibrium uniqueness. Since news times and trading times are separate in our model, it could happen that no new information arrives during one or more periods. For example, if no new information arrives in the time interval $((t-1) \Delta, t \Delta]$, then the period- $t$ double auction will have the same price as the period- $(t-1)$ double auction, i.e., the period- $t$ double auction looks like a public-information game. Vayanos

\footnotetext{
${ }^{7}$ As a special case, if $\sigma_{\epsilon}^{2}=0$ and $\sigma_{w}^{2}>0$, we have public information about the dividends, which implies $\chi=1$ and $\alpha=1$ from Equation (19). If $\sigma_{D}^{2}=0$ and $\sigma_{w}^{2}>0$, we have the pure private value case, which implies $\chi=0$ and $\alpha=1$. Each trader $i$ 's equilibrium strategy in the pure private value case and in the public dividend information case have the same coefficients on $s_{i, t \Delta}, p, z_{i, t \Delta}$, and $Z$.

${ }^{8}$ The existence condition for our equilibrium is analogous to Kyle, Obizhaeva, and Wang (2014)'s equilibrium existence condition that each trader believes that his signal about the asset value is roughly twice as precise as others traders believe it to be.
} 
(1999) shows that public-information games admit a continuum of equilibria, and he uses a trembling-hand argument to select one of them.

Our approach to equilibrium selection is to impose stationarity, i.e., the coefficients in the linear strategy are the same across all periods. Going back to the example, if no new information arrives in $((t-1) \Delta, t \Delta]$, the stationarity-selected equilibrium in the period- $t$ double auction will be identical to one in which fresh news does arrive in $((t-1) \Delta, t \Delta]$ but the realizations of the dividend shock, the $n$ signals of dividend shocks, and the $n$ private-value shocks all turn out to be zero. The following proposition shows that the equilibrium of Proposition 2 is unique if strategies are restricted to be linear and stationary.

Proposition 3. The equilibrium from Proposition 2 is the unique perfect Bayesian equilibrium in the following class of strategies:

$$
x_{i, t \Delta}(p)=\sum_{T_{l} \leq t \Delta} a_{l} S_{i, T_{l}}+a_{w} w_{i, t \Delta}-b p+d z_{i, t \Delta}+f
$$

where $\left\{a_{l}\right\}_{l \geq 0}, a_{w}, b, d$ and $f$ are constants.

As the proof of Proposition 2 makes clear, each trader's optimal strategy belongs to class (33) if other traders also use strategies of the class (33). Therefore, Equation (33) is not a restriction on the traders' strategy space, but rather a restriction on the domain of equilibrium uniqueness. (We have not ruled out the existence of non-linear equilibrium.)

\subsection{Efficiency and comparative statics}

We now study the allocative efficiency (or inefficiency) in the equilibrium of Proposition 2. The results of this section lay the foundation for the study of optimal trading frequency in the next section.

Let us denote by $\left\{z_{i, t \Delta}^{*}\right\}$ the path of inventories obtained by the equilibrium strategy $x_{i, t \Delta}$ of Proposition 2. By the definition of $d$ in Equation (32), $z_{i, t \Delta}^{*}$ evolves according to:

$$
\begin{aligned}
z_{i,(t+1) \Delta}^{*} & =z_{i, t \Delta}^{*}+x_{i, t \Delta}\left(p_{t \Delta}^{*} ; s_{i, t \Delta}, z_{i, t \Delta}^{*}\right) \\
& =(1+d) z_{i, t \Delta}^{*}+b\left(s_{i, t \Delta}-\frac{1}{n} \sum_{j} s_{j, t \Delta}+\frac{\lambda(n-1)}{r(n \alpha-1)} \frac{Z}{n}\right) \\
& =b\left(s_{i, t \Delta}-\frac{1}{n} \sum_{j} s_{j, t \Delta}\right)+\frac{1}{n} Z+(1+d)\left(z_{i, t \Delta}^{*}-\frac{1}{n} Z\right) \\
& =(1+d) z_{i, t \Delta}^{*}-d z_{i,(t+1) \Delta}^{c},
\end{aligned}
$$


where in the second line we have substituted in the equilibrium strategy $x_{i, t \Delta}^{*}$ and the equilibrium price $p_{t \Delta}^{*}$, and in the last line we have substituted in Equation (26).

Comparing Equation (34) to Equation (26), we can see two differences. First, the posttrading allocation in the strategic equilibrium has an extra term $(1+d)\left(z_{i, t \Delta}^{*}-Z / n\right)$. Since $1+d \in(0,1)$, any inventory imbalance at the beginning of period $t$ partly carries over to the next period. As discussed in the previous subsection, this is a direct consequence of demand reduction caused by strategic bidding. Second, because inventories cannot be liquidated quickly due to strategic bidding, traders are more reluctant to acquire inventory. Therefore, the coefficient in front of $s_{i, t \Delta}-\sum_{j} s_{j, t \Delta} / n$ in the strategic allocation (34) is smaller than that in the competitive allocation (26). That is, strategic bidding makes post-trading asset allocations less sensitive to the dispersion of information (as measured by the total signals).

The above derivation directly leads to the exponential convergence to the competitive allocation over time, shown in the next proposition.

Proposition 4. Given any $0 \leq \underline{t} \leq \bar{t}$, if $s_{i, t \Delta}=s_{i, \underline{t} \Delta}$ for every $i$ and every $t \in\{\underline{t}, \underline{t}+1, \ldots, \bar{t}\}$, then the equilibrium inventories $z_{i, t}^{*}$ satisfy: for every $i$,

$$
z_{i,(t+1) \Delta}^{*}-z_{i,(t+1) \Delta}^{c}=(1+d)^{t+1-\underline{t}}\left(z_{i, \underline{t} \Delta}^{*}-z_{i, \underline{t}+1) \Delta}^{c}\right), \quad \forall t \in\{\underline{t}, \underline{t}+1, \ldots, \bar{t}\}
$$

where $d \in(-1,0)$ is given by Equation (32).

Moreover, $1+d$ is decreasing in $n, r, \sigma_{w}^{2}$, and $\Delta$ but increasing in $\sigma_{D}^{2}$. And $(1+d)^{1 / \Delta}$ is increasing in $\Delta$.

The convergence result of Proposition 4 is intuitive. If no new information arrives between period $\underline{t}$ and $\bar{t}$, then the competitive allocation remains unchanged, and Equation (35) follows from Equation (34) by induction.

Proposition 4 reveals that the strategic equilibrium is inefficient in allocating assets, although the allocative inefficiency converges to zero exponentially over time (as long as no new information arrives). After new dividend shocks and private-value shocks, the competitive allocation changes accordingly, and the strategic allocation starts to converge toward the new competitive allocation exponentially. Exponential convergence of this kind is previously obtained in the dynamic model of Vayanos (1999) under the assumption that common-value information is public.

The comparative statics of Proposition 4 are also intuitive, as illustrated by Figure 2. A smaller $1+d$ means faster convergence to efficiency. A larger $n$ makes traders more competitive, and a larger $r$ makes them more impatient. Both effects encourage aggressive bidding and speed up convergence. A large $\sigma_{D}^{2}$ implies a large uncertainty of a trader about the common asset value and a severe adverse selection; hence, in equilibrium the trader reduces his demand or supply 
Figure 2: Comparative statics of $1+d$. A smaller $1+d$ means faster convergence to the competitive allocation. Baseline parameters: $n=10, r=1 / 22, \lambda=1 / 1000, \sigma_{D}^{2}=1, \sigma_{e}^{2}=$ $1, \sigma_{w}^{2}=1 / 10$, and $\Delta=1 / 2$.
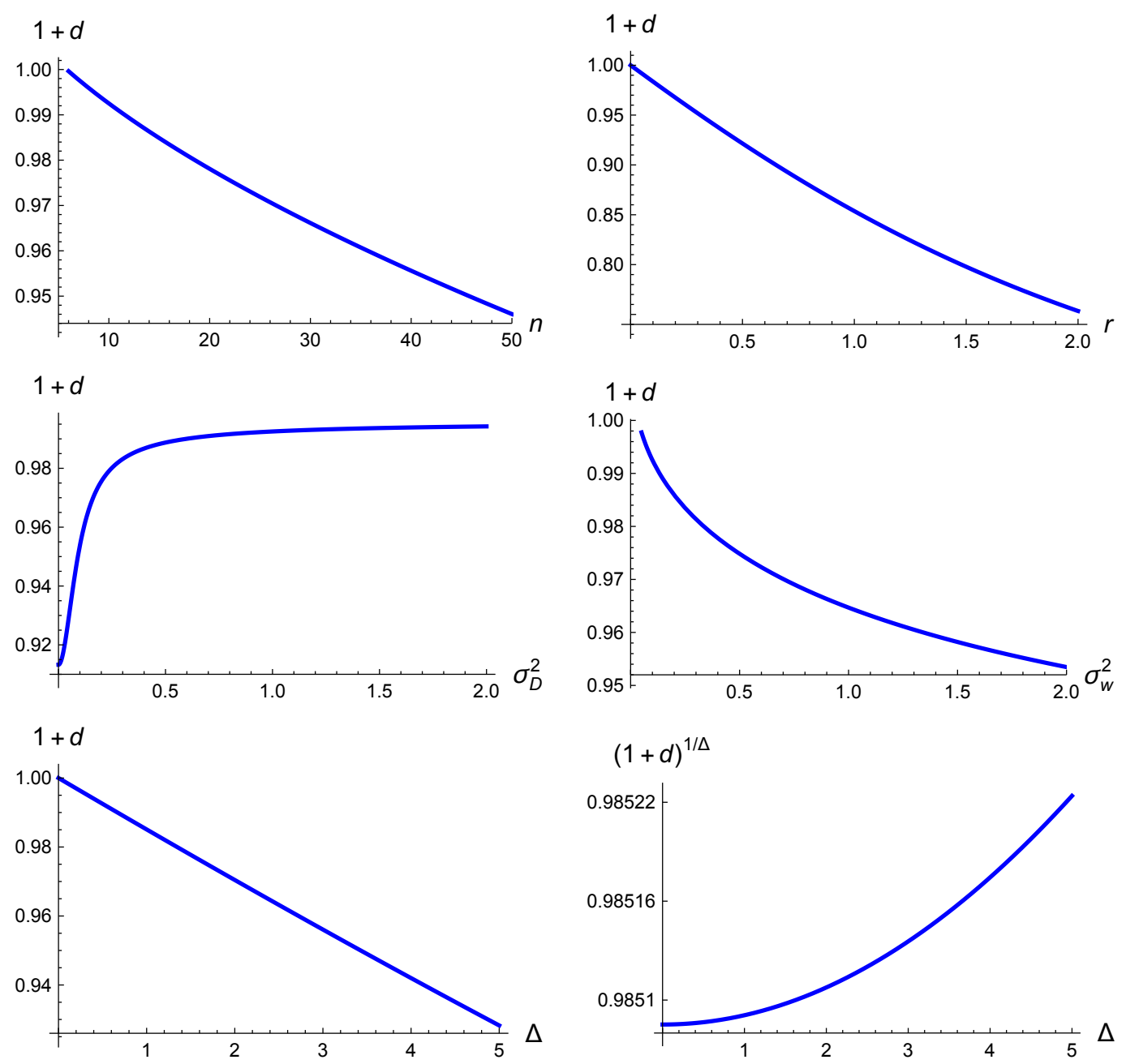

relative to the fully competitive market. Therefore, a higher $\sigma_{D}^{2}$ implies less aggressive bidding and slower convergence to the competitive allocation. The effect of $\sigma_{w}^{2}$ is the opposite: a higher $\sigma_{w}^{2}$ implies a larger gain from trade, and hence more aggressive bidding and faster convergence to the competitive allocation. The effects of $n, \sigma_{D}^{2}$ and $\sigma_{w}^{2}$ on bidding aggressiveness are present in earlier static models of Vives (2011) and Rostek and Weretka (2012). The effect of $\sigma_{D}^{2}$ in reducing the convergence speed to efficiency is also confirmed by Sannikov and Skrzypacz (2014) in a continuous-time trading model.

More novelly, $1+d$ is smaller if $\Delta$ is larger, that is, convergence per period is faster if trading frequency is lower. Intuitively, if traders have to wait for a long time before the next opportunity to trade, they have a strong incentive to trade aggressively in the current period. In this sense, a low-frequency market is a commitment device that counterbalances the strategic 
demand reduction. However, a higher trading frequency increases the convergence speed per unit of clock time (a lower $(1+d)^{1 / \Delta}$ ). This is because a higher number of trading opportunities more than offsets the lower aggressiveness per period. We caution that convergence speed is not the same as welfare, and the welfare implication of trading frequency is much subtler and richer, as we show in Section 3.

Finally, the comparative static of the speed of convergence with respect to $\sigma_{\epsilon}^{2}$ is ambiguous. It can be shown that the endogenous parameters $\alpha$ and $\chi$, and hence the speed of convergence, depend on the "normalized variances" $\sigma_{D}^{2} / \sigma_{\epsilon}^{2}$ and $\sigma_{w}^{2} / \sigma_{\epsilon}^{2}$. As $\sigma_{\epsilon}^{2}$ increases, $\sigma_{D}^{2} / \sigma_{\epsilon}^{2}$ and $\sigma_{w}^{2} / \sigma_{\epsilon}^{2}$ both decrease. A decrease in $\sigma_{D}^{2} / \sigma_{\epsilon}^{2}$ increases the speed of convergence, while a decrease in $\sigma_{w}^{2} / \sigma_{\epsilon}^{2}$ decreases the speed of convergence. The net effect is ambiguous.

\section{Welfare and Optimal Trading Frequency under Homo- geneous Trading Speed}

In this section and the next, we use the model framework developed in Section 2 to analyze the welfare implications of trading frequency. In this section we study the effect of trading frequency on welfare and characterize the optimal trading frequency if all traders participate in all double auctions, i.e., if traders have homogeneous trading speed. In the next section we study the case of heterogeneous trading speeds in the sense that fast traders participate in all double auctions but slow traders only participate periodically.

Throughout this section we conduct the analysis based on the perfect Bayesian equilibrium of Proposition 2, which requires the parameter condition $n \alpha>2$. Recall that $\left\{z_{i, t \Delta}^{*}\right\}$, defined by Equation (34), is the inventory path in the equilibrium of Proposition 2. We define the equilibrium welfare as the sum of the ex-ante expected utilities over all traders:

$$
W(\Delta)=\mathbb{E}\left[\sum_{i=1}^{n}\left(1-e^{-r \Delta}\right) \sum_{t=0}^{\infty} e^{-r t \Delta}\left(v_{i, t \Delta} z_{i,(t+1) \Delta}^{*}-\frac{\lambda}{2 r}\left(z_{i,(t+1) \Delta}^{*}\right)^{2}\right)\right]
$$

We denote the $\Delta$ that maximizes $W(\Delta)$ as $\Delta^{*}$. As usual, the price terms are canceled out as they are transfers. Note also that if all traders are ex-ante identical, with $z_{i, 0}=Z / n$, then each trader's ex-ante expected utility is simply $W(\Delta) / n$. Thus, the welfare criterion based on $W(\Delta)$ is equivalent to Parento dominance: If $W\left(\Delta_{1}\right)>W\left(\Delta_{2}\right)$ for $\Delta_{1} \neq \Delta_{2}$, then each trader $i$ 's ex-ante expected utility is higher under $\Delta_{1}$ than that under $\Delta_{2}$. 
Figure 3: Illustration of $z_{i, \tau}^{*}, z_{i, \tau}^{c}$, and $z_{i, \tau}^{e}$

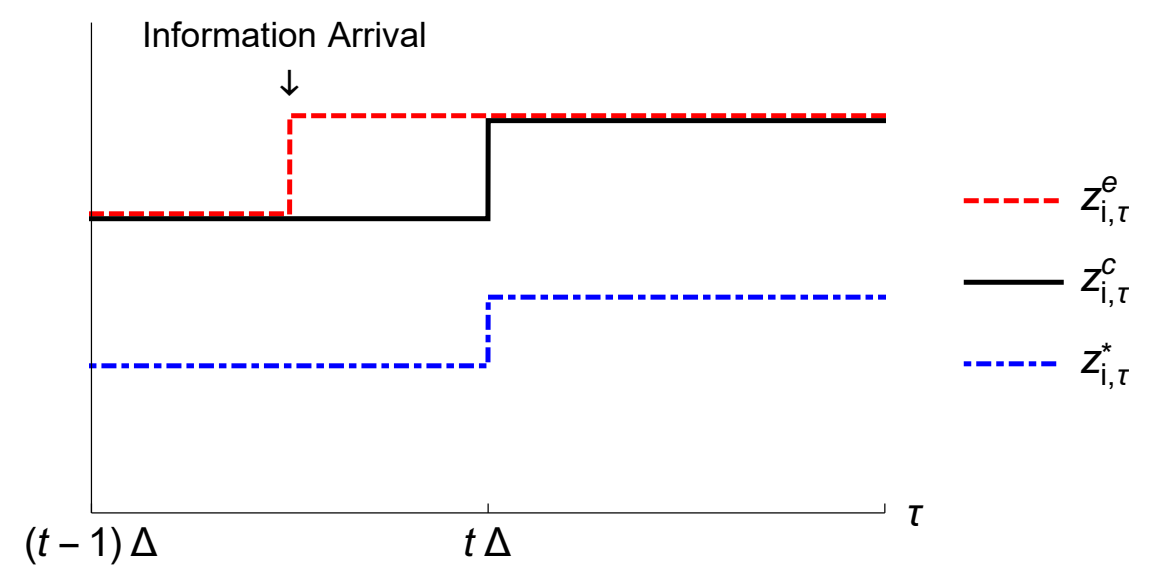

Analogously, we can define the welfare in the competitive equilibrium of Section 2.2 as:

$$
W^{c}(\Delta)=\mathbb{E}\left[\sum_{i=1}^{n}\left(1-e^{-r \Delta}\right) \sum_{t=0}^{\infty} e^{-r t \Delta}\left(v_{i, t \Delta} z_{i,(t+1) \Delta}^{c}-\frac{\lambda}{2 r}\left(z_{i,(t+1) \Delta}^{c}\right)^{2}\right)\right] .
$$

Although the competitive equilibrium is more efficient than the strategic one, it is still not fully efficient because new information may arrive between two double auctions. To explicitly take into account possible information arrivals, we define the continuous-time efficient allocation to be:

$$
z_{i, \tau}^{e}=\frac{r(n \alpha-1)}{\lambda(n-1)}\left(s_{i, \tau}-\frac{1}{n} \sum_{j=1}^{n} s_{j, \tau}\right)+\frac{1}{n} Z, \text { for every } \tau \geq 0 .
$$

The allocation $z_{i, \tau}^{e}$ is obtained in an idealized world in which a competitive double auction is held immediately after each news arrival. In constrast, at time $\tau \in(t \Delta,(t+1) \Delta]$, the strategic and competitive equilibrium allocations are, respectively, $z_{i, \tau}^{*}=z_{i,(t+1) \Delta}^{*}$ and $z_{i, \tau}^{c}=z_{i,(t+1) \Delta}^{c}$.

While $z_{i, \tau}^{e}$ and $z_{i,(t+1) \Delta}^{c}$ (defined in Equation (26)) have the same functional form (in particular, we have $\left.z_{i, t \Delta}^{e}=z_{i,(t+1) \Delta}^{c}\right)$, the difference is that $\left\{z_{i, \tau}^{e}\right\}$ changes at news time, whereas $\left\{z_{i, \tau}^{c}\right\}$ changes at trading time. Figure 3 provides an illustration. In this example information arrives strictly between period- $(t-1)$ and period- $t$ double auctions. We see that $z_{i, \tau}^{*}$ and $z_{i, \tau}^{c}$ only change at trading times, but $z_{i, \tau}^{e}$ changes immediately after news arrivals. The gap between $z_{i, \tau}^{*}$ and $z_{i, \tau}^{c}$ represents the inefficiency caused by strategic behavior, and the gap between $z_{i, \tau}^{c}$ and $z_{i, \tau}^{e}$ represents the inefficiency caused by the misalignment between trading times and news times. 
The ex-ante welfare under the efficient allocation is:

$$
W^{e}=\mathbb{E}\left[\sum_{i=1}^{n} \int_{\tau=0}^{\infty} r e^{-r \tau}\left(v_{i, \tau} z_{i, \tau}^{e}-\frac{\lambda}{2 r}\left(z_{i, \tau}^{e}\right)^{2}\right) d \tau\right]
$$

which is independent of $\Delta$.

With these primitives, the allocative inefficiency of the competitive equilibrium of Section 2.2 can be written as:

$$
X(\Delta) \equiv W^{e}-W(\Delta)=\underbrace{\left[W^{c}(\Delta)-W(\Delta)\right]}_{X_{1}(\Delta), \text { welfare cost of strategic behavior }}+\underbrace{\left[W^{e}-W^{c}(\Delta)\right]}_{X_{2}(\Delta), \text { welfare cost of trading delay }}
$$

The above equation decomposes the allocative inefficiency of the strategic equilibrium into two parts. The first part, call it $X_{1}(\Delta)$, is due to strategic behavior and demand reduction. The second part, call it $X_{2}(\Delta)$, is due to the potential misalignment between trading times and news times.

This decomposition highlights the important tradeoff in increasing trading frequency:

- A smaller $\Delta$ allows investors to react quickly to new information, reducing $X_{2}(\Delta)$.

- A smaller $\Delta$ also reduces the aggressiveness of demand schedules in each double auction, increasing $X_{1}(\Delta)$. This channel is a consequence of the fact that $(1+d)$ decreases in $\Delta$ (see Proposition 4).

We show in the two subsections that this tradeoff, and hence the optimal trading frequency, depend critically on the nature of new information (i.e., the shocks to dividends and private values). If new information arrives at deterministic and scheduled intervals, then trading times can be arranged such that $X_{2}(\Delta)=0$, and we show that the optimal trading frequency cannot be higher than the information arrival frequency. In the most natural case where all traders are ex-ante identical, the optimal trading frequency is equal to the information arrival frequency. By contrast, if new information arrives stochastically according to a Poisson process, then both $X_{1}(\Delta)$ and $X_{2}(\Delta)$ are generally positive, and this tradeoff leads to an interior optimal $\Delta^{*}$. We show that the optimal trading frequency $1 / \Delta^{*}$ is always higher than two-thirds of the information arrival frequency, and we provide conditions on $n \alpha$ under which $1 / \Delta^{*}$ can be arbitrarily larger than the information arrival frequency. We further provide comparative statics of $\Delta^{*}$ with respect to the asset characteristics. 


\subsection{Scheduled Arrivals of New Information}

We first consider scheduled information arrivals. In particular, we suppose that shocks to the common dividend and shocks to private values occur at regularly spaced clock times $T_{k}=k \gamma$ for a positive constant $\gamma$, where $k \geq 0$ are integers. Examples of scheduled information arrivals include macroeconomic data releases and corporate earnings announcements.

Proposition 5. Suppose $T_{k}=k \gamma$ for a positive constant $\gamma$. Then $W(\Delta)<W(\gamma)$ for any $\Delta<\gamma$. That is, $\Delta^{*} \geq \gamma$.

Proposition 5 shows that if new information repeatedly arrives at scheduled times, then the optimal trading frequency cannot be higher than the frequency of information arrivals.

For ease of exposition, we discuss here the intuition and the proof for the special case that $\gamma / \Delta$ is a positive integer. In this case, whenever scheduled information arrives, there is a double auction immediately following it. Since news times are entirely covered by trading times, the $X_{2}(\Delta)$ term in the decomposition (40) is zero. Thus, allocative inefficiency is entirely determined by welfare loss caused by strategic behavior, i.e. $X_{1}(\Delta)$. Below, we outline the main steps of proving that $X_{1}(\Delta)$ is decreasing in $\Delta$ if $\gamma / \Delta$ is a positive integer. Lemma 1 and Lemma 2 that we use below, however, hold for any information arrival process.

The following lemma relates the welfare loss of an allocation to the square distance between that allocation and the competitive allocation.

Lemma 1. For any profile of inventories $\left(z_{1}, z_{2}, \ldots, z_{n}\right)$ satisfying $\sum_{i=1}^{n} z_{i}=Z$ and any profile of total signals $\left(s_{1, t \Delta}, s_{2, t \Delta}, \ldots, s_{n, t \Delta}\right)$, we have:

$$
\begin{aligned}
& \sum_{i=1}^{n}\left(\left(\alpha s_{i, t \Delta}+\frac{1-\alpha}{n-1} \sum_{j \neq i} s_{j, t \Delta}\right) z_{i,(t+1) \Delta}^{c}-\frac{\lambda}{2 r}\left(z_{i,(t+1) \Delta}^{c}\right)^{2}\right) \\
& -\sum_{i=1}^{n}\left(\left(\alpha s_{i, t \Delta}+\frac{1-\alpha}{n-1} \sum_{j \neq i} s_{j, t \Delta}\right) z_{i}-\frac{\lambda}{2 r}\left(z_{i}\right)^{2}\right) \\
= & \frac{\lambda}{2 r} \sum_{i=1}^{n}\left(z_{i,(t+1) \Delta}^{c}-z_{i}\right)^{2} .
\end{aligned}
$$

Lemma 1 follows from the fact that each trader $i$ 's expected marginal value given the competitive allocation $z_{i,(t+1) \Delta}^{c}$ is exactly the competitive equilibrium price $p_{t \Delta}^{c}$ (see Appendix C.6 for details). Applied to the equilibrium allocations $x_{i, t \Delta}^{*}$, Lemma 1 implies that:

$$
X_{1}(\Delta)=\left(1-e^{-r \Delta}\right) \cdot \frac{\lambda}{2 r} \sum_{i=1}^{n} \sum_{t=0}^{\infty} e^{-r t \Delta} \mathbb{E}\left[\left(z_{i,(t+1) \Delta}^{*}-z_{i,(t+1) \Delta}^{c}\right)^{2}\right]
$$


An inspection of Equation (42) reveals that the effect of $\Delta$ on $X_{1}(\Delta)$ is not obvious. Proposition 4 shows that $1+d$ is decreasing in $\Delta$, but $(1+d)^{1 / \Delta}$ is increasing in $\Delta$. Thus, for each news arrival time $k \gamma$ and each trading time $t \Delta \in[k \gamma,(k+1) \gamma)$, as $\Delta$ increases, $\mathbb{E}\left[\left(z_{i,(t+1) \Delta}^{*}-z_{i,(t+1) \Delta}^{c}\right)^{2}\right]$ becomes smaller for a small $t \Delta-k \gamma$ but larger for a large $t \Delta-k \gamma$. This means that a higher trading frequency (a smaller $\Delta$ ) makes allocations less efficient in the early periods after a news arrival but more efficient in the later periods. Intuitively, a large $\Delta$ commits traders to submitting aggressive demand schedules whenever they can trade, leading to an immediate inventory reallocation early on. But a smaller $\Delta$ allows more "smoothing" of trades that eventually makes allocation more efficient if trading continues for a sufficiently long time. Adding to this tradeoff is the "discount factor" $e^{-r t \Delta}$, which gives more weight to relative allocative efficiency in early periods than in later periods in the assessment of welfare. Overall, the effect of $\Delta$ on $X_{1}(\Delta)$ seems ambiguous ex-ante. Showing the economic intuition of Proposition 5, therefore, boils down to showing why the (weighted) allocative efficiency in early periods dominates that in late periods.

This intricate tradeoff is illustrated in the left-hand panel of Figure 4, where we consider the special case of $\gamma=\infty$ (i.e., information arrives only once, at time 0 ) and plot the distance to efficient allocation, $\left|z_{i,(t+1) \Delta}^{*}-z_{i,(t+1) \Delta}^{c}\right|$, for two extreme trading frequencies: $\Delta=\infty$ (trading only once, at time 0$)$ and $\Delta=0$ (continuous trading). We can see that slower trading $(\Delta=\infty)$ leads to more efficient allocations in early periods, but faster trading $(\Delta=0)$ leads to more efficient allocations in late periods.

To proceed with the proof, we further simplify the square difference between the strategic and competitive equilibrium allocation, for $t \geq 1$ :

$$
\begin{aligned}
\mathbb{E}\left[\left(z_{i,(t+1) \Delta}^{*}-z_{i,(t+1) \Delta}^{c}\right)^{2}\right] & =(1+d)^{2} \mathbb{E}\left[\left(z_{i, t \Delta}^{*}-z_{i,(t+1) \Delta}^{c}\right)^{2}\right] \\
& =(1+d)^{2} \mathbb{E}\left[\left(z_{i, t \Delta}^{*}-z_{i, t \Delta}^{c}\right)^{2}\right]+(1+d)^{2} \mathbb{E}\left[\left(z_{i,(t+1) \Delta}^{c}-z_{i, t \Delta}^{c}\right)^{2}\right],
\end{aligned}
$$

where the first equality follows from Proposition 4, and the second equality follows from the fact that $z_{i, t}^{*}$ and $z_{i, t \Delta}^{c}$ are measurable with respect to the information in period $t-1$, and that $\left\{z_{i, t \Delta}^{c}\right\}_{t \geq 0}$ is a martingale, so $\mathbb{E}\left[\left(z_{i, t \Delta}^{*}-z_{i, t \Delta}^{c}\right)\left(z_{i,(t+1) \Delta}^{c}-z_{i, t \Delta}^{c}\right)\right]=0$ by the law of iterated expectations. Then by induction, we have:

$\mathbb{E}\left[\left(z_{i,(t+1) \Delta}^{*}-z_{i,(t+1) \Delta}^{c}\right)^{2}\right]=(1+d)^{2(t+1)} \mathbb{E}\left[\left(z_{i, 0}-z_{i, \Delta}^{c}\right)^{2}\right]+\sum_{t^{\prime}=1}^{t}(1+d)^{2\left(t-t^{\prime}+1\right)} \mathbb{E}\left[\left(z_{i,\left(t^{\prime}+1\right) \Delta}^{c}-z_{i, t^{\prime} \Delta}^{c}\right)^{2}\right]$

The above equation says that after auction $t$, allocative inefficiency is a linear combination of the inefficiency in initial allocations and the time variations in the competitive allocation up to time $t \Delta$. Substituting Equation (44) into the expression of $X_{1}$ in Equation (42), we get (see 
Appendix C.6 for details):

\section{Lemma 2.}

$$
X_{1}(\Delta)=\frac{\lambda(1+d)}{2 r(n \alpha-1)}\left(\sum_{i=1}^{n} \mathbb{E}\left[\left(z_{i, 0}-z_{i, \Delta}^{c}\right)^{2}\right]+\sum_{t=1}^{\infty} e^{-r t \Delta} \sum_{i=1}^{n} \mathbb{E}\left[\left(z_{i,(t+1) \Delta}^{c}-z_{i, t \Delta}^{c}\right)^{2}\right]\right) .
$$

The expression of $X_{1}(\Delta)$ in Equation (45) has an intuitive explanation. The first term in the big bracket, $\sum_{i=1}^{n} \mathbb{E}\left[\left(z_{i, 0}-z_{i, \Delta}^{c}\right)^{2}\right]$, measures the inefficiency of the initial allocation after time-0 information arrival. In the summation, each term $e^{-r t \Delta} \sum_{i=1}^{n} \mathbb{E}\left[\left(z_{i,(t+1) \Delta}^{c}-z_{i, t \Delta}^{c}\right)^{2}\right]$ measures how much the competitive allocation changes at each trading time, weighted by the probability that the dividend is not yet paid. Together, the terms in the big bracket represent the amount of allocative inefficiency measured at trading times that can potentially be eliminated. The leading multiplier, $\frac{\lambda(1+d)}{2 r(n \alpha-1)}$, says that because of strategic behavior, a fraction of the allocative inefficiency remains in the market. This fraction is lower if per-round convergence is faster, i.e., if $1+d$ is smaller.

To be even more explicit, let us now return to the special case that $\gamma / \Delta$ is a positive integer. In this case, news times are entirely covered by trading times, and

$$
z_{i,(t+1) \Delta}^{c}-z_{i, t \Delta}^{c} \begin{cases}\neq 0, & \text { if } t \Delta \text { is a multiple of } \gamma \\ =0, & \text { otherwise }\end{cases}
$$

where we have used the fact that the competitive allocations do not change without news arrivals. Dropping the zero terms, we can thus write the second term on the right-hand side of Equation (45) in terms of news time $\{k \gamma\}$ :

$$
\sum_{i=1}^{n} \sum_{k=1}^{\infty} e^{-r k \gamma} \mathbb{E}\left[\left(z_{i,(k+1) \gamma}^{c}-z_{i, k \gamma}^{c}\right)^{2}\right]
$$

where we have substituted in $z_{i, k \gamma+\Delta}^{c}=z_{i,(k+1) \gamma}^{c}$. Thus, the second term on the right-hand side of Equation (45) is purely a function of the news arrival process, and not a function of $\Delta$. The first term on the right-hand side of Equation (45) is purely a function of the news arriving at time 0 , again not a function of $\Delta$. Because $1+d$ is decreasing with $\Delta$ (Proposition 4 ), $X_{1}(\Delta)$ is decreasing in $\Delta$, which proves Proposition 5 for the cases that $\gamma / \Delta$ is a positive integer.

The right-hand panel of Figure 4 illustrates this net effect by plotting $W(\Delta)$ against $\Delta$, for the special case that $\gamma=\infty$. We can see that in this case $W(\Delta)$ increases in $\Delta$.

The same logic above applies to a general $\Delta \leq \gamma$, with some adjustment to the details. The proof of the general case of Proposition 5 is delegated to Appendix C.6.1. 
Figure 4: Trading frequency and welfare when information arrives only at time 0
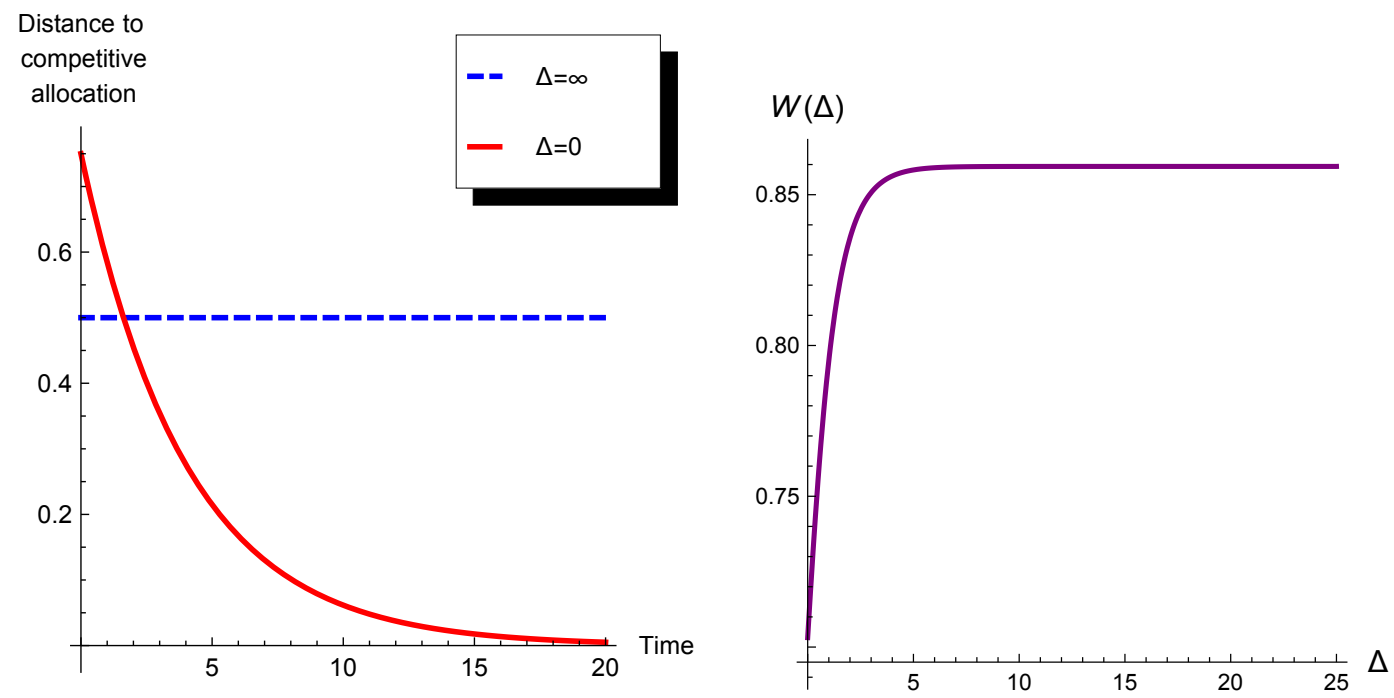

Proposition 5 establishes that the optimal trading frequency cannot be strictly higher than information frequency. Next, we ask if $\Delta^{*}$ can be strictly higher than $\gamma$. As $\Delta$ increases beyond $\gamma, X_{2}(\Delta)$ is generally positive. Thus, traders face the basic tradeoff we discussed at the beginning of this section: a large $\Delta>\gamma$ induces more aggressive trading per period, but incurs the cost that traders cannot react quickly to new information.

To further evaluate this tradeoff, we must first define some parameters that quantify the magnitude of the new information given by the dividend shocks. We define:

$$
\begin{aligned}
\sigma_{z}^{2} & \equiv \sum_{i=1}^{n} \mathbb{E}\left[\left(z_{i, T_{k}}^{e}-z_{i, T_{k-1}}^{e}\right)^{2}\right]=\left(\frac{r(n \alpha-1)}{\lambda(n-1)}\right)^{2} \frac{(n-1)\left(\chi^{2}\left(\sigma_{D}^{2}+\sigma_{\epsilon}^{2}\right)+\sigma_{w}^{2}\right)}{\alpha^{2}}>0 \\
\sigma_{0}^{2} & \equiv \sum_{i=1}^{n} \mathbb{E}\left[\left(z_{i, 0}-z_{i, 0}^{e}\right)^{2}\right]=\sum_{i=1}^{n} \mathbb{E}\left[\left(z_{i, 0}-z_{i, \Delta}^{c}\right)^{2}\right] .
\end{aligned}
$$

The first variance $\sigma_{z}^{2}$ describes the extent to which each arrival of new information changes the efficient inventories among traders. The second variance $\sigma_{0}^{2}$ describes the distance between the ex-ante inventory and the efficient inventory given the new information that arrives at time 0 . If $z_{i, 0}=Z / n$ for every trader $i$ (all traders are ex-ante identical), then $\sigma_{0}^{2}=\sigma_{z}^{2} \cdot{ }^{9}$ One may naturally view "time 0 " as a reduced-form representation of a steady state, in which case $\sigma_{0}^{2}$ and $\sigma_{z}^{2}$ should be equal. In the results below we will keep $\sigma_{0}^{2}$ as a generic parameter but highlight results for the most natural case of $\sigma_{0}^{2}=\sigma_{z}^{2}$.

\footnotetext{
${ }^{9}$ To see this, note that $Z / n$ is the efficient allocation to each trader if the initial total signals of all traders are zero. For each trader $i$, the total signal $s_{i, 0}$ received at time 0 has the same distribution as the innovation $s_{i, T_{k}}-s_{i, T_{k-1}}$. Thus, $z_{i, 0}^{e}-Z / n$ has the same distribution as $z_{i, T_{k}}^{e}-z_{i, T_{k-1}}^{e}$ for any $k \geq 1$. Thus, $\sigma_{0}^{2}=\sigma_{z}^{2}$ if $z_{i, 0}=Z / n$ for all $i$.
} 
The welfare $W(\Delta)$ is hard to analyze if $\Delta / \gamma$ is not an integer. For analytical tractability but at no cost of economic intuition, for the case of $\Delta>\gamma$ we restrict attention to $\Delta=l \gamma$ for a positive integer $l$. Let $l^{*} \in \operatorname{argmax}_{l \in \mathbb{Z}_{+}} W(l \gamma)$.

Proposition 6. Suppose that $T_{k}=k \gamma$ for a positive constant $\gamma$. The following results hold.

1. If $z_{i, 0}=Z / n$ for every trader $i$ (i.e., $\sigma_{0}^{2}=\sigma_{z}^{2}$ ), then $l^{*}=1$.

2. If $\sigma_{0}^{2} / \sigma_{z}^{2}$ remains bounded as $n \rightarrow \infty$, then $l^{*}=1$ as $n \rightarrow \infty$.

Part 1 of Proposition 6 states a sharp result: for the steady-state specification $\sigma_{0}^{2}=\sigma_{z}^{2}$, the optimal trading frequency is equal to the information frequency. To see the intuition, consider, for instance, slowing down trading from $\Delta=\gamma$ to $\Delta=2 \gamma$. Reducing the trading frequency by a half will make demand schedules more aggressive at time $0,2 \gamma, 4 \gamma, \ldots$, at the cost of entirely disabling reaction to new information at times $\gamma, 3 \gamma, 5 \gamma, \ldots$ But because new information at each arrival time is equally informative and in expectation shocks the efficient allocation by the same magnitude, there is no reason to let traders trade very aggressively over half of the news but shut down trading for the other half. Instead, it is better to allow equal opportunities respond to all information arrivals. That is, $l^{*}=1$. This intuition applies to any generic $\Delta=l \gamma$ for an integer $l>1$. The proof of Proposition 6 makes this intuitive argument formal.

Part 2 of Proposition 6 allows the time-0 information to be different from information arriving later. A sufficient condition for $\sigma_{0}^{2} / \sigma_{z}^{2}$ remaining bounded as $n \rightarrow \infty$ is $\mathbb{E}\left[\sum_{i=1}^{n}\left(z_{i, 0}-\right.\right.$ $\left.Z / n)^{2}\right]=O(n)$. If $\sigma_{0}^{2}>\sigma_{z}^{2}$, for instance, it is possible that the optimal $l^{*}>1$ so that eliminating the time-0 allocative inefficiency is more important than allowing immediate reaction to less important news later. That said, in a large market it is still asymptotically optimal to align trading times with information arrival time. The intuition is that as $n$ increases sufficiently, the market becomes almost competitive, and the inefficiency associated with strategic demand reduction diminishes. In the limit $n \rightarrow \infty, X_{1}(\Delta) \rightarrow 0$, and the allocation efficiency is entirely determined by how fast traders can react to new information. Thus, the optimal $l^{*}=1$.

\subsection{Stochastic Arrivals of New Information}

We now turn to stochastic arrivals of information. Examples of stochastic news include unexpected corporate announcements (e.g. mergers and acquisitions), regulatory actions, and geopolitical events. There are many possible specifications for stochastic information arrivals, and it is technically hard to calculate the optimal trading frequency for all of them. Instead, we analyze the simple yet natural case of a Poisson process for news arrivals. We expect the economic intuition of the results to apply to more general signal structures. 
Suppose that the timing of the news shocks $\left\{T_{k}\right\}_{k \geq 1}$ follows a homogeneous Poisson process with intensity $\mu>0$. (The first shock still arrives at time $T_{0}=0$.) Since the time interval between two consecutive news shocks has the expectation $1 / \mu, \mu$ is analogous to $1 / \gamma$ from Section 3.1. There are in expectation $\Delta \mu$ arrivals of new information during an interval of length $\Delta$, and each arrival of information shocks the squared difference in efficient allocation by $\sigma_{z}^{2}$ (see Equation (46)). Thus,

$$
\begin{aligned}
\sum_{i=1}^{n} \mathbb{E}\left[\left(z_{i,(t+1) \Delta}^{e}-z_{i, t \Delta}^{e}\right)^{2}\right] & =\Delta \mu \sigma_{z}^{2}, \\
\sum_{i=1}^{n} \mathbb{E}\left[\left(z_{i, \tau}^{e}-z_{i, t \Delta}^{e}\right)^{2}\right] & =(\tau-t \Delta) \mu \sigma_{z}^{2}, \quad \tau \in[t \Delta,(t+1) \Delta) .
\end{aligned}
$$

To gain further intuition, we now focus on the natural case that all traders are ex-ante identical (i.e. $\sigma_{0}^{2}=\sigma_{z}^{2}$ ) and explicitly spell out $X_{1}(\Delta)$ and $X_{2}(\Delta)$ from the decomposition $(40)$ :

$$
\begin{aligned}
& X_{1}(\Delta)=\frac{\lambda(1+d)}{2 r(n \alpha-1)}\left(1+\frac{\Delta e^{-r \Delta}}{1-e^{-r \Delta}} \mu\right) \sigma_{z}^{2}, \\
& X_{2}(\Delta)=\frac{\lambda}{2 r} \mathbb{E}\left[\int_{\tau=0}^{\infty} r e^{-r \tau}\left(z_{i, \tau}^{c}-z_{i, \tau}^{e}\right)^{2} d \tau\right]=\frac{\lambda}{2 r}\left(\frac{1}{r}-\frac{\Delta e^{-r \Delta}}{1-e^{-r \Delta}}\right) \mu \sigma_{z}^{2} .
\end{aligned}
$$

Here, the expression $X_{1}(\Delta)$ is obtained by substituting Equation (48) into Equation (45) from Lemma 2 (which applies to any information arrival process). We have also applied Lemma 1 to write $X_{2}(\Delta)$ as the expected square difference between $z_{i, \tau}^{c}$ and $z_{i, \tau}^{e}$. In fact, the final expression of $X_{2}(\Delta)$ can be rewritten as the more intuitive form:

$$
X_{2}(\Delta)=\frac{\lambda}{2} \cdot \underbrace{\left(\int_{0}^{\infty} e^{-r \tau} d \tau-\sum_{t=1}^{\infty} e^{-r t \Delta} \Delta\right)}_{\text {Misalignment of information arrival times and trading times }} \cdot \frac{\mu \sigma_{z}^{2}}{r} .
$$

The first term $\lambda / 2$ is the multiplier of quadratic holding cost. The middle term in the bracket represents the misalignment of information arrival times and trading times, for it is the difference between an integral and its $\Delta$-discrete counterpart, a summation. The third term is the expected variance of the change in the efficient allocations per unit of time, $\mu \sigma_{z}^{2}$, multiplied by the expected waiting time until the dividend is paid, $1 / r$. Note that the misalignment term only involves the Poisson information arrivals after time 0 , since the first information arrival time coincides with the first trading time, time 0 . The misalignment term and hence $X_{2}(\Delta)$ vanish as $\Delta \rightarrow 0$, i.e., there is zero welfare cost from trading delay if trading is continuous. 
The total inefficiency of the strategic equilibrium is

$$
X(\Delta) \equiv X_{1}(\Delta)+X_{2}(\Delta)=\frac{\lambda}{2 r} \sigma_{z}^{2}\left[\frac{\mu}{r}+1-\left(1-\frac{1+d}{n \alpha-1}\right)\left(1+\frac{\Delta e^{-r \Delta}}{1-e^{-r \Delta}} \mu\right)\right]
$$

We see that under Poisson news arrivals both terms $X_{1}(\Delta)$ and $X_{2}(\Delta)$ are generally positive. We thus expect an interior optimal $\Delta^{*}$.

The following proposition, also the main result of this subsection, characterizes the optimal trading frequency in the case of ex-ante identical traders.

Proposition 7. Suppose that $\left\{T_{k}\right\}_{k \geq 1}$ is a Poisson process with intensity $\mu$, and $z_{i, 0}=Z / n$ for every trader $i$ (i.e., $\sigma_{0}^{2}=\sigma_{z}^{2}$ ). Then the following hold.

1. For any $l \geq 2 / 3$, we have $\Delta^{*}<1 /(l \mu)$ if $n \alpha>2 l+2 / 3$. In particular, we always have $\Delta^{*}<1.5 / \mu$ since $n \alpha>2$ by assumption.

2. $\Delta^{*}$ strictly decreases in $\mu, n$ and $\sigma_{w}^{2}$, and strictly increases in $\sigma_{D}^{2}$.

Part 1 of Proposition 7 establishes a sufficient condition under which the optimal trading frequency $1 / \Delta^{*}$ is more than $l$ times as high as the information frequency $\mu$. For reasonable $l$, the condition $n \alpha>2 l+2 / 3$ is not stringent. For "liquid" assets, namely those with wide investor participation (large $n$ ), low information asymmetry (low $\sigma_{D}^{2}$ ), and high liquidity-driven trading motives (high $\sigma_{w}^{2}$ ), we expect $\alpha$ to be much closer to 1 than to $2 / n$. As extreme examples, $\alpha=1$ if $\sigma_{D}^{2}=0$ (pure private value) or if $\sigma_{\epsilon}^{2}=0$ (public information of common value). These liquid assets include major equity indices, government securities, and foreign currencies, as well as the corresponding futures contracts. As a concrete example, Joint Staff Report (2015) finds that about 80 bank-dealers and principal trading firms actively participate in the cash U.S. Treasury markets. An $n \alpha$ of 80 means that the optimal trading frequency in U.S. Treasury market should be at least 40 times as high as the information arrival frequency.

To get an intuitive sense of how the lower bound in Proposition 7 is derived, we provide a heuristic proof when $n \alpha$ is close to 2 . As $n \alpha \rightarrow 2$, bid shading is the most severe, and traders benefit the most from a large $\Delta$ to increase the market depth in each period. We now show that even in this case the optimal $\Delta^{*}<1.5 / \mu$, corresponding to $l=2 / 3$.

From Equation (52), we see that minimizing $X(\Delta)$ over $\Delta$ is equivalent to maximizing

$$
\left(1-\frac{1+d}{n \alpha-1}\right)\left(1+\frac{\mu \Delta e^{-r \Delta}}{1-e^{-r \Delta}}\right)=\left(1-\frac{\sqrt{\left(1-e^{-r \Delta}\right)^{2}+4 e^{-r \Delta} y}-\left(1-e^{-r \Delta}\right)}{2 e^{-r \Delta}}\right)\left(1+\frac{\mu \Delta e^{-r \Delta}}{1-e^{-r \Delta}}\right)
$$


where $y \equiv \frac{1}{(n \alpha-1)^{2}}$ and we have used the expression of $d$ in Equation (32). As $y \rightarrow 1$ from below (since $n \alpha \rightarrow 2$ from above), by Taylor's theorem Equation (53) becomes:

$$
\frac{1}{1+e^{-r \Delta}}\left(1+\frac{\mu \Delta e^{-r \Delta}}{1-e^{-r \Delta}}\right) \cdot O(1-y)
$$

We have:

$$
\frac{\partial}{\partial \Delta}\left(\frac{1}{1+e^{-r \Delta}}\left(1+\frac{\mu \Delta e^{-r \Delta}}{1-e^{-r \Delta}}\right)\right)=\frac{\mu\left(e^{r \Delta}-e^{-r \Delta}\right)-r(\mu \Delta-1)\left(e^{r \Delta}+e^{-r \Delta}\right)-2 r}{\left(e^{r \Delta}-e^{-r \Delta}\right)^{2}} \equiv \frac{f(\Delta)}{\left(e^{r \Delta}-e^{-r \Delta}\right)^{2}} .
$$

Clearly, $f^{\prime}(\Delta)<0$ if $\Delta>1 / \mu$. Moreover, by applying the hyperbolic inequality

$$
\frac{\sinh (x)}{x}<\frac{2}{3}+\frac{1}{3} \cosh (x), \quad x>0
$$

it is straightforward to show that $f(1.5 / \mu)<0$. Thus, the $\Delta^{*}$ that maximizes Equation (54) is strictly less than $1.5 / \mu$ for any $\mu>0$ and $r>0$.

A comparison between Part 1 of Proposition 7 and Proposition 5 reveals the major difference between scheduled and stochastic information arrivals. Take the case of ex-ante identical traders. Under scheduled information arrivals, the optimal trading frequency is equal to the information arrival frequency. Under stochastic information arrival, the optimal trading frequency can be much higher than the information arrival frequency.

Part 2 of Proposition 7 can be proven by applying the monotone comparative statics of Milgrom and Shannon (1994) and Zandt (2002). For example, by direct calculation one can show that

$$
\frac{\partial^{2} X(\Delta)}{\partial \Delta \partial \mu}=-\frac{\partial}{\partial \Delta}\left[\left(1-\frac{1+d}{n \alpha-1}\right) \frac{\Delta e^{-r \Delta}}{1-e^{-r \Delta}}\right]>0 .
$$

Thus, $\Delta^{*}$ is strictly decreasing in $\mu$. Intuitively, if information arrives more frequently, the optimal trading frequency should also increase in order to allow traders faster response to new information.

By the same method, we can show that the optimal $\Delta^{*}$ is strictly decreasing in $n \alpha$. Since $\alpha$ is the weight on one's own total signal, $\alpha$ is strictly increasing in the variance of private-value shocks, $\sigma_{w}^{2}$, and is strictly decreasing in the variance of common-value shocks, $\sigma_{D}^{2}$. Hence, $\Delta^{*}$ is also strictly decreasing in $\sigma_{w}^{2}$ and is strictly increasing in $\sigma_{D}^{2}$. The intuition is that if trading is motivated less by informed speculation and more by private values, then traders will submit more aggressive demand schedules anyway; in those situations a higher-frequency market is better because it reduces delays in responding to new information. Moreover, we can show that $n \alpha$ is strictly increasing in $n$ (even though $\alpha$ itself decreases with $n$ ), so $\Delta^{*}$ is strictly decreasing in $n$. 
Figure 5: Comparative statics for $\Delta^{*}$ when all traders are ex-ante identical (i.e. $\sigma_{0}^{2}=\sigma_{z}^{2}$ ). The solid lines plot the optimal $\Delta^{*}$, and the dashed lines plot the mean waiting time for Poisson information $1 / \mu$. Baseline parameters: $n=10, r=1 / 22, \mu=1, \lambda=1 / 1000, \sigma_{D}^{2}=1, \sigma_{\epsilon}^{2}=1$, and $\sigma_{w}^{2}=1 / 10$.
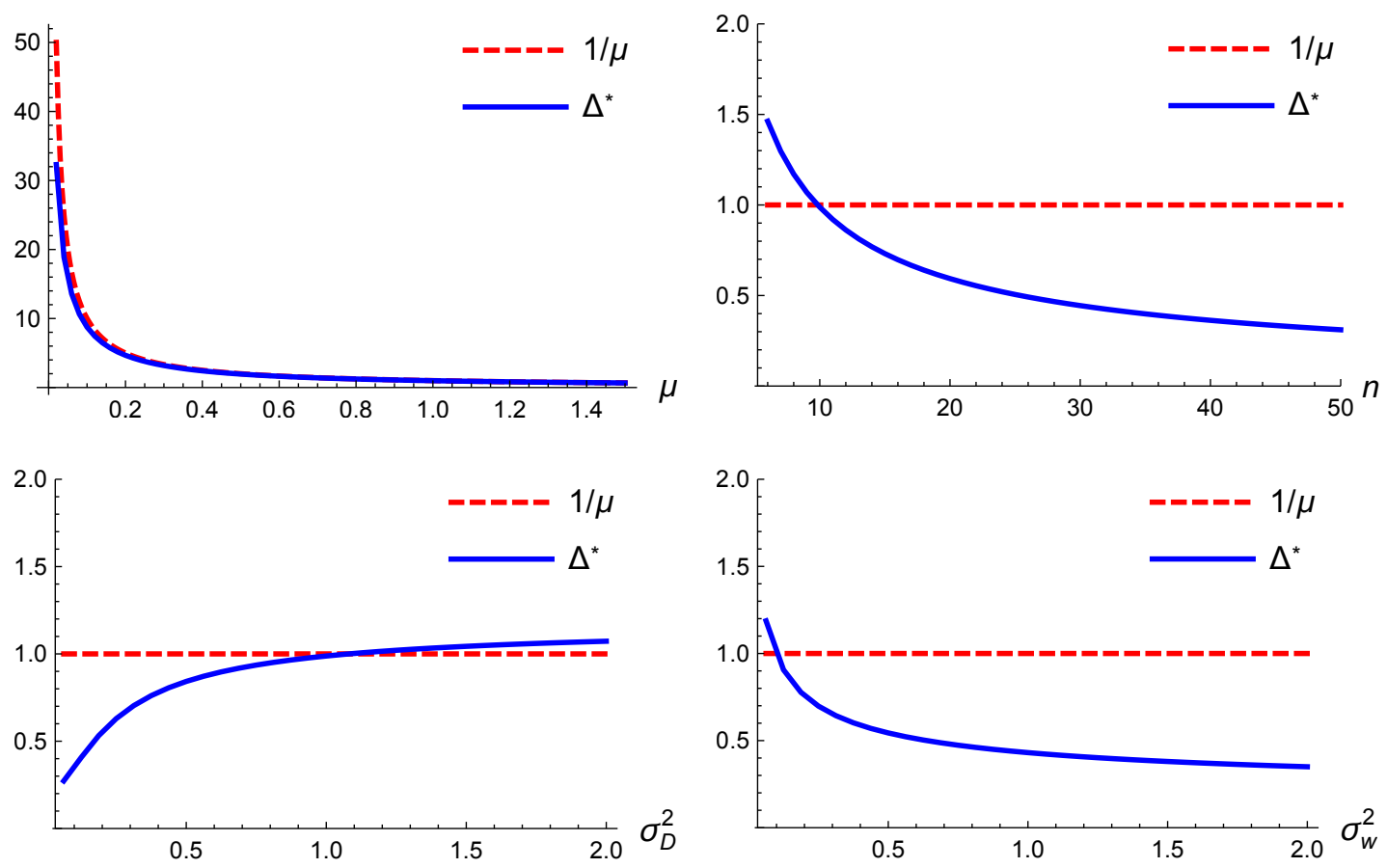

Figure 5 plots the optimal $\Delta^{*}$ for a variety of model parameters and illustrates the comparative statics. From the top-right subplot we also see that the bound $\Delta^{*}<1.5 / \mu$ is actually fairly tight: while $\mu$ is kept at one, $\Delta^{*}$ is just below 1.5 when $n=6$. In general, $\Delta^{*}$ can be substantially lower than $1 / \mu$.

Overall, Proposition 7 predicts that the optimal trading frequency of an asset should increases in its "liquidity," measured as a higher participation of investors, a higher private-value (e.g. hedging) motive for trades, and a lower adverse selection. For relatively liquid assets like stock indices, large stocks, government securities, foreign exchange, and futures contracts, the optimal trading frequency could be substantially higher than the arrival frequency of information that affects these assets. For relatively illiquid assets like small stocks, corporate bonds, municipal bonds, and nonstandard over-the-counter derivatives, the optimal trading frequency can be lower, but only moderately lower, than the arrival frequency of relevant information.

We close this section with the following proposition on comparative statics when $\sigma_{0}^{2}$ and $\sigma_{z}^{2}$ are potentially different.

Proposition 8. Suppose that $\left\{T_{k}\right\}_{k \geq 1}$ is a Poisson process with intensity $\mu$. The following comparative statics hold: 
1. If $\sigma_{0}^{2}>0$, then $\Delta^{*}$ strictly decreases in $\mu$ from $\infty($ as $\mu \rightarrow 0)$ to $0($ as $\mu \rightarrow \infty)$.

2. If $\sigma_{0}^{2} / \sigma_{z}^{2}$ remains bounded as $n \rightarrow \infty$, then $\Delta^{*} \rightarrow 0$ as $n \rightarrow \infty$.

The intuition for Proposition 8 is similar to that of Proposition 7. In particular, continuous trading becomes optimal in the limit as the market becomes large or as the arrival rate of new information increases without bound.

\section{Heterogeneous Trading Speeds}

In this section we extend the model to study trading strategies and welfare if traders have heterogeneous speeds. In this extension, speed is defined by how frequently a trader participates in the market. The main result of this section is that traders who can access the market all the time, such as high-frequency traders or financial intermediaries, prefer the highest trading frequency, but traders who access the market infrequently, such as retail and institutional investors, typically prefer a strictly lower trading frequency. These results reveal that a market speed-up has differential effects on heterogeneous groups of market participants. To highlight this new insight and for tractability, we shall simplify other aspects of the model to its minimum.

\subsection{Model setup}

As before, trading happens in the form of double auctions at times $\{0, \Delta, 2 \Delta, \ldots\}$. There are $n_{F} \geq 1$ ex-ante identical fast traders who trade in every period $t \geq 0$. There are $M$ ex-ante identical slow traders who can also trade in the market repeatedly, but each access to market comes with a deterministic delay of clock time $\Gamma>0$. We interpret $\Gamma$ as the fundamental delay of slow investors that is not affected by the market frequency $1 / \Delta$. For simplicity, at time 0 , the arrival sequence of the $M$ slow traders are drawn at random, and their first arrival times are $\Gamma / M, 2 \Gamma / M, 3 \Gamma / M, \ldots, \Gamma$. By the deterministic nature of delays between market accesses, ${ }^{10}$ the $j$-th slow trader comes to the market at clock times $\frac{j}{M} \Gamma, \frac{j}{M} \Gamma+\Gamma, \frac{j}{M} \Gamma+2 \Gamma, \ldots$, where $j \in\{1,2, \ldots, M\}$. For instance, many retail and institutional investors rebalance their portfolios infrequently, and their rebalancing delay $\Gamma$ is insensitive to the trading delay $\Delta$ in the market.

We assume that the slow traders cannot coordinate their trading times, ${ }^{11}$ so each slow

\footnotetext{
${ }^{10}$ We could also use a random delay, but this alternative arrangement would not change our results.

${ }^{11}$ In many markets, this assumption is not restrictive. For example, in the U.S. equity markets, when retail or institutional investors send orders to their brokers, the brokers' best-execution responsibility is to get the best price in the market. Delays could be beneficial, but the executing brokers would incur the risk that the stock prices move in the adverse direction while waiting. The resulting adverse price movement, or "implementation shortfall," would count against the brokers. Therefore, if a broker receives a marketable order that creates little
} 
Fig

auc

(bli

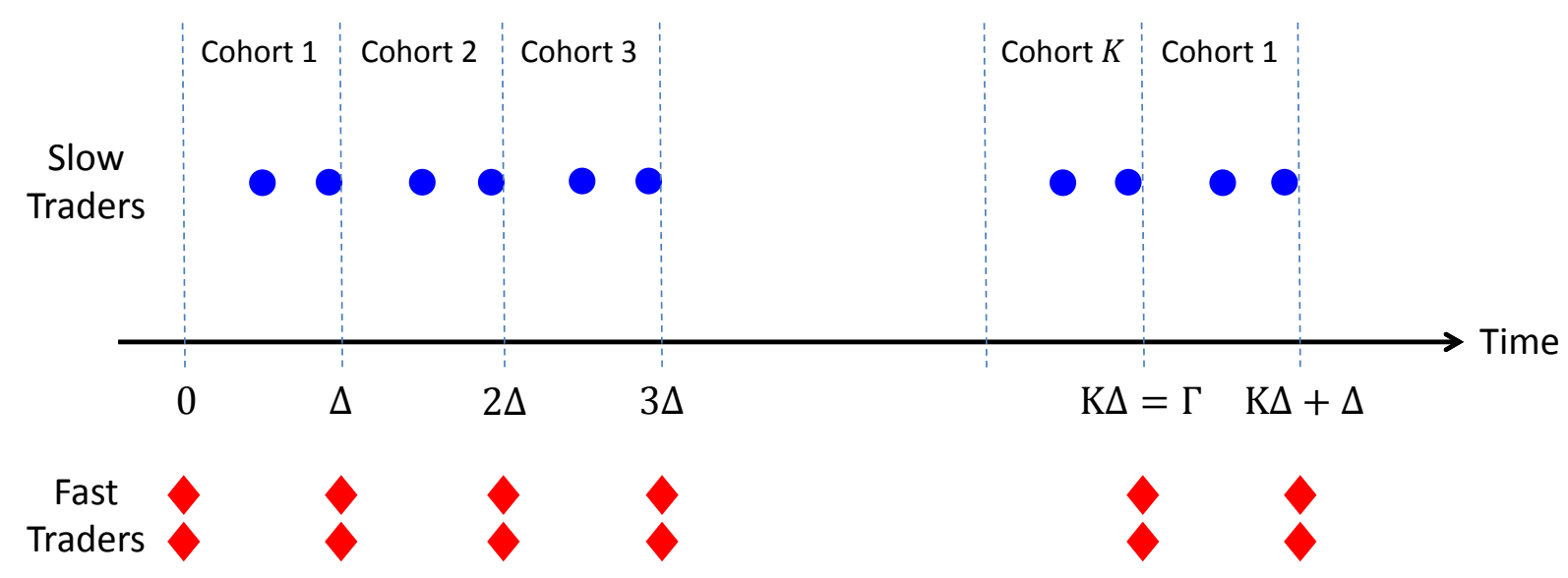

trader trades in the immediate next double auction after his arrival or return to the market. Let $K=\Gamma / \Delta$, that is, it takes $K$ double auctions to make sure that each slow trader has one chance to trade. For simplicity, we will consider values of $\Delta$ such that $K$ is a positive integer. The $M$ slow traders are naturally separated into $K$ "cohorts," and each cohort has $n_{S} \equiv M / K$ slow traders. The $k$-th cohort of $n_{S}$ slow traders trade with the $n_{F}$ fast traders at times $k \Delta$, $(K+k) \Delta,(2 K+k) \Delta$, etc. Because each slow traders participates only in $1 / K$ of all double auctions and fast traders participate in all auctions, we can say that a fast trader is $K$ times as fast as a slow trader. When $K=1$, we get the homogeneous speed model in Section 2 .

Figure 6 illustrates the time line of the heterogeneous-speed market with two fast traders (red diamonds) and two slow traders per period (blue circles).

In this market, a higher trading frequency, or a lower $\Delta$, creates the following tradeoff for the slow traders. On the one hand, a lower $\Delta$ reduces the average waiting time of each cohort of slow traders. Specifically, within each cohort, the first trader's waiting time before trading is $\frac{n_{S}-1}{n_{S}} \Delta$, and the last trader's waiting time is zero. The average waiting time per slow trader is thus $\frac{n_{S}-1}{2 n_{S}} \Delta$. If $\Delta$ is reduced by, say, a half, then the average waiting time is also reduced to $\frac{n_{S} / 2-1}{2 \times n_{S} / 2} \frac{\Delta}{2}=\frac{n_{S} / 2-1}{2 n_{S}} \Delta$. A shorter waiting time is therefore a benefit of a higher trading frequency. On the other hand, a higher trading frequency also reduces the number of slow traders $n_{S}$ in each double auction, since $n_{S}=M \Delta / \Gamma$. (This claim relies on the assumption that slow

price impact, sending it immediately to the market minimizes such risk. The qualitative nature of our results still applies as long as slow traders cannot perfectly coordinate their trading times. If the slow traders were able to coordinate their trading times perfectly, they effectively dictate the trading frequency of the market and implement their preferred frequency $1 / \Delta_{S}^{*}$ that we characterize shortly. 
traders cannot perfectly coordinate their trading times.) This implies that slow traders and fast traders in each auction tend to be less aggressive, resulting in slower convergence to the efficient allocation. We see that this fundamental tradeoff is very similar to that in Section 3.

As before, a divisible asset pays a liquidating dividend $D$ at an exponentially-distributed future time with mean $1 / r$. To isolate the main insight of this section about heterogeneous speeds, we use a much simpler information structure. Specifically, fast and slow traders receive no signals about the dividend $D$; thus, they all value the dividend at $\mathbb{E}[D]$, which is normalized to zero. Besides tractability, symmetric information regarding the asset fundamental value sets our model of this section orthogonal to recent models of high-frequency trading in which fast traders also have information advantage (see, for example, Biais, Foucault, and Moinas (2015), Jovanovic and Menkveld (2012), and Hoffmann (2014)).

We now describe the inventory evolution of the traders. We refer to a generic fast trader as $i$, and a generic slow trader as $j$. Each fast trader starts with an initial inventory of $z_{i, 0}=0$. A fast trader's inventory at time $\tau$ is:

$$
z_{i, \tau}=z_{i, t \Delta}+x_{i, t \Delta}^{*}, \quad \tau \in(t \Delta,(t+1) \Delta]
$$

where $x_{i, t \Delta}$ is the fast trader's demand schedule in period $t, p_{t \Delta}^{*}$ is the market-clearing price in period $t$, and $x_{i, t \Delta}^{*}=x_{i, t \Delta}\left(p_{t \Delta}^{*}\right)$. Since at time 0 no slow trader has arrived and all fast traders have the same inventory, $x_{i, 0}^{*}=0$ in the time- 0 double auction.

Without loss of generality, we consider a specific slow trader $j$ who belongs to cohort $k$. Let $T_{j, l} \in((l K+(k-1)) \Delta,(l K+k) \Delta]$ be the $l$-th arrival time when the slow trader $j$ comes to the market. When arriving for the first time, trader $j$ starts with an inventory $z_{j, T_{j, 0}}$ that is independently drawn from some mean-0 distribution. Subsequently, upon his $l$-th return, trader $j$ receives an inventory shock $\epsilon_{j, T_{j, l}}$, again independently drawn from another mean-0 distribution. The repeated inventory shocks make sure that inventory information is never common knowledge. Thus, slow trader $j$ 's inventory evolves according to:

$$
z_{j, \tau}=\left\{\begin{array}{ll}
0 & \tau \in\left[0, T_{j, 0}\right) \\
z_{j, T_{j, 0}} & \tau \in\left[T_{j, 0}, k \Delta\right] \\
z_{j,((l-1) K+k) \Delta}+x_{j,((l-1) K+k) \Delta}^{*} & \tau \in\left(((l-1) K+k) \Delta, T_{j, l}\right), l \geq 1 \\
z_{j,((l-1) K+k) \Delta}+x_{j,((l-1) K+k) \Delta}^{*}+\epsilon_{j, T_{j, l}}, & \tau \in\left[T_{j, l},(l K+k) \Delta\right], l \geq 1
\end{array} .\right.
$$

Note that slow trader $j$ only participates in double auctions held in period $(l-1) K+k$, for $l \geq 1$.

As before, before the asset payoff is realized, traders incur flow cost that is quadratic in 
inventories. Instead of having the same coefficient $\lambda$, fast traders and slow traders have potentially different coefficients $\lambda_{F}$ and $\lambda_{S}$, respectively. Inventory costs associated with uneven inventories are the source of gains from trade in this extension.

The expected utilities of fast trader $i$ and slow trader $j$ are, respectively:

$$
\begin{aligned}
& U_{i}=\mathbb{E}\left[-\int_{\tau=0}^{\infty} r e^{-r \tau} \frac{\lambda_{F}}{2 r} z_{i, \tau}^{2} d \tau-\sum_{t=0}^{\infty} e^{-r t \Delta} x_{i, t \Delta}^{*} p_{t \Delta}^{*}\right], \\
& U_{j}=\mathbb{E}\left[-\int_{\tau=0}^{\infty} r e^{-r \tau} \frac{\lambda_{S}}{2 r} z_{j, \tau}^{2} d \tau-\sum_{l=0}^{\infty} e^{-r(l K+k) \Delta} x_{j,(l K+k) \Delta}^{*} \cdot p_{(l K+k) \Delta}^{*}\right] .
\end{aligned}
$$

In these expressions we have used the normalization that the expected asset value $\mathbb{E}[D]=0$.

Each fast trader $i$ selects the optimal strategy profile $\left\{x_{i, t \Delta}\right\}$ to maximize $U_{i}$, and each slow trader $j$ selects the optimal strategy profile $\left\{x_{j,(l K+k) \Delta}\right\}$ to maximize $U_{j}$. The solution concept is still Perfect Bayesian Equilibrium from Definition 1.

\subsection{Equilibrium construction}

Let $\mathcal{C}(t \Delta)$ be the cohort of slow traders who trade at period $t$. Let

$$
Z_{F, t \Delta} \equiv \sum_{i=1}^{n_{F}}\left(z_{i, t \Delta}+x_{i, t \Delta}\left(p_{t \Delta}^{*}\right)\right)
$$

and

$$
Z_{S, t \Delta} \equiv \sum_{j \in \mathcal{C}(t \Delta)}\left(z_{j, t \Delta}+x_{j, t \Delta}\left(p_{t \Delta}^{*}\right)\right)
$$

be the total inventories of the fast traders and cohort- $t \Delta$ slow traders, respectively, after trading in period $t$. In period $t$, only one cohort of slow traders are present, and the total inventories of the other $K-1$ cohorts are $Z_{S,(t-1) \Delta}, Z_{S,(t-2) \Delta}, \ldots, Z_{S,(t-K+1) \Delta}$. For simplicity, we suppose that traders hold zero inventory before time 0 , that is, $Z_{F, 0}=0$ and $Z_{S,(1-k) \Delta}=0$ for $k \in$ $\{1,2, \ldots, K-1\}$. (We could also set the before-time-0 inventories to any vector of constants, but the analysis would not change in any substantial way.) 
We look for linear strategies of the form:

$$
\begin{aligned}
& x_{i, t \Delta}(p)=-b_{F} p+d_{F} z_{i, t \Delta}+e_{F} Z_{F,(t-1) \Delta}+\sum_{k=1}^{K-1} f_{F, k} Z_{S,(t-k) \Delta}, \\
& x_{j, t \Delta}(p)=-b_{S} p+d_{S} z_{j, t \Delta}+e_{S} Z_{F,(t-1) \Delta}+\sum_{k=1}^{K-1} f_{S, k} Z_{S,(t-k) \Delta} .
\end{aligned}
$$

That is, at period $t$, each trader's demand is linear in price, his own inventory, the fast traders' total inventory in period $t-1$, and the total inventory of each cohort of the slow traders except the current cohort. The equilibrium price in period $t$ is

$p_{t \Delta}^{*}=\frac{1}{n_{F} b_{F}+n_{S} b_{S}}\left(\left(d_{F}+n_{F} e_{F}+n_{S} e_{S}\right) Z_{F,(t-1) \Delta}+d_{S} \sum_{j \in \mathcal{C}(t \Delta)} z_{j, t \Delta}+\sum_{k=1}^{K-1}\left(n_{F} f_{F, k}+n_{S} f_{S, k}\right) Z_{S,(t-k) \Delta}\right)$.

A desirable property of strategies (64) and (65) is that the equilibrium path of prices $\left\{p_{t \Delta}^{*}\right\}$ reveal $\left\{\left(Z_{F, t \Delta}, Z_{S, t \Delta}\right)\right\}_{t \geq 0} \cdot{ }^{12}$

This equilibrium conjecture has one difficulty with off-equilibrium behavior. If a slow trader made a single deviation from strategy (65) in period $t, p_{t \Delta}^{*}$ would not reveal the true inventory of the cohort of slow traders present in period $t$. But the period- $t$ cohort does not participate in the market in the next $K-1$ periods, so prices $p_{(t+1) \Delta}^{*}, p_{(t+2) \Delta}^{*}, \ldots, p_{(t+K-1) \Delta}^{*}$ cannot provide any more information about the true inventory of the period- $t$ cohort. Therefore, cohorts of slow traders in periods $t+1, t+2, \ldots, t+K-1$ cannot learn the true inventory of the period- $t$ cohort through prices; they must form beliefs. From that point on, there could be an explosion in the number of state variables, including different traders' expectations of total inventories, their expectations of expectations, and so on. ${ }^{13}$

To avoid this intractable problem, we assume that all true total inventories are publicly and truthfully disclosed with a lag of $\Delta$. That is, at time $t \Delta, Z_{F,(t-1) \Delta}$ and $Z_{S,(t-k) \Delta}$ become common knowledge. Such public disclosure means that beliefs about the total inventory of fast traders or any cohort of slow traders are pin down by the true inventories regardless of the deviation by any single trader. Although this is a technical assumption, it can be motivated from post-

\footnotetext{
${ }^{12}$ To see this, consider the following inductive argument. At $t=1, Z_{F, 0}=0$ and $Z_{S,(1-k) \Delta}=0$ for $k=$ $1,2, \ldots, K-1$. The period-1 double auction price would then reveal $\sum_{j \in \mathcal{C}(\Delta)} z_{j, \Delta}$, from which we can calculate $Z_{F, \Delta}$ and $Z_{S, \Delta}$. In period $t>1$, given that $Z_{F,(t-1) \Delta}$ and $\left\{Z_{S,(t-k)}\right\}_{k=1}^{K-1}$ are known, $p_{t \Delta}^{*}$ reveals the current cohort of slow traders' total inventory $\sum_{j \in \mathcal{C}(t \Delta)} z_{j, t \Delta}$. Then given $p_{t \Delta}^{*}$, the fast and slow traders' new total inventory $Z_{F, t \Delta}$ and $Z_{S, t \Delta}$ can be calculated.

${ }^{13}$ In the homogeneous-speed model of Section 2 and Section 3, this problem does not arise because, after a trader's single deviation from the equilibrium strategy, the same trader still participates in subsequent double auctions. All other traders who did not deviate will infer the correct sum of total signals from prices and need not to form beliefs about it.
} 
trade transparency regulation observed in reality. For example, during various time periods, stock exchanges including Euronext Paris, the Tokyo Stock Exchange, the Korean Exchange, the Australian Securities Exchange, and the Helsinki Stock Exchange require that broker IDs be displayed together with their limit orders (see Comerton-Forde, Frino, and Mollica (2005), Foucault, Moinas, and Theissen (2007), Comerton-Forde and Tang (2009), and Linnainmaa and Saar (2012)).

Once the off-equilibrium-belief problem is circumvented this way, the equilibrium can be constructed by considering a single deviation of a trader and taking the first-order condition, just like in Section 2. Details of equilibrium construction are delegated to Appendix D.

\subsection{Preferred trading frequencies by fast traders and slow traders}

The equilibrium is analytically intractable, but it can be solved numerically. Using the firstorder conditions shown in Appendix D and matching coefficients, we can numerically solve the coefficients in Equations (64) and (65). From these strategies, we can subsequently evaluate the welfare of fast traders and slow traders.

Since the traders are heterogeneous, we ask what respective trading frequencies fast traders and slow traders prefer ex-ante. In other words, if fast traders dictate market design, what $\Delta$ would they choose ex-ante? The fast traders' optimal frequency is therefore defined by:

$$
\Delta_{F}^{*} \in \arg \max _{\Delta} \sum_{i=1}^{n_{F}} U_{i}
$$

Similarly, if slow traders dictate market design, they would choose their own optimal frequency, defined by:

$$
\Delta_{S}^{*} \in \arg \max _{\Delta} \sum_{j=1}^{M} U_{j} .
$$

Note that summing over individual traders' ex-ante welfare in the respective two groups is a valid welfare criterion, since traders in each group are ex-ante identical.

We numerically evaluate the ex-ante expected welfare of fast traders and slow traders for various levels of $K$, with the associated $\Delta=\Gamma / K$. A higher $K$ is is equivalent to a higher trading frequency $1 / \Delta$, which is equivalent to a lower number of slow traders per double auction. At the trading frequency $\Delta=\Gamma / M$, there is only one slow trader per double auction, and the market is the thinnest. If $\Delta$ were to get any smaller, say, $\Gamma /(2 M)$, there would be periods in which only fast traders participate in the double auctions. But because fast traders are identical in our model, on equilibrium path they have identical inventories period by period and will not trade any positive quantity with each other. In other words, setting a lower bound of $\Delta$ at 
$\Gamma / M$ is without loss of generality.

Figure 7 plots the fast traders' welfare, in red, and slow traders' welfare, in blue, as functions of $K \in\{1,2,4,8,16,32\}$. We take the following baseline parameters: $\lambda_{F}=\lambda_{S}=1, r=1$, $\Gamma=1, M=32$ and $n_{F}=2$. The corresponding $\Delta$ is in the finite set $\left\{\Gamma, \frac{\Gamma}{2}, \frac{\Gamma}{4}, \frac{\Gamma}{8}, \frac{\Gamma}{16}, \frac{\Gamma}{32}\right\}$. The inventory shocks $\left\{\epsilon_{j, T_{j, l}}\right\}$ are set to zeros to speed up numerical calculations. The initial inventories of the slow traders upon the first arrivals are normally distributed with mean zero and variance one. We also consider different levels of imminence of payoff-relevant news ( $r=1.5$ and $r=0.5)$ and different populations of slow traders $(M=64$ and $M=128)$. The optimal $K$ is indicated by a black dot.

The most salient result from Figure 7 is that the fast traders always prefer the highest $K$, or the highest possible trading frequency, at which only one slow trader participates in each double auction. Because it is already a corner solution, this result is not driven by our restricted attention to a discrete grid of $K$. Instead, the fast trader' preference for the highest trading frequency comes from the rents they extract by intermediating trades among slow traders, or market-making. Intuitively, the fewer slow traders per double auction, the higher rents extracted by fast traders because of imperfect competition. A higher trading frequency also reduces fast traders' inventory costs whenever their inventories are nonzero, since they can more quickly offload their inventories to the next cohort of slow traders. We emphasize that this result is not about superior information advantage of fast traders.

If there is only one fast trader $\left(n_{F}=1\right)$, we also find through numerical calculations that the fast trader's optimal trading frequency is such that there are two slow traders per period, which is also the highest feasible trading frequency. ${ }^{14}$ The results for $n_{F}=1$ are not reported to preserve space.

The result that fast traders prefer the highest possible trading frequency, or the thinnest market, is more robust than suggested by the numerical calculations. In the supplemental materials to this paper, we analytically solve a limiting case of our model, in which: (i) there is only one fast trader, and (ii) $M \rightarrow \infty, \Gamma \rightarrow \infty$, and $M / \Gamma$ converges to a positive constant. (In this limiting case, each slow trader trades only once.) We prove, analytically, that in this limiting case the fast trader always strictly prefers the highest feasible trading frequency of two slow traders per period.

Now turning to the slow traders, we observe that they typically prefer an intermediate trading frequency. ${ }^{15}$ Too low a trading frequency incurs too much waiting cost, whereas too

\footnotetext{
${ }^{14} \mathrm{~A}$ linear equilibrium does not exist if there are only two traders (one fast and one slow) in each period. This is consistent with the existing literature such as Kyle (1989), Vives (2011), and Rostek and Weretka (2012).

${ }^{15}$ Because the optimal $K$ for slow traders is intermediate, their optimal trading frequency is subject to the technical caveat that the welfare is calculated only for $K$ 's such that the number of slow traders per double auction is an integer. It is possible to calculate everything for a general $K$, but there is little gain in the economic
} 
Figure 7: Fast and slow traders' welfare as functions of $K$; the optimal $K$ is marked by a black point. Baseline parameters: $\lambda_{F}=\lambda_{S}=1, r=1, \Gamma=1, M=32$ and $n_{F}=2$. The inventory shocks $\epsilon_{j, T_{j, l}}$ are all set to zero. The initial inventories of the slow traders upon the first arrivals are normally distributed with mean zero and variance one.
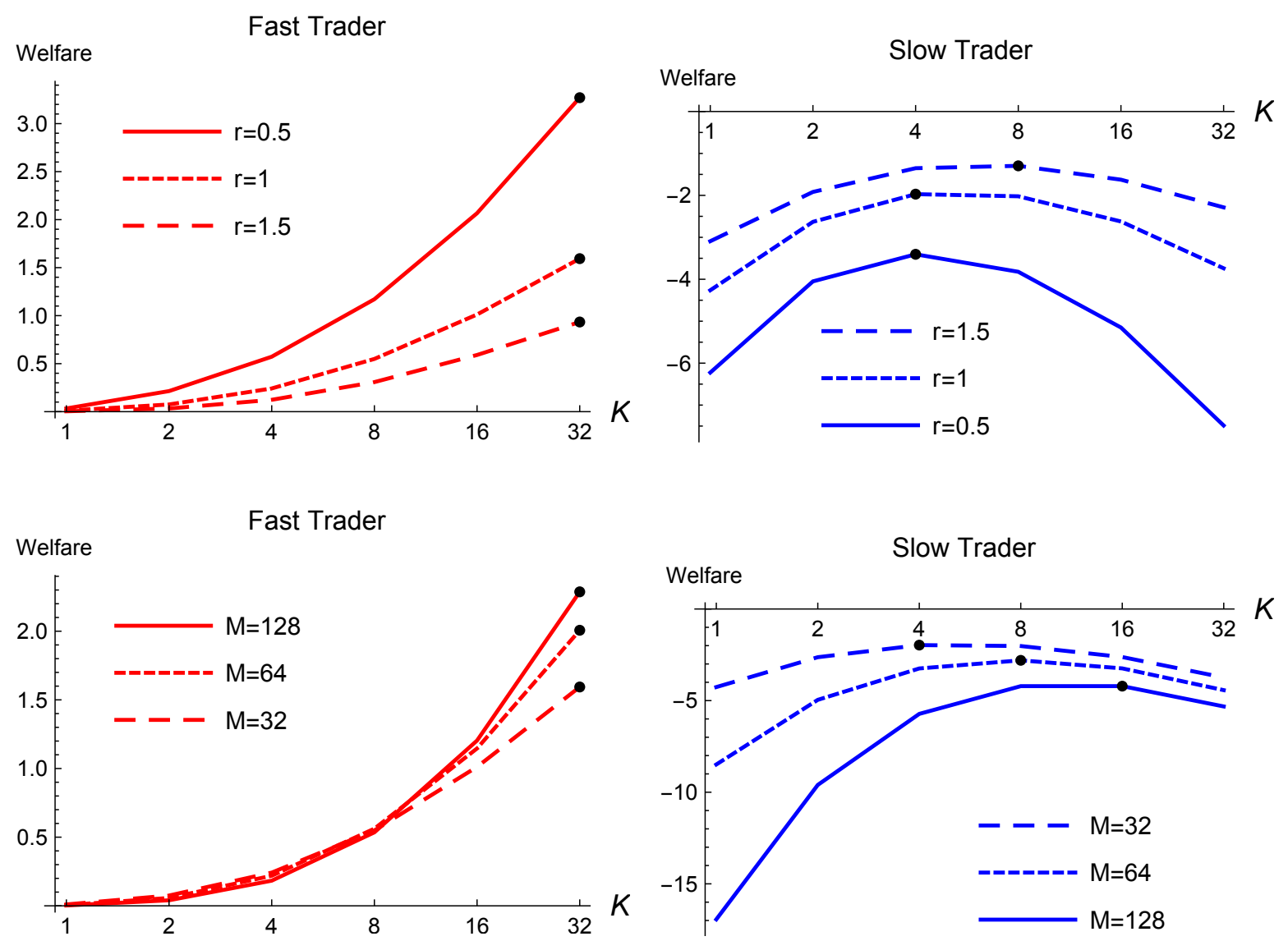

high a trading frequency makes each double auction too uncompetitive. The internal solution for $\Delta_{S}^{*}$ is obtained as a result of the tradeoff between shorter delays in offloading inventory shocks and a thicker, more competitive market. As the arrival rate $r$ of asset payoffs becomes smaller, slow traders are less eager to offload their inventories; thus, they prefer a thicker and slower market, i.e., a smaller $K$ and larger $\Delta$. As the population of slow traders becomes larger, each double auction becomes thicker automatically; consequently, slow traders prefer a faster market, i.e. a higher $K$ or smaller $\Delta$, to minimize delay costs. These comparative statics are natural. The case with $n_{F}=1$ yields qualitatively similar results for the slow traders.

An important implication from the heterogeneous-speed model is that when market participants are heterogeneous, who designs the market matters a great deal for the welfare of intuition. 
everyone. Mapping our fast and slow traders to their likely counterparts in reality, we see that financial intermediaries and high-frequency traders, likely the fast, benefit most from speeding up the market as much as possible, where retail and institutional investors, likely the slow, initially benefit from market speedup but eventually suffer from it. For more liquid securities, corresponding to a higher $M$, the fast traders' interest and the slow traders' interest are better aligned in speeding up the market. These implications are unique to the heterogeneous-speed model.

\subsection{Trading frequency, price discovery, and excess volatility}

We close this section with a simple observation implied by the model: under heterogeneous speeds, a higher-frequency market tends to create faster price discovery but higher short-term volatility. Intuitively, a higher trading frequency allows more immediate market reaction to supply or demand shocks, but due to market thinness and market power, the short-term price reaction "overshoots" the long-term price reaction.

Figure 8 illustrates this idea in an simple example, in which there are $n_{F}=2$ fast traders and $M=32$ slow traders, and all slow traders but one arrive with zero inventory. The 10th slow trader arrives with a large positive inventory of 20 units. Other parameters are the same as the baseline parameters used in Figure 7 . For each $K \in\{1,4,32\}$, the prices are calculated in the linear equilibrium characterized above, and the price paths are plotted as step functions in order to make clear the times of price changes. This exercise is obviously subject to the caveat that slow traders cannot coordinate their trading times. But again, as long as slow traders cannot perfectly coordinate trading times, the intuition would not change qualitatively.

At the slowest-market extreme of $K=1$, all 32 slow traders and two fast traders participate in the double auctions together. The 10th trader's supply shock is shared with the other 33 traders, so the price decline is mild but comes with a delay, at clock time 1.

At the fastest-market extreme of $K=32$, each double auction has only one slow trader and two fast traders. The 10th slow trader's large inventory creates a large price drop in the immediate next double auction. Price discovery is fast in that the price reflects the excess supply almost immediately. But the extent of price drop is much larger because (i) there are only two fast traders to share the inventory shock, and (ii) these two fast traders exercise market power. As soon as the 10th slow trader finishes the first round of trading, price rebounds sharply, and then gradually, as the two fast traders offload their newly acquired inventory to other slow traders in later periods. Because the 10th slow trader retains part of his original inventory, the price periodically drops and recovers as the 10th slow trader returns to the market 
Figure 8: Price responses to supply or demand shocks under various trading frequency. In this example, $M=32$, the 10th slow trader arrives with a positive inventory of 20 units, and other slow traders arrive with zero inventory. Other parameters are the same as the baseline parameters in Figure 7.

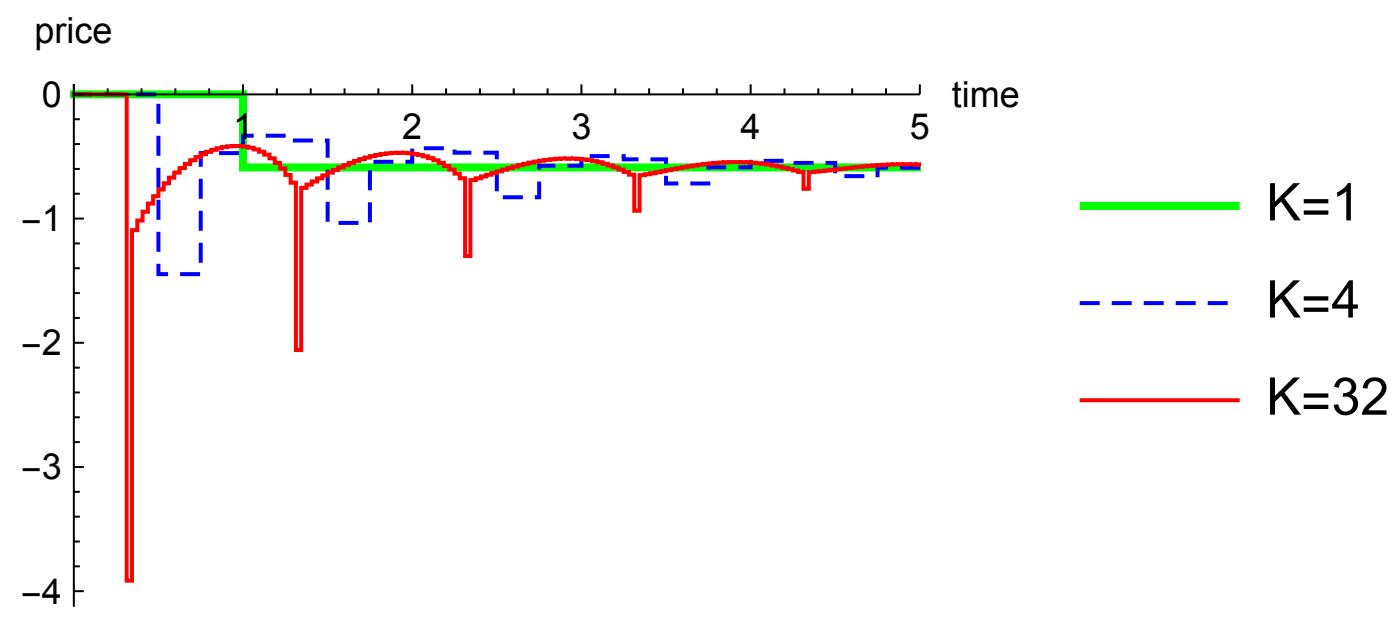

repeatedly. ${ }^{16}$ As time goes by, the price swing becomes smaller and smaller, and eventually the price converges to that under $K=1$. For $K=4$, the price exhibits the same drop-and-recover patterns but is less volatile.

These price patterns of Figure 8 nicely mirror the price patterns obtained in the model of Duffie (2010) based on investor inattention. Both models produce sharp price responses to supply or demand shocks and subsequent reversals, as well as later repercussions with smaller magnitudes. Beyond Duffie's model, our model features imperfect competition, so price reactions are more pronounced.

Overall, this simple application of our heterogeneous-speed model suggests that under imperfect competition, a higher market speed may lead to more frequent occurrence of (mini) "flash crashes" and "flash rallies" in electronic markets - sharp price drop and rebound that last for a short period of time, or vice versa. CFTC and SEC (2010), Kirilenko, Kyle, Samadi, and Tuzun (2015), Menkveld and Yueshen (2015), and Joint Staff Report (2015) provide indepth analysis of such events in markets for equities, futures, U.S. Treasuries, and foreign exchange.

\footnotetext{
${ }^{16}$ The deterministic time intervals between price drops come from the assumed deterministic trading delays. The model of Duffie (2010) has the same feature. Stochastic time intervals between price drops may be obtained by considering an alternative model in which each slow trader revisits the market according to a stochastic process, say a Poisson process. That alternative model does not offer new economic intuition beyond our current model, and is technically messier because the number of trader per double auction would be a random variable.
} 


\section{Conclusion}

In this paper, we study a dynamic model in which a finite number of traders receive private information over time and trade strategically with demand schedules in a sequence of double auctions. We characterize a stationary linear equilibrium in closed form. The equilibrium price aggregates a weighted sum of the common value information and private values information, but the two components cannot be separated from the price. Due to imperfect competition, the equilibrium allocation is not fully efficient, but it converges to the efficient allocation exponentially over time. The presence of adverse selection - asymmetric information regarding the common-value component of the asset - slows down this convergence speed.

We use this modeling framework to study the optimal trading frequency that maximizes welfare. Trading frequency is measured as the number of double auctions per unit of clock time. A higher trading frequency reduces the aggressiveness of demand schedules, but allows more immediate reactions to new information. The allocative inefficiency in this dynamic market can be decomposed into two parts: one part due to strategic behavior and the other due to delayed responses to new information. The optimal tradeoff between these two effects depends on the nature of information. If new information arrives at scheduled intervals, the optimal trading frequency is never higher than the information frequency, and these two frequencies coincide if traders are ex-ante identical. By contrast, if new information arrives as a Poisson process, the optimal trading frequency can be (much) higher than the information arrival frequency, unless adverse selection is very severe or if the number of traders is very low.

We extend the model to study how market frequency interacts with heterogeneous trading speeds. Fast traders access the market whenever it is open, but slow traders can only trade periodically with a delay. Fast trader extract rents by intermediating trades among slow traders across time. As a result, fast traders prefer the highest feasible trading frequency, whereas slow traders tend to prefer a strictly lower frequency. Under heterogeneous speeds and market power, a higher trading frequency speeds up price discovery, but may create higher short-term volatility and price patterns that resemble "flash crashes" and "flash rallies." 


\section{A List of Model Variables}

\begin{tabular}{|c|c|}
\hline Variable & Explanation \\
\hline & Sections 2-3, Exogenous Variables \\
\hline$t$ & Discrete trading period,$t \in\{0,1,2,3, \ldots\}$ \\
\hline$\tau$ & Continuous clock time, $\tau \in[0, \infty)$ \\
\hline$\Delta$ & Length of each trading period \\
\hline $\mathcal{T}, r$ & $\begin{array}{l}\text { The clock time } \mathcal{T} \text { of dividend payment has an exponential distribution with } \\
\text { intensity } r>0 \text {. }\end{array}$ \\
\hline$\left\{T_{k}\right\}_{k \in\{0,1,2, \ldots\}}$ & Times of shocks to the common dividend and private values \\
\hline$D_{T_{k}}$ & The common dividend value immediately after the $k$-th shock \\
\hline$\sigma_{D}^{2}$ & Each dividend shock $D_{T_{k}}-D_{T_{k-1}}$ has the distribution $\mathcal{N}\left(0, \sigma_{D}^{2}\right)$. \\
\hline$S_{i, T_{k}}$ & Trader $i$ 's signal of the $k$-th dividend shock \\
\hline$\sigma_{\epsilon}^{2}$ & $\begin{array}{l}\text { The noise in trader } i \text { 's dividend signal regarding the } k \text {-th dividend shock, } \\
S_{i, T_{k}}-\left(D_{T_{k}}-D_{T_{k-1}}\right) \text {, has the distribution } \mathcal{N}\left(0, \sigma_{\epsilon}^{2}\right) \text {. }\end{array}$ \\
\hline$w_{i, T_{k}}$ & Trader $i$ 's private value for the asset immediately after the $k$-th shock \\
\hline$\sigma_{w}^{2}$ & $\begin{array}{l}\text { Shocks to each trader } i \text { 's private value, } w_{i, T_{k}}-w_{i, T_{k-1}} \text {, has the distribution } \\
\mathcal{N}\left(0, \sigma_{w}^{2}\right) \text {. }\end{array}$ \\
\hline$v_{i, \tau}$ & $D_{T_{k}}+w_{i, T_{k}}$ if $T_{k}$ is the last shock before $\tau$ \\
\hline$\lambda$ & $\begin{array}{l}\text { Before the dividend is paid, the flow cost for holding asset position } q \text { is } \\
0.5 \lambda q^{2} \text { per unit of clock time for each trader. }\end{array}$ \\
\hline$Z$ & The total inventory held by all traders, $Z \equiv \sum_{1 \leq j \leq n} z_{j, 0}$ \\
\hline$\gamma$ & Time interval of scheduled information arrivals \\
\hline \multirow[t]{2}{*}{$\mu$} & Intensity of stochastic information arrivals \\
\hline & Sections 2-3, Endogenous Variables \\
\hline$z_{i, t \Delta}$ & Trader $\bar{i}$ 's inventory level right before the period $-t$ double auction \\
\hline$x_{i, t \Delta}(p)$ & Trader $i$ 's demand schedule in the period- $t$ double auction \\
\hline$p_{t \Delta}^{*}$ & The equilibrium price in period- $t$ double auction \\
\hline$H_{i, t \Delta}$ & $\begin{array}{l}\text { Trader } i \text { 's history (information set) up to time } t \Delta \text { but before the period- } t \\
\text { double auction, defined in Equation (10) }\end{array}$ \\
\hline$s_{i, T_{k}}$ & Trader $i$ 's total signal right after the $k$-th shock, defined in Equation (20) \\
\hline$V_{i, t \Delta}$ & The expected utility of trader $i$ in period $t$, conditional on $H_{i, t \Delta}$ \\
\hline$\chi, \alpha$ & Constants defined in Section 2.2 \\
\hline$z_{i, t \Delta}^{c}$ & The competitive allocation immediately before trading in period- $t$ auction \\
\hline$z_{i, \tau}^{e}$ & The continuous-time version of competitive allocation \\
\hline$\sigma_{z}^{2}, \sigma_{0}^{2}$ & Constants defined in Equations (46) and (47) \\
\hline$W(\Delta)$ & Welfare under homogeneous speed and trading interval $\Delta$ \\
\hline \multicolumn{2}{|r|}{$\underline{\text { Section } 4}$} \\
\hline$n_{F}, M$ & The number of fast traders and slow traders, respectively \\
\hline$\Gamma$ & Clock time delay of slow traders in repeated participation in the market \\
\hline$K$ & Number of cohort of slow traders, $K=\Gamma / \Delta$ \\
\hline$n_{S}$ & The number of slow traders in each cohort, $n_{S}=M / K$ \\
\hline$W_{F}(\Delta), W_{S}(\Delta)$ & The fast and slow traders' welfare, respectively \\
\hline$\Delta_{F}^{*}, \Delta_{S}^{*}$ & The fast and slow traders' preferred trading frequencies, respectively \\
\hline
\end{tabular}




\section{B Additional Results}

\section{B.1 The Continuous-Time Limit of Proposition 2}

In this appendix we examine the limit of the equilibrium in Proposition 2 as $\Delta \rightarrow 0$ (i.e. as trading becomes continuous in clock time) and its efficiency properties.

Proposition 9. As $\Delta \rightarrow 0$, the equilibrium of Proposition 2 converges to the following perfect Bayesian equilibrium:

1. Trader $i$ 's equilibrium strategy is represented by a process $\left\{x_{i, \tau}^{\infty}\right\}_{\tau \geq 0}$. At the clock time $\tau, x_{i, \tau}^{\infty}$ specifies trader $i$ 's rate of order submission and is defined by

$$
x_{i, \tau}^{\infty}\left(p ; s_{i, \tau}, z_{i, \tau}\right)=b^{\infty}\left(s_{i, \tau}-p-\frac{\lambda(n-1)}{r(n \alpha-1)} z_{i, \tau}+\frac{\lambda(1-\alpha)}{r(n \alpha-1)} Z\right)
$$

where

$$
b^{\infty}=\frac{r^{2}(n \alpha-1)(n \alpha-2)}{2 \lambda(n-1)} .
$$

Given a clock time $\tau>0$, in equilibrium the total amount of trading by trader $i$ in the clock-time interval $[0, \tau]$ is

$$
z_{i, \tau}^{*}-z_{i, 0}=\int_{\tau^{\prime}=0}^{\tau} x_{i, \tau^{\prime}}^{\infty}\left(p_{\tau^{\prime}}^{*} ; s_{i, \tau^{\prime}}, z_{i, \tau^{\prime}}^{*}\right) d \tau^{\prime}
$$

2. The equilibrium price at any clock time $\tau$ is

$$
p_{\tau}^{*}=\frac{1}{n} \sum_{i=1}^{n} s_{i, \tau}-\frac{\lambda}{n r} Z .
$$

3. Given any $0 \leq \underline{\tau}<\bar{\tau}$, if $s_{i, \tau}=s_{i, \underline{\tau}}$ for all $i$ and all $\tau \in[\underline{\tau}, \bar{\tau}]$, then the equilibrium inventories $z_{i, \tau}^{*}$ in this interval satisfy:

$$
z_{i, \tau}^{*}-z_{i, \underline{\tau}}^{e}=e^{-\frac{1}{2} r(n \alpha-2)(\tau-\underline{\tau})}\left(z_{i, \underline{\tau}}^{*}-z_{i, \underline{\tau}}^{e}\right),
$$

where

$$
z_{i, \underline{\tau}}^{e} \equiv \frac{r(n \alpha-1)}{\lambda(n-1)}\left(s_{i, \underline{\tau}}-\frac{1}{n} \sum_{j=1}^{n} s_{j, \underline{\tau}}\right)+\frac{1}{n} Z
$$

is the efficient allocation at clock time $\underline{\tau}$ (cf. Equation (26)).

Proof. The proof follows by directly calculating the limit of Proposition 2 as $\Delta \rightarrow 0$ using L'Hopitâl's rule.

Proposition 9 reveals that even if trading occurs continuously, in equilibrium the competitive allocation is not reached instantaneously. The delay comes from traders' price impact and the associated demand reduction. This feature is also obtained by Vayanos (1999). Although submitting aggressive orders allows a trader to achieve his desired allocation sooner, aggressive bidding also moves the price against the trader and increases his trading cost. Facing this tradeoff, each trader uses a finite rate of order submission in the limit. As in Proposition 4, the rate of convergence to the competitive allocation in Proposition 9, $r(n \alpha-2) / 2$, is increasing in $n, r$, and $\sigma_{w}^{2}$ but decreasing in $\sigma_{D}^{2}$. (The proof of Proposition 4 shows that $\partial(n \alpha) / \partial \sigma_{w}^{2}>0, \partial(n \alpha) / \partial \sigma_{D}^{2}<0$, and $\partial(n \alpha) / \partial n>0$.) 


\section{B.2 Convergence rate to efficiency in large markets}

To further explore the effect of adverse selection for allocative efficiency, and to compare with the literature (in particular with Vayanos (1999)), we consider the rate at which inefficiency vanishes as the number of traders becomes large, with and without adverse selection. Adverse selection exists if $\sigma_{D}^{2}>0$ and $\sigma_{\epsilon}^{2}>0$. For fixed $\sigma_{\epsilon}^{2}>0$ and $\sigma_{w}^{2}>0$, we compare the convergence rate in the case of a fixed $\sigma_{D}^{2}>0$ to that in the case of $\sigma_{D}^{2}=0$.

We consider the inefficiency caused by strategic behavior, that is, the difference between the total ex-ante utility in the strategic equilibrium of Proposition 2 and the total ex-ante utility in the competitive equilibrium:

$$
\begin{aligned}
X_{1}(\Delta) \equiv \mathbb{E}\left[\sum_{t=0}^{\infty}\left(e^{-r t \Delta}-e^{-r(t+1) \Delta}\right) \sum_{i=1}^{n}(\right. & \left(v_{i, t \Delta} z_{i,(t+1) \Delta}^{*}-\frac{\lambda}{2 r}\left(z_{i,(t+1) \Delta}^{*}\right)^{2}\right) \\
& \left.\left.-\left(v_{i, t \Delta} z_{i,(t+1) \Delta}^{c}-\frac{\lambda}{2 r}\left(z_{i,(t+1) \Delta}^{c}\right)^{2}\right)\right)\right],
\end{aligned}
$$

where $\left\{z_{i,(t+1) \Delta}^{*}\right\}$ is strategic allocation given by Equation (34), and $z_{i,(t+1) \Delta}^{c}$ is the competitive allocation given by Equation (26). This $X_{1}(\Delta)$ is the same as that defined in Section 3. As usual, prices do not enter the welfare criterion as they are transfers.

Proposition 10. Suppose that the news times $\left\{T_{k}\right\}_{k \geq 1}$ either satisfy $T_{k}=k \gamma$ for a constant $\gamma>0$ or are given by a homogeneous Poisson process. Suppose also that $\sigma_{\epsilon}^{2}>0, \sigma_{w}^{2}>0$, and $\frac{1}{n} \sum_{i=1}^{n} \mathbb{E}\left[\left(z_{i, 0}-\right.\right.$ $\left.\left.z_{i, 0}^{e}\right)^{2}\right]$ is bounded as $n$ becomes large. Then, the following convergence results hold:

1. If $\sigma_{D}^{2}>0$, then as $n \rightarrow \infty$ :

$$
\begin{aligned}
& \frac{X_{1}(\Delta)}{n} \text { converges to zero at the rate } n^{-4 / 3} \text { for any } \Delta>0, \\
& \lim _{\Delta \rightarrow 0} \frac{X_{1}(\Delta)}{n} \text { converges to zero at the rate } n^{-2 / 3} .
\end{aligned}
$$

2. If $\sigma_{D}^{2}=0$, then as $n \rightarrow \infty$ :

$$
\begin{aligned}
& \quad \frac{X_{1}(\Delta)}{n} \text { converges to zero at the rate } n^{-2} \text { for any } \Delta>0, \\
& \lim _{\Delta \rightarrow 0} \frac{X_{1}(\Delta)}{n} \text { converges to zero at the rate } n^{-1} .
\end{aligned}
$$

The convergence rates under $\sigma_{D}^{2}=0$ (i.e. pure private values) are also obtained in the model of Vayanos (1999), who is the first to show that convergence rates differ between discrete-time trading and continuous-time trading. Relative to the results of Vayanos (1999), Proposition 10 reveals that the rate of convergence is slower if traders are subject to adverse selection. For any fixed $\Delta>0$ and as $n \rightarrow \infty$, the inefficiency $X_{1}(\Delta) / n$ vanishes at the rate of $n^{-4 / 3}$ if $\sigma_{D}^{2}>0$, but the corresponding rate is $n^{-2}$ if $\sigma_{D}^{2}=0$. If one first takes the limit of $\Delta \rightarrow 0$, then the convergence rates as $n$ becomes large are $n^{-2 / 3}$ and $n^{-1}$ with and without adverse selection, respectively. (The limiting behavior of the strategic equilibrium as $\Delta \rightarrow 0$ is stated in Appendix B.1.) Interestingly, the asymptotic rates do not depend on the size of $\sigma_{D}^{2}$ but only depend on whether $\sigma_{D}^{2}$ is positive or not. 


\section{Proofs}

\section{C.1 Construction of total signals}

In this appendix we show details of the construction of the total signals in Equation (20). The total signals are subsequently used in the strategies of the competitive benchmark and the strategic equilibrium.

Lemma 3. For any constant $x$, we have:

$$
\begin{aligned}
& \mathbb{E}\left[v_{i, T_{k}} \mid H_{i, T_{k}} \cup\left\{\sum_{j \neq i}\left(x \sum_{l=0}^{k} S_{j, T_{l}}+w_{j, T_{k}}\right)\right\}\right] \\
= & w_{i, T_{k}}+\frac{1 /\left(x^{2} \sigma_{\epsilon}^{2}\right)}{1 /\left(x^{2} \sigma_{D}^{2}\right)+1 /\left(x^{2} \sigma_{\epsilon}^{2}\right)+(n-1) /\left(x^{2} \sigma_{\epsilon}^{2}+\sigma_{w}^{2}\right)} \sum_{l=0}^{k} S_{i, T_{l}} \\
& +\frac{1 /\left(x^{2} \sigma_{\epsilon}^{2}+\sigma_{w}^{2}\right)}{1 /\left(x^{2} \sigma_{D}^{2}\right)+1 /\left(x^{2} \sigma_{\epsilon}^{2}\right)+(n-1) /\left(x^{2} \sigma_{\epsilon}^{2}+\sigma_{w}^{2}\right)} \cdot \frac{1}{x}\left(\sum_{j \neq i}\left(x \sum_{l=0}^{k} S_{j, T_{l}}+w_{j, T_{k}}\right)\right) .
\end{aligned}
$$

Proof. Define

$$
\tilde{S}_{i, T_{l}} \equiv x S_{i, T_{l}}+w_{i, T_{l}}-w_{i, T_{l-1}} .
$$

By the projection theorem for multivariate normal distribution:

$$
\begin{aligned}
& \mathbb{E}\left[D_{T_{l}}-D_{T_{l-1}} \mid S_{i, T_{l}}, \sum_{j \neq i} \tilde{S}_{j, T_{l}}\right] \\
= & \left(x \sigma_{D}^{2},(n-1) x \sigma_{D}^{2}\right) \cdot\left(\begin{array}{lr}
x^{2}\left(\sigma_{D}^{2}+\sigma_{\epsilon}^{2}\right) \\
(n-1) x^{2} \sigma_{D}^{2}
\end{array}\right. \\
& \cdot\left(x S_{i, T_{l}}, \sum_{j \neq i} \tilde{S}_{j, T_{l}}\right)^{\prime} .
\end{aligned}
$$

We compute:

$$
\begin{aligned}
& \left(\begin{array}{cc}
x^{2}\left(\sigma_{D}^{2}+\sigma_{\epsilon}^{2}\right) & (n-1) x^{2} \sigma_{D}^{2} \\
(n-1) x^{2} \sigma_{D}^{2} & (n-1)\left(x^{2}\left(\sigma_{D}^{2}+\sigma_{\epsilon}^{2}\right)+\sigma_{w}^{2}\right)+(n-1)(n-2) x^{2} \sigma_{D}^{2}
\end{array}\right)^{-1} \\
= & \left(\begin{array}{cc}
(n-1)\left(x^{2} \sigma_{\epsilon}^{2}+\sigma_{w}^{2}\right)+(n-1)^{2} x^{2} \sigma_{D}^{2} & -(n-1) x^{2} \sigma_{D}^{2} \\
-(n-1) x^{2} \sigma_{D}^{2} & x^{2}\left(\sigma_{D}^{2}+\sigma_{\epsilon}^{2}\right)
\end{array}\right) \\
& \cdot \frac{1}{(n-1) x^{2}\left(x^{2} \sigma_{\epsilon}^{2}+\sigma_{w}^{2}\right)\left(\sigma_{D}^{2}+\sigma_{\epsilon}^{2}\right)+(n-1)^{2} x^{4} \sigma_{D}^{2} \sigma_{\epsilon}^{2}},
\end{aligned}
$$

and

$$
\begin{aligned}
& \mathbb{E}\left[D_{T_{l}}-D_{T_{l-1}} \mid S_{i, T_{l}}, \sum_{j \neq i} \tilde{S}_{j, T_{l}}\right] \\
= & \frac{(n-1) x^{2} \sigma_{D}^{2}\left(x^{2} \sigma_{\epsilon}^{2}+\sigma_{w}^{2}\right) S_{i, T_{l}}+(n-1) x^{3} \sigma_{D}^{2} \sigma_{\epsilon}^{2} \sum_{j \neq i} \tilde{S}_{j, T_{l}}}{(n-1) x^{2}\left(x^{2} \sigma_{\epsilon}^{2}+\sigma_{w}^{2}\right)\left(\sigma_{D}^{2}+\sigma_{\epsilon}^{2}\right)+(n-1)^{2} x^{4} \sigma_{D}^{2} \sigma_{\epsilon}^{2}} \\
= & \frac{\left(1 / x^{2} \sigma_{\epsilon}^{2}\right) S_{i, T_{l}}+\left(1 /\left(x^{2} \sigma_{\epsilon}^{2}+\sigma_{w}^{2}\right)\right) \frac{1}{x} \sum_{j \neq i} \tilde{S}_{j, T_{l}}}{1 /\left(x^{2} \sigma_{\epsilon}^{2}\right)+1 /\left(x^{2} \sigma_{D}^{2}\right)+(n-1) /\left(x^{2} \sigma_{\epsilon}^{2}+\sigma_{w}^{2}\right)} .
\end{aligned}
$$

Summing the above equation across $l \in\{0,1, \ldots, k\}$ and adding $w_{i, T_{k}}$ gives Equation (76). 
By Equation (76), we have

$$
\begin{aligned}
& \mathbb{E}\left[v_{i, T_{k}} \mid H_{i, T_{k}} \cup\left\{\sum_{j \neq i}\left(\chi \sum_{l=0}^{k} S_{j, T_{l}}+w_{j, T_{k}}\right)\right\}\right] \\
= & w_{i, T_{k}}+\chi \sum_{l=0}^{k} S_{i, T_{l}}+\frac{1 /\left(\chi^{2} \sigma_{\epsilon}^{2}+\sigma_{w}^{2}\right)}{1 /\left(\chi^{2} \sigma_{D}^{2}\right)+1 /\left(\chi^{2} \sigma_{\epsilon}^{2}\right)+(n-1) /\left(\chi^{2} \sigma_{\epsilon}^{2}+\sigma_{w}^{2}\right)} \cdot \frac{1}{\chi}\left(\sum_{j \neq i}\left(\chi \sum_{l=0}^{k} S_{j, T_{l}}+w_{j, T_{k}}\right)\right) \\
= & \alpha s_{i, T_{k}}+\frac{1-\alpha}{n-1} \sum_{j \neq i} s_{j, T_{k}},
\end{aligned}
$$

where in the second line we used the definition of $\chi$ in Equation (19), and in the third line we used the definition of $s_{i, T_{k}}$ in Equation (20), and the definition of $\alpha$ :

$$
\alpha \equiv \frac{1}{1+\frac{(n-1) /\left(\chi^{2} \sigma_{\epsilon}^{2}+\sigma_{w}^{2}\right)}{1 /\left(\chi^{2} \sigma_{D}^{2}\right)+1 /\left(\chi^{2} \sigma_{\epsilon}^{2}\right)+(n-1) /\left(\chi^{2} \sigma_{\epsilon}^{2}+\sigma_{w}^{2}\right)} \cdot \frac{1}{\chi}}=\frac{\chi^{2} \sigma_{\epsilon}^{2}+\sigma_{w}^{2}}{n \chi^{2} \sigma_{\epsilon}^{2}+\sigma_{w}^{2}} .
$$

\section{C.2 Verification of the competitive equilibrium strategy}

The value function of trader $i$, rewritten from Equation (12), is:

$$
\begin{gathered}
\max _{\left\{x_{i, t^{\prime} \Delta}\right\}_{t^{\prime} \geq t}} \sum_{t^{\prime}=t}^{\infty} e^{-r\left(t^{\prime}-t\right) \Delta} \mathbb{E}[ \\
\left.-p_{t^{\prime} \Delta}^{c} \cdot x_{i, t^{\prime} \Delta}\left(p_{t^{\prime} \Delta}^{c}\right) \mid H_{i, t^{\prime} \Delta}, p_{t^{\prime} \Delta}^{c}\right] .
\end{gathered}
$$

The first-order condition of (80) with respect to $x_{i, t \Delta}$ at the competitive equilibrium $\left\{x_{i, t^{\prime} \Delta}^{c}\right\}_{t^{\prime} \geq t}$ is

$$
\begin{gathered}
\mathbb{E}\left[\sum_{t^{\prime}=t}^{\infty} e^{-r\left(t^{\prime}-t\right) \Delta}(\right. \\
\left(1-e^{-r \Delta}\right)\left(v_{i, t^{\prime} \Delta}-\frac{\lambda}{r}\left(z_{i, t^{\prime} \Delta}^{c}+x_{i, t^{\prime} \Delta}^{c}\left(p_{t^{\prime} \Delta}^{c}\right)\right)\right) \frac{\partial\left(z_{i, t^{\prime} \Delta}^{c}+x_{i, t^{\prime} \Delta}^{c}\left(p_{t^{\prime} \Delta}^{c}\right)\right)}{\partial x_{i, t \Delta}^{c}\left(p_{t \Delta}^{c}\right)} \\
\left.\left.-p_{t^{\prime} \Delta}^{c} \frac{\partial x_{i, t^{\prime} \Delta}^{c}\left(p_{t^{\prime} \Delta}^{c}\right)}{\partial x_{i, t \Delta}^{c}\left(p_{t \Delta}^{c}\right)}\right) \mid H_{i, t \Delta}, p_{t \Delta}^{c}\right]=0 .
\end{gathered}
$$

Under the derived strategy $x_{i, t \Delta}^{c}$,

$$
\frac{\partial x_{i, t^{\prime} \Delta}^{c}\left(p_{t^{\prime} \Delta}^{c}\right)}{\partial x_{i, t \Delta}^{c}\left(p_{t \Delta}^{c}\right)}=\left\{\begin{array}{ll}
-1, & \text { if } t^{\prime}=t+1 \\
0, & \text { if } t^{\prime}>t+1
\end{array},\right.
$$

and

$$
\frac{\partial\left(z_{i, t^{\prime} \Delta}^{c}+x_{i, t^{\prime} \Delta}^{c}\left(p_{t^{\prime} \Delta}^{c}\right)\right)}{\partial x_{i, t \Delta}^{c}\left(p_{t \Delta}^{c}\right)}=0, \quad t^{\prime}>t .
$$

So the first-order condition reduces to

$$
\mathbb{E}\left[\left(1-e^{-r \Delta}\right)\left(v_{i, t \Delta}-\frac{\lambda}{r}\left(z_{i, t \Delta}^{c}+x_{i, t \Delta}^{c}\left(p_{t \Delta}^{c}\right)\right)\right)-p_{t \Delta}^{c}+\left(1-e^{-r \Delta}\right) p_{(t+1) \Delta}^{c} \mid H_{i, t \Delta}, p_{t \Delta}^{c}\right]=0
$$


Because the price is a martingale, i.e. $\mathbb{E}\left[p_{(t+1) \Delta}^{c} \mid H_{i, t \Delta}, p_{t \Delta}^{c}\right]=p_{t \Delta}^{c}$, the above equation reduces to the conjecture (15).

\section{C.3 Proof of Proposition 2}

We conjecture that traders use the following linear, symmetric and stationary strategy:

$$
x_{j, t \Delta}\left(p ; s_{j, t \Delta}, z_{j, t \Delta}\right)=a s_{j, t \Delta}-b p+d z_{j, t \Delta}+f Z .
$$

This conjecture implies the market-clearing prices of

$$
p_{t \Delta}^{*}=\frac{a}{n b} \sum_{j=1}^{n} s_{j, t \Delta}+\frac{d+n f}{n b} Z .
$$

Fix a history $H_{i, t \Delta}$ and a realization of $\sum_{j \neq i} s_{j, t \Delta}$. We use the single-deviation principle to construct an equilibrium strategy (85): under the conjecture that other traders $j \neq i$ use strategy (85) in every period $t^{\prime} \geq t$, and that trader $i$ returns to strategy (85) in period $t^{\prime} \geq t+1$, we verify that trader $i$ has no incentive to deviate from strategy (85) in period $t .{ }^{17}$

If trader $i$ uses an alternative demand schedule in period $t$, he faces the residual demand $-\sum_{j \neq i} x_{j, t \Delta}\left(p_{t \Delta}\right)$ and is effectively choosing a price $p_{t \Delta}$ and getting $x_{i, t \Delta}\left(p_{t \Delta}\right)=-\sum_{j \neq i} x_{j, t \Delta}\left(p_{t \Delta}\right)$. Therefore, by differentiating trader $i$ 's expected utility in period $t$ with respect to $p_{t \Delta}$ and evaluating it at $p_{t \Delta}=p_{t \Delta}^{*}$ in Equation (86), we obtain the following first order condition in period $t$ of trader $i$ :

$$
\begin{array}{r}
\mathbb{E}\left[( n - 1 ) b \cdot \left(\left(1-e^{-r \Delta}\right) \sum_{k=0}^{\infty} e^{-r k \Delta} \frac{\partial\left(z_{i,(t+k) \Delta}+x_{i,(t+k) \Delta}^{*}\right)}{\partial x_{i, t \Delta}^{*}}\left(v_{i,(t+k) \Delta}-\frac{\lambda}{r}\left(z_{i,(t+k) \Delta}+x_{i,(t+k) \Delta}^{*}\right)\right)\right.\right. \\
\left.\left.\quad-\sum_{k=0}^{\infty} e^{-r k \Delta} \frac{\partial x_{i,(t+k) \Delta}^{*}}{\partial x_{i, t \Delta}^{*}} p_{(t+k) \Delta}^{*}\right)-\sum_{k=0}^{\infty} e^{-r k \Delta} x_{i,(t+k) \Delta}^{*} \frac{\partial p_{(t+k) \Delta}^{*}}{\partial p_{t \Delta}} \mid H_{i, t \Delta} \cup\left\{\sum_{j \neq i} s_{j, t \Delta}\right\}\right]=0,
\end{array}
$$

where we write $x_{i,(t+k) \Delta}^{*}=x_{i,(t+k) \Delta}\left(p_{(t+k) \Delta}^{*} ; s_{i,(t+k) \Delta}, z_{i,(t+k) \Delta}\right)$ for the strategy $x_{i,(t+k) \Delta}(\cdot)$ defined in Equation (85), and by definition $z_{i,(t+k+1) \Delta}=z_{i,(t+k) \Delta}+x_{i,(t+k) \Delta}^{*}$.

Since all traders follow the conjectured strategy in Equation (85) from period $t+1$ and onwards, we have the following evolution of inventories: for any $k \geq 1$,

$$
\begin{aligned}
z_{i,(t+k) \Delta}+x_{i,(t+k) \Delta}^{*}= & a s_{i,(t+k) \Delta}-b p_{(t+k) \Delta}^{*}+f Z+(1+d) z_{i,(t+k) \Delta} \\
= & \left(a s_{i,(t+k) \Delta}-b p_{(t+k) \Delta}^{*}+f Z\right)+(1+d)\left(a s_{i,(t+k-1) \Delta}-b p_{(t+k-1) \Delta}^{*}+f Z\right) \\
& +\cdots+(1+d)^{k-1}\left(a s_{i,(t+1) \Delta}-b p_{(t+1) \Delta}^{*}+f Z\right)+(1+d)^{k}\left(x_{i, t \Delta}+z_{i, t \Delta}\right) .
\end{aligned}
$$

The evolution of prices and inventories, given by Equations (86) and (88), reveals that by changing the demand or price in period $t$, trader $i$ has the following effects on inventories and prices in period

\footnotetext{
${ }^{17}$ For a description of the single-deviation principle, also called "one-stage deviation principle", see Theorem 4.1 and 4.2 of Fudenberg and Tirole (1991). We can apply their Theorem 4.2 because the payoff function in our model, which takes the form of a "discounted" sum of period-by-period payoffs, satisfies the required "continuity at infinity" condition.
} 
$t+k, k \geq 1:$

$$
\begin{aligned}
& \frac{\partial\left(z_{i,(t+k) \Delta}+x_{i,(t+k) \Delta}^{*}\right)}{\partial x_{i, t \Delta}^{*}}=(1+d)^{k}, \\
& \frac{\partial x_{i,(t+k) \Delta}^{*}}{\partial x_{i, t \Delta}^{*}}=(1+d)^{k-1} d, \\
& \frac{\partial p_{(t+k) \Delta}^{*}}{\partial p_{t \Delta}}=\frac{\partial p_{(t+k) \Delta}^{*}}{\partial x_{i, t \Delta}^{*}}=0 .
\end{aligned}
$$

As we verify later, the equilibrium value of $d$ satisfies $-1<d<0$, so the partial derivatives (89) and (90) converge.

The first order condition (87) simplifies to:

$$
\begin{aligned}
& \mathbb{E}\left[( n - 1 ) b \left(\left(1-e^{-r \Delta}\right) \sum_{k=0}^{\infty} e^{-r k \Delta}(1+d)^{k}\left(v_{i,(t+k) \Delta}-\frac{\lambda}{r}\left(z_{i,(t+k) \Delta}+x_{i,(t+k) \Delta}^{*}\right)\right)\right.\right. \\
& \left.\left.\quad-p_{t \Delta}^{*}-\sum_{k=1}^{\infty} e^{-r k \Delta}(1+d)^{k-1} d p_{(t+k) \Delta}^{*}\right)-x_{i, t \Delta}^{*} \mid H_{i, t \Delta} \cup\left\{\sum_{j \neq i} s_{j, t \Delta}\right\}\right]=0
\end{aligned}
$$

where we have (cf. Lemma 3, Equations (86) and (88)):

$$
\begin{gathered}
\mathbb{E}\left[p_{i,(t+k) \Delta}^{*} \mid H_{i, t \Delta} \cup\left\{s_{j, \tau}\right\}_{j \neq i, 0 \leq \tau \leq t \Delta}\right]=p_{t \Delta}^{*}, \\
\mathbb{E}\left[v_{i,(t+k) \Delta} \mid H_{i, t \Delta} \cup\left\{s_{j, \tau}\right\}_{j \neq i, 0 \leq \tau \leq t \Delta}\right]=\mathbb{E}\left[v_{i, t \Delta} \mid H_{i, t \Delta} \cup\left\{\sum_{j \neq i} s_{j, t \Delta}\right\}\right] \\
=\alpha s_{i, t \Delta}+\frac{1-\alpha}{n-1} \sum_{j \neq i} s_{j, t \Delta}, \\
\mathbb{E}\left[z_{i,(t+k) \Delta}+x_{i,(t+k) \Delta}^{*} \mid H_{i, t \Delta} \cup\left\{s_{j, \tau}\right\}_{j \neq i, 0 \leq \tau \leq t \Delta}\right] \\
=\left(a s_{i, t \Delta}-b p_{t \Delta}^{*}+f Z\right)\left(\frac{1}{-d}-\frac{(1+d)^{k}}{-d}\right)+(1+d)^{k}\left(x_{i, t \Delta}^{*}+z_{i, t \Delta}\right) .
\end{gathered}
$$

Substituting Equations (86), (93), (94) and (95) into the first-order condition (92) and using the notation $\bar{s}_{t \Delta}=\sum_{1 \leq j \leq n} s_{j, t \Delta} / n$, we get:

$$
\begin{aligned}
(n-1) b\left(1-e^{-r \Delta}\right)[ & \frac{1}{1-e^{-r \Delta}(1+d)}\left(\alpha s_{i, t \Delta}+\frac{1-\alpha}{n-1} \sum_{j \neq i} s_{j, t \Delta}-\left(\frac{a}{b} \bar{s}_{t \Delta}+\frac{d+n f}{n b} Z\right)\right) \\
& -\sum_{k=0}^{\infty} \frac{\lambda}{r} e^{-r k \Delta}(1+d)^{k}\left(\frac{1}{-d}-\frac{(1+d)^{k}}{-d}\right)\left(a s_{i, t \Delta}-b\left(\frac{a}{b} \bar{s}_{t \Delta}+\frac{d+n f}{n b} Z\right)+f Z\right) \\
& \left.-\frac{\lambda}{\left(1-e^{-r \Delta}(1+d)^{2}\right) r}\left(x_{i, t \Delta}^{*}+z_{i, t \Delta}\right)\right]-x_{i, t \Delta}^{*}=0 .
\end{aligned}
$$


Rearranging the terms gives:

$$
\begin{aligned}
\left(1+\frac{(n-1) b\left(1-e^{-r \Delta}\right) \lambda}{\left(1-e^{-r \Delta}(1+d)^{2}\right) r}\right) x_{i, t \Delta}^{*} & \\
=(n-1) b\left(1-e^{-r \Delta}\right)[ & \frac{1}{1-e^{-r \Delta}(1+d)}\left(\frac{n \alpha-1}{n-1} s_{i, t \Delta}+\frac{n-n \alpha}{n-1} \bar{s}_{t \Delta}-\frac{a}{b} \bar{s}_{t \Delta}\right) \\
& -\frac{\lambda e^{-r \Delta}(1+d)}{r\left(1-(1+d) e^{-r \Delta}\right)\left(1-(1+d)^{2} e^{-r \Delta}\right)} a\left(s_{i, t \Delta}-\bar{s}_{t \Delta}\right) \\
& -\frac{\lambda}{\left(1-e^{-r \Delta}(1+d)^{2}\right) r} z_{i, t \Delta} \\
& \left.-\left(\frac{1}{1-e^{-r \Delta}(1+d)}\left(\frac{d+n f}{n b}+\frac{\lambda}{r n}\right)-\frac{\lambda}{\left(1-(1+d)^{2} e^{-r \Delta}\right) n r}\right) Z\right]
\end{aligned}
$$

On the other hand, substituting Equation (86) into the conjectured strategy (85) gives:

$$
x_{i, t \Delta}^{*}=a\left(s_{i, 0}-\bar{s}_{0}\right)+d z_{i, 0}-\frac{d}{n} Z .
$$

We match the coefficients in Equation (98) with those in Equation (97). First of all, we clearly have

$$
a=b .
$$

We also obtain two equations for $b$ and $d$ :

$$
\begin{aligned}
& \left(1+\frac{(n-1) b\left(1-e^{-r \Delta}\right) \lambda}{\left(1-e^{-r \Delta}(1+d)^{2}\right) r}\right)=\frac{\left(1-e^{-r \Delta}\right)(n \alpha-1)}{1-e^{-r \Delta}(1+d)}-\frac{(n-1) b\left(1-e^{-r \Delta}\right) e^{-r \Delta}(1+d) \lambda}{\left(1-(1+d) e^{-r \Delta}\right)\left(1-(1+d)^{2} e^{-r \Delta}\right) r}, \\
& \left(1+\frac{(n-1) b\left(1-e^{-r \Delta}\right) \lambda}{\left(1-e^{-r \Delta}(1+d)^{2}\right) r}\right) d=-\frac{(n-1) b\left(1-e^{-r \Delta}\right) \lambda}{\left(1-e^{-r \Delta}(1+d)^{2}\right) r} .
\end{aligned}
$$

There are two solutions to the above system of equations. One of them leads to unbounded inventories, so we drop it. ${ }^{18}$ The other solution leads to converging inventories and is given by

$$
\begin{aligned}
& b=\frac{(n \alpha-1) r}{2(n-1) e^{-r \Delta} \lambda}\left((n \alpha-1)\left(1-e^{-r \Delta}\right)+2 e^{-r \Delta}-\sqrt{(n \alpha-1)^{2}\left(1-e^{-r \Delta}\right)^{2}+4 e^{-r \Delta}}\right), \\
& d=-\frac{1}{2 e^{-r \Delta}}\left((n \alpha-1)\left(1-e^{-r \Delta}\right)+2 e^{-r \Delta}-\sqrt{(n \alpha-1)^{2}\left(1-e^{-r \Delta}\right)^{2}+4 e^{-r \Delta}}\right) .
\end{aligned}
$$

Lastly, matching the coefficient of $Z$ gives:

$$
f=-\frac{d}{n}-\frac{b \lambda}{n r}
$$

Under the condition $n \alpha>2$, we can show that $b>0$ and $-1<d<0$, that is, the demand schedule is downward-sloping in price and the inventories evolutions (89)-(90) converge.

\footnotetext{
${ }^{18}$ This dropped solution to Equation (100) has the property of $(1+d) e^{-r \Delta}<-1$, which leads to an unbounded path of inventories (cf. Equation (88)) and utilities.
} 
By Equation (19), the condition $n \alpha>2$ is equivalent to the condition

$$
\chi^{2}<\frac{(n-2) \sigma_{w}^{2}}{n \sigma_{\epsilon}^{2}}
$$

which is equivalent to the following condition on the fundamentals:

$$
\frac{1}{n / 2+\sigma_{\epsilon}^{2} / \sigma_{D}^{2}}<\sqrt{\frac{n-2}{n}} \frac{\sigma_{w}}{\sigma_{\epsilon}} .
$$

Finally, we verify the second-order condition. Under the linear strategy in Equation (85) with $b>0$, differentiating the first-order condition (87) with respect to $p_{0}$ gives

$$
(n-1) b\left(1-e^{-r \Delta}\right)\left(-\frac{\lambda}{r}(n-1) b \sum_{k=0}^{\infty} e^{-r k \Delta}(1+d)^{2 k}-1\right)-(n-1) b<0 .
$$

This completes the construction of a perfect Bayesian equilibrium.

\section{C.4 Proof of Proposition 3}

Suppose that every trader $i$ use the strategy:

$$
x_{i, t \Delta}(p)=\sum_{T_{l} \leq t \Delta} a_{l} S_{i, T_{l}}+a_{w} w_{i, t \Delta}-b p+d z_{i, t \Delta}+f
$$

where $\left\{a_{l}\right\}_{l \geq 0}, a_{w}, b, d$ and $f$ are constants. We show that for everyone using strategy (107) to be a perfect Bayesian equilibrium (PBE), the constants must be the ones given by Proposition 2. We divide our arguments into two steps.

Step 1. Define $x_{l} \equiv a_{l} / a_{w} .{ }^{19}$ As a first step, we show that if strategy (107) is a symmetric PBE, then we must have $x_{l}=\chi$ for every $l$, where $\chi$ is defined in Equation (19).

Suppose that $(t-1) \Delta \in\left[T_{k^{\prime}}, T_{k^{\prime}+1}\right)$ and $t \Delta \in\left[T_{k}, T_{k+1}\right)$, so there are $k-k^{\prime} \geq 1$ dividend shocks between time $(t-1) \Delta$ and time $t \Delta .{ }^{20}$ Without loss of generality, assume $k^{\prime}=0$. Since all other traders $j \neq i$ are using strategy (107), by computing the difference $p_{t \Delta}^{*}-p_{(t-1) \Delta}^{*}$, trader $i$ can infer from the period- $t$ price the value of

$$
\sum_{j \neq i} \sum_{l=1}^{k} x_{l} S_{j, T_{l}}+w_{j, T_{l}}-w_{j, T_{l-1}}
$$

By the projection theorem for normal distribution, we have

$$
\begin{aligned}
& \mathbb{E}\left[D_{T_{k}}-D_{T_{0}} \mid H_{i, t \Delta} \cup\left\{\sum_{j \neq i} \sum_{T_{l} \leq t \Delta} x_{l} S_{j, T_{l}}+w_{j, T_{l}}-w_{j, T_{l-1}}\right\}\right] \\
= & \mathbb{E}\left[D_{T_{k}}-D_{T_{0}} \mid\left\{S_{i, T_{l}}\right\}_{l=1}^{k} \cup\left\{\sum_{j \neq i} \sum_{l=1}^{k} x_{l} S_{j, T_{l}}+w_{j, T_{l}}-w_{j, T_{l-1}}\right\}\right] \\
= & \mathbf{u} \boldsymbol{\Sigma}^{-1} \cdot\left(S_{i, T_{1}}, \ldots, S_{i, T_{k}}, \sum_{j \neq i} \sum_{l=1}^{k} x_{l} S_{j, T_{l}}+w_{j, T_{l}}-w_{j, T_{l-1}}\right)^{\prime},
\end{aligned}
$$

\footnotetext{
${ }^{19}$ Clearly, we cannot have $a_{w}=0$, since players use their private values in any equilibrium.

${ }^{20} \mathrm{In}$ period $t=0$, we take $D_{T_{-1}}=w_{i, T_{-1}}=0$.
} 
where $\boldsymbol{\Sigma}$ is the covariance matrix of $\left(S_{i, T_{1}}, \ldots, S_{i, T_{k}}, \sum_{j \neq i} \sum_{l=1}^{k} x_{l} S_{j, T_{l}}+w_{j, T_{l}}-w_{j, T_{l-1}}\right):$ for $1 \leq l \leq$ $k+1$ and $1 \leq m \leq k+1$,

$$
\boldsymbol{\Sigma}_{l, m}=\left\{\begin{array}{ll}
\sigma_{D}^{2}+\sigma_{\epsilon}^{2} & 1 \leq l=m \leq k \\
0 & 1 \leq l \neq m \leq k \\
(n-1)\left(\sum_{l^{\prime}=1}^{k} x_{l^{\prime}}^{2}\right)\left(\sigma_{D}^{2}+\sigma_{\epsilon}^{2}\right)+(n-1) k \sigma_{w}^{2} & l=m=k+1 \\
\quad+(n-1)(n-2) \sum_{l^{\prime}=1}^{k} x_{l^{\prime}} \sigma_{D}^{2} & \\
(n-1) x_{l} \sigma_{D}^{2} & 1 \leq l \leq k, m=k+1
\end{array},\right.
$$

and $\boldsymbol{\Sigma}_{k+1, l}=\boldsymbol{\Sigma}_{l, k+1}$. And $\mathbf{u}$ is a row vector of covariances between

$$
\begin{array}{r}
\left(S_{i, T_{1}}, \ldots, S_{i, T_{k}}, \sum_{j \neq i} \sum_{l=1}^{k} x_{l} S_{j, T_{l}}+w_{j, T_{l}}-w_{j, T_{l-1}}\right) \text { and } D_{T_{k}}-D_{T_{0}}: \\
\mathbf{u}=\left(\sigma_{D}^{2}, \ldots, \sigma_{D}^{2},(n-1) \sum_{l=1}^{k} x_{l} \sigma_{D}^{2}\right) .
\end{array}
$$

Therefore, we have

$$
\begin{aligned}
& \mathbb{E}\left[v_{i, t \Delta} \mid H_{i, t \Delta} \cup\left\{\sum_{j \neq i} \sum_{T_{l} \leq t \Delta} x_{l} S_{j, T_{l}}+w_{j, T_{l}}-w_{j, T_{l-1}}\right\}\right] \\
= & w_{i, T_{k}}+\mathbb{E}\left[D_{T_{0}} \mid\left\{S_{i, T_{0}}\right\} \cup\left\{\sum_{j \neq i} x_{0} S_{j, T_{0}}+w_{j, 0}\right\}\right] \\
& +\mathbf{u} \boldsymbol{\Sigma}^{-1} \cdot\left(S_{i, T_{1}}, \ldots, S_{i, T_{k}}, \sum_{j \neq i} \sum_{l=1}^{k} x_{l} S_{j, T_{l}}+w_{j, T_{l}}-w_{j, T_{l-1}}\right)^{\prime} .
\end{aligned}
$$

Since we look for a symmetric equilibrium in which everyone plays strategy (107), trader $i$ 's conditional value in Equation (111) must place a weight of $x_{l}$ on $S_{i, T_{l}}, 1 \leq l \leq k$, which implies that

$$
\mathbf{u} \mathbf{\Sigma}^{-1}=\mathbf{x}
$$

where $\mathbf{x}=\left(x_{1}, \ldots, x_{k}, y\right)$ and $y$ is an arbitrary number. Clearly, Equation (112) is equivalent to

$$
\mathbf{u}=\mathbf{x} \boldsymbol{\Sigma}
$$

which implies (from the first $k$ entries of the row vector)

$$
\sigma_{D}^{2}=x_{l}\left(\sigma_{D}^{2}+\sigma_{\epsilon}^{2}\right)+y(n-1) x_{l} \sigma_{D}^{2}, \quad 1 \leq l \leq k,
$$

i.e.,

$$
x_{1}=\cdots=x_{k}=\frac{\sigma_{D}^{2}}{\sigma_{D}^{2}+\sigma_{\epsilon}^{2}+y(n-1) \sigma_{D}^{2}} .
$$

Now define $x \equiv x_{1}=\cdots=x_{k}$. Applying Lemma 3 to the conditional value in Equation (111) implies that for the conditional value in Equation (111) to place a weight of $x$ on $S_{i, T_{l}}, 1 \leq l \leq k$, we must have $x=\chi$.

Step 2. Given Step 1, we can rewrite the strategy (107) as

$$
x_{i, t \Delta}(p)=a_{w} \cdot \alpha s_{i, t \Delta}-b p+d z_{i, t \Delta}+f,
$$

where $s_{i, t \Delta}$ is the total signal defined in Equation (20) and $\alpha$ is defined in Equation (21). The equilibrium construction in Appendix C.3 then uniquely determines the values of $a_{w}, b, d$ and $f$. This 
concludes the proof of Proposition 3.

\section{C.5 Proof of Proposition 4}

The exponential convergence to efficient allocation follows directly from Equation (34).

Now we prove the comparative statics. We write

$$
\eta \equiv n \alpha-1
$$

and recall that

$$
\begin{aligned}
1+d & =\frac{1}{2 e^{-r \Delta}}\left(\sqrt{(n \alpha-1)^{2}\left(1-e^{-r \Delta}\right)^{2}+4 e^{-r \Delta}}-(n \alpha-1)\left(1-e^{-r \Delta}\right)\right) \\
& =\frac{2}{\sqrt{(n \alpha-1)^{2}\left(1-e^{-r \Delta}\right)^{2}+4 e^{-r \Delta}}+(n \alpha-1)\left(1-e^{-r \Delta}\right)} .
\end{aligned}
$$

We first note that $\frac{\partial(1+d)}{\partial \eta}<0$.

1. The comparative statics with respect to $r$ follow by straightforward calculations showing that $\frac{\partial(1+d)}{\partial r}<0$.

2. As $\sigma_{D}^{2}$ increases, the left-hand side of Equation (19) increases, and hence the solution $\chi$ to Equation (19) increases, which means that $n \alpha$ decreases because according to Equation (21) $n \alpha$ is a decreasing function of $\chi^{2}$. Thus, $\frac{\partial \eta}{\partial \sigma_{D}^{2}}<0$, and $\frac{\partial(1+d)}{\partial \sigma_{D}^{2}}>0$.

3. As $\sigma_{w}^{2}$ increases, the left-hand side of Equation (19) increases, and hence the solution $\chi$ to Equation (19) increases; by Equation (19) this means that $\sigma_{w}^{2} / \chi^{2}$ must increase as well. Thus, $n \alpha$ increases because according to Equation (21) $n \alpha$ is an increasing function of $\sigma_{w}^{2} / \chi^{2}$. Hence, $\frac{\partial \eta}{\partial \sigma_{w}^{2}}>0$ and $\frac{\partial(1+d)}{\partial \sigma_{w}^{2}}<0$.

4. We can rewrite Equation (19) as

$$
\frac{1}{\frac{1}{\alpha}+\frac{\sigma_{\epsilon}^{2}}{\sigma_{D}^{2}}}=\chi
$$

and Equation (21) as

$$
\chi=\sqrt{\frac{1-\alpha}{n \alpha-1}} \frac{\sigma_{w}}{\sigma_{\epsilon}},
$$

and hence

$$
\frac{1}{\frac{n}{\eta+1}+\frac{\sigma_{\epsilon}^{2}}{\sigma_{D}^{2}}}=\sqrt{\frac{n-\eta-1}{n \eta}} \frac{\sigma_{w}}{\sigma_{\epsilon}} .
$$

From Equation (118) is it straightforward to show that $\eta$ must increase with $n$. Thus, $1+d$ decreases in $n$.

5. For the comparative statics with respect to $\Delta$, direction calculation shows that $\partial(1+d) / \partial \Delta<0$. 
And

$$
\begin{aligned}
& \frac{\partial(\log (1+d) / \Delta)}{\partial \Delta}=-\frac{1}{\Delta^{2}}( r \Delta \frac{\eta \sqrt{\eta^{2}\left(e^{r \Delta}-1\right)^{2}+4 e^{r \Delta}}-\eta^{2}\left(e^{r \Delta}-1\right)-2}{\sqrt{\eta^{2}\left(1-e^{-r \Delta}\right)^{2}+4 e^{-r \Delta}}\left(\sqrt{\eta^{2}\left(e^{r \Delta}-1\right)^{2}+4 e^{r \Delta}}-\eta\left(e^{r \Delta}-1\right)\right)} \\
&\left.+\log \left(\frac{1}{2}\left(\sqrt{\eta^{2}\left(e^{r \Delta}-1\right)^{2}+4 e^{r \Delta}}-\eta\left(e^{r \Delta}-1\right)\right)\right)\right)>0 .
\end{aligned}
$$

\section{C.6 Proofs of Propositions 5, 6, 7, 8 and 10}

We first establish some general properties of the equilibrium welfare, before specializing to the optimal trading frequency given scheduled (Appendices C.6.1 and C.6.2) and stochastic (Appendix C.6.3) arrivals of new information, as well as to the rate that inefficiency vanishes as $n \rightarrow \infty$ (Appendix C.6.4).

Proof of Lemma 1. Since $\left(z_{i}\right)^{2}=\left(z_{i,(t+1) \Delta}^{c}\right)^{2}+2 z_{i,(t+1) \Delta}^{c}\left(z_{i}-z_{i,(t+1) \Delta}^{c}\right)+\left(z_{i}-z_{i,(t+1) \Delta}^{c}\right)^{2}$, we have:

$$
\begin{aligned}
& \sum_{i=1}^{n}\left(\left(\alpha s_{i, t \Delta}+\frac{1-\alpha}{n-1} \sum_{j \neq i} s_{j, t \Delta}\right) z_{i}-\frac{\lambda}{2 r}\left(z_{i}\right)^{2}\right) \\
= & \sum_{i=1}^{n}\left(\left(\alpha s_{i, t \Delta}+\frac{1-\alpha}{n-1} \sum_{j \neq i} s_{j, t \Delta}\right) z_{i,(t+1) \Delta}^{c}-\frac{\lambda}{2 r}\left(z_{i,(t+1) \Delta}^{c}\right)^{2}\right) \\
& +\sum_{i=1}^{n}\left(\left(\alpha s_{i, t \Delta}+\frac{1-\alpha}{n-1} \sum_{j \neq i} s_{j, t \Delta}\right)-\frac{\lambda}{r} z_{i,(t+1) \Delta}^{c}\right)\left(z_{i}-z_{i,(t+1) \Delta}^{c}\right) \\
& -\frac{\lambda}{2 r} \sum_{i=1}^{n}\left(z_{i}-z_{i,(t+1) \Delta}^{c}\right)^{2} .
\end{aligned}
$$

The middle term in Equation (119) is zero because

$$
\left(\alpha s_{i, t \Delta}+\frac{1-\alpha}{n-1} \sum_{j \neq i} s_{j, t \Delta}\right)-\frac{\lambda}{r} z_{i,(t+1) \Delta}^{c}=p_{t \Delta}^{c}
$$

for the competitive equilibrium price $p_{t \Delta}^{c}$ (cf. Equations (15) and $\left.(23)\right)$, and $\sum_{i=1}^{n} p_{t \Delta}^{c}\left(z_{i}-z_{i,(t+1)}^{c}\right)=$ $p_{t \Delta}^{c}(Z-Z)=0$. 
Proof of Lemma 2. By Equations (42) and (44), we have

$$
\begin{aligned}
& X_{1}(\Delta) \\
= & \frac{\lambda\left(1-e^{-r \Delta}\right)}{2 r} \sum_{i=1}^{n} \sum_{t=0}^{\infty} e^{-r t \Delta}\left((1+d)^{2(t+1)} \mathbb{E}\left[\left(z_{i, 0}-z_{i, \Delta}^{c}\right)^{2}\right]+\sum_{t^{\prime}=1}^{t}(1+d)^{2\left(t-t^{\prime}+1\right)} \mathbb{E}\left[\left(z_{i,\left(t^{\prime}+1\right) \Delta}^{c}-z_{i, t^{\prime} \Delta}^{c}\right)^{2}\right]\right) \\
= & \frac{\lambda}{2 r} \frac{\left(1-e^{-r \Delta}\right)(1+d)^{2}}{1-(1+d)^{2} e^{-r \Delta}} \sum_{i=1}^{n} \mathbb{E}\left[\left(z_{i, 0}-z_{i, \Delta}^{c}\right)^{2}\right] \\
& +\frac{1-e^{-r \Delta}}{r} \frac{\lambda}{2} \sum_{i=1}^{n} \sum_{t^{\prime}=1}^{\infty} \mathbb{E}\left[\left(z_{i,\left(t^{\prime}+1\right) \Delta}^{c}-z_{i, t^{\prime} \Delta}^{c}\right)^{2}\right] \sum_{t=t^{\prime}}^{\infty} e^{-r t \Delta}(1+d)^{2\left(t-t^{\prime}+1\right)} \\
= & \frac{\lambda}{2 r} \frac{\left(1-e^{-r \Delta}\right)(1+d)^{2}}{1-(1+d)^{2} e^{-r \Delta}} \sum_{i=1}^{n} \mathbb{E}\left[\left(z_{i, 0}-z_{i, \Delta}^{c}\right)^{2}\right] \\
& +\frac{\lambda}{2 r} \frac{\left(1-e^{-r \Delta}\right)(1+d)^{2}}{1-(1+d)^{2} e^{-r \Delta}} \sum_{i=1}^{n} \sum_{t^{\prime}=1}^{\infty} \mathbb{E}\left[\left(z_{i,\left(t^{\prime}+1\right) \Delta}^{c}-z_{i, t^{\prime} \Delta}^{c}\right)^{2}\right] e^{-r t^{\prime} \Delta} .
\end{aligned}
$$

We can simplify the constant in the above equations by a direct calculation:

$$
\begin{aligned}
& e^{-r \Delta}(1+d)^{2} \\
= & \frac{2(n \alpha-1)^{2}\left(1-e^{-r \Delta}\right)^{2}+4 e^{-r \Delta}-2(n \alpha-1)\left(1-e^{-r \Delta}\right) \sqrt{(n \alpha-1)^{2}\left(1-e^{-r \Delta}\right)^{2}+4 e^{-r \Delta}}}{4 e^{-r \Delta}} \\
= & 1-(n \alpha-1)\left(1-e^{-r \Delta}\right)(1+d),
\end{aligned}
$$

which implies:

$$
\frac{\left(1-e^{-r \Delta}\right)(1+d)^{2}}{1-(1+d)^{2} e^{-r \Delta}}=\frac{1+d}{n \alpha-1}
$$

\section{C.6.1 Proof of Proposition 5}

For any $\tau>0$, we let $\bar{t}(\tau)=\min \{t \geq 0: t \in \mathbb{Z}, t \Delta \geq \tau\}$. That is, if new signals arrive at the clock time $\tau$, then $\bar{t}(\tau) \Delta$ is the clock time of the next trading period (including time $\tau$ ).

For any $\Delta \leq \gamma$, by the assumption of Proposition 5 there is at most one new signal profile arrival in each interval $[t \Delta,(t+1) \Delta)$. Thus, we only need to count the changes in competitive allocation between period $\bar{t}((k-1) \gamma)$ and $\bar{t}(k \gamma)$, for $k \in \mathbb{Z}_{+}$. Using this fact, we can rewrite $X_{1}(\Delta)$ and $X_{2}(\Delta)$ as:

$$
\begin{aligned}
X_{1}(\Delta)= & \frac{\lambda(1+d)}{2 r(n \alpha-1)}\left(\sum_{i=1}^{n} \mathbb{E}\left[\left(z_{i, 0}-z_{i, 0}^{e}\right)^{2}\right]+\sum_{i=1}^{n} \sum_{k=1}^{\infty} e^{-r \bar{t}(k \gamma) \Delta} \mathbb{E}\left[\left(z_{i, k \gamma}^{e}-z_{i,(k-1) \gamma}^{e}\right)^{2}\right]\right) \\
= & \frac{\lambda(1+d)}{2 r(n \alpha-1)}\left(\sum_{i=1}^{n} \mathbb{E}\left[\left(z_{i, 0}-z_{i, 0}^{e}\right)^{2}\right]+\sum_{i=1}^{n} \sum_{k=1}^{\infty} e^{-r k \gamma} \mathbb{E}\left[\left(z_{i, k \gamma}^{e}-z_{i,(k-1) \gamma}^{e}\right)^{2}\right]\right) \\
& -\frac{\lambda(1+d)}{2 r(n \alpha-1)} \sum_{i=1}^{n} \sum_{k=1}^{\infty}\left(e^{-r k \gamma}-e^{-r \bar{t}(k \gamma) \Delta}\right) \mathbb{E}\left[\left(z_{i, k \gamma}^{e}-z_{i,(k-1) \gamma}^{e}\right)^{2}\right] .
\end{aligned}
$$


and

$$
\begin{aligned}
X_{2}(\Delta) & =\frac{\lambda}{2 r} \sum_{i=1}^{n} \sum_{t=0}^{\infty} \int_{\tau=t \Delta}^{(t+1) \Delta} r e^{-r \tau} \mathbb{E}\left[\left(z_{i, t \Delta}^{e}-z_{i, \tau}^{e}\right)^{2}\right] d \tau \\
& =\frac{\lambda}{2 r} \sum_{i=1}^{n} \sum_{k=1}^{\infty}\left(e^{-r k \gamma}-e^{-r \bar{t}(k \gamma) \Delta}\right) \mathbb{E}\left[\left(z_{i, k \gamma}^{e}-z_{i,(k-1) \gamma}^{e}\right)^{2}\right] .
\end{aligned}
$$

Note that all the expectations in the expressions of $X_{1}(\Delta)$ and $X_{2}(\Delta)$ do not depend on $\Delta$. To make clear the dependence of $d$ on $\Delta$, we now write $d=d(\Delta)$. Since $(1+d(\Delta)) /(n \alpha-1)<1$, we have for any $\Delta<\gamma$ :

$$
\begin{aligned}
X(\Delta) & =X_{1}(\Delta)+X_{2}(\Delta) \\
& >\frac{\lambda(1+d(\Delta))}{2 r(n \alpha-1)}\left(\sum_{i=1}^{n} \mathbb{E}\left[\left(z_{i, 0}-z_{i, 0}^{e}\right)^{2}\right]+\sum_{i=1}^{n} \sum_{k=1}^{\infty} e^{-r k \gamma} \mathbb{E}\left[\left(z_{i, k \gamma}^{e}-z_{i,(k-1) \gamma}^{e}\right)^{2}\right]\right) \\
& >\frac{\lambda(1+d(\gamma))}{2 r(n \alpha-1)}\left(\sum_{i=1}^{n} \mathbb{E}\left[\left(z_{i, 0}-z_{i, 0}^{e}\right)^{2}\right]+\sum_{i=1}^{n} \sum_{k=1}^{\infty} e^{-r k \gamma} \mathbb{E}\left[\left(z_{i, k \gamma}^{e}-z_{i,(k-1) \gamma}^{e}\right)^{2}\right]\right) \\
& =X(\gamma),
\end{aligned}
$$

where the last inequality holds because $d(\Delta)$ decreases with $\Delta$ (which can be verified by taking derivative the $d^{\prime}(\Delta)$ ) and where the last equality holds because $\bar{t}(k \gamma) \Delta=k \gamma$ if $\gamma=\Delta$. Therefore, we have $W(\Delta)<W(\gamma)$ for any $\Delta<\gamma$. This proves Proposition 5 .

Notice that for this lower bound of $\Delta^{*} \geq \gamma$ we make no use of the assumption that $\mathbb{E}\left[\left(z_{i, k \gamma}^{e}-\right.\right.$ $\left.\left.z_{i,(k-1) \gamma}^{e}\right)^{2}\right]$ is a constant independent of $k$. Thus $\Delta^{*} \geq \gamma$ also holds if traders have no common value but have private value shocks $w_{i, k \gamma}-w_{i,(k-1) \gamma}$ that are non-stationary in $k$.

\section{C.6.2 Proof of Proposition 6}

If $\Delta=l \gamma$, where $l \geq 1$ is an integer, we have:

$$
\begin{aligned}
X_{1}(l \gamma) & =\frac{\lambda(1+d(l \gamma))}{2 r(n \alpha-1)}\left(\sigma_{0}^{2}+\sum_{t=0}^{\infty} e^{-r(t+1) l \gamma} l \sigma_{z}^{2}\right)=\frac{\lambda(1+d(l \gamma))}{2 r(n \alpha-1)}\left(\sigma_{0}^{2}+\frac{e^{-r l \gamma}}{1-e^{-r l \gamma}} l \sigma_{z}^{2}\right) \\
X_{2}(l \gamma) & =\frac{\lambda}{2 r} \frac{1}{1-e^{-r l \gamma}}\left(\left(e^{-\gamma r}-e^{-2 \gamma r}\right)+2\left(e^{-2 \gamma r}-e^{-3 \gamma r}\right)+\cdots+(l-1)\left(e^{-(l-1) \gamma r}-e^{-l \gamma r}\right)\right) \sigma_{z}^{2} \\
& =\frac{\lambda}{2 r} \frac{1}{1-e^{-r l \gamma}}\left(e^{-\gamma r}+e^{-2 \gamma r}+e^{-3 \gamma r}+\cdots+e^{-(l-1) \gamma r}-(l-1) e^{-l \gamma r}\right) \sigma_{z}^{2} \\
& =\frac{\lambda}{2 r} \frac{1}{1-e^{-r l \gamma}}\left(\frac{1-e^{-r l \gamma}}{1-e^{-\gamma r}}-1-(l-1) e^{-r l \gamma}\right) \sigma_{z}^{2} \\
& =\frac{\lambda}{2 r}\left(\frac{1}{1-e^{-\gamma r}}-1-l \frac{e^{-r l \gamma}}{1-e^{-r l \gamma}}\right) \sigma_{z}^{2} .
\end{aligned}
$$

Hence, if $\Delta=l \gamma, l \in \mathbb{Z}_{+}$, we have:

$$
X(l \gamma)=\frac{\lambda(1+d(l \gamma))}{2 r(n \alpha-1)} \sigma_{0}^{2}-\frac{\lambda}{2 r}\left(1-\frac{1+d(l \gamma)}{n \alpha-1}\right) \frac{l e^{-r l \gamma}}{1-e^{-r l \gamma}} \sigma_{z}^{2}+\frac{\lambda e^{-\gamma r}}{2 r\left(1-e^{-\gamma r}\right)} \sigma_{z}^{2}
$$


By taking derivative, we can show that the function (involved in the first term in Equation (129))

$$
\frac{1+d(\Delta)}{n \alpha-1}=\frac{1}{2 e^{-r \Delta}}\left(\sqrt{\left(1-e^{-r \Delta}\right)^{2}+\frac{4 e^{-r \Delta}}{(n \alpha-1)^{2}}}-\left(1-e^{-r \Delta}\right)\right)
$$

is strictly decreasing in $\Delta$, while

$$
\left(1-\frac{1+d(\Delta)}{n \alpha-1}\right) \frac{\Delta e^{-r \Delta}}{1-e^{-r \Delta}}
$$

involved in the second term in Equation (129) is also strictly decreasing in $\Delta$.

We first prove part 2 of Proposition 6. As $n$ tends to infinity, the proof of Proposition 10 implies that $n \alpha$ tends to infinity as well. As $n \alpha \rightarrow \infty,(1+d(l \gamma)) /(n \alpha-1) \rightarrow 0$ for every $l \in \mathbb{Z}_{+}$, and by assumption $\sigma_{0}^{2} / \sigma_{z}^{2}$ remains bounded, so the second term in Equation (129) dominates, and hence $X(l \gamma)$ is minimized at $l^{*}=1$.

For part 1 of Proposition 6 , suppose $z_{i, 0}=Z / n$ for every $i$, so we have $\sigma_{0}^{2}=\sigma_{z}^{2}$. Minimizing $X(l \gamma)$ over positive integers $l$ is equivalent to maximizing $\tilde{W}(l \gamma)$ over $l$ :

$$
\tilde{W}(l \gamma) \equiv \log \left(1-\frac{1+d(l \gamma)}{n \alpha-1}\right)+\log \left(1+\frac{l e^{-r l \gamma}}{1-e^{-r l \gamma}}\right) .
$$

We have:

$$
\tilde{W}=\log \left(1+\delta-\sqrt{(1-\delta)^{2}+4 \delta y}\right)-\log (2 \delta)+\log \left(1-\frac{\log (\delta) \delta}{r \gamma(1-\delta)}\right)
$$

where

$$
\delta \equiv e^{-r l \gamma}, \quad y \equiv \frac{1}{(n \alpha-1)^{2}}
$$

We calculate:

$$
\frac{d \tilde{W}}{d \delta}=\frac{1-\delta-\sqrt{1+\delta(-2+4 y+\delta)}}{2 \delta \sqrt{1+\delta(-2+4 y+\delta)}}+\frac{\delta-1-\log (\delta)}{(1-\delta)(r \gamma(1-\delta)-\delta \log (\delta))} .
$$

Clearly, $\frac{1-\delta-\sqrt{1+\delta(-2+4 y+\delta)}}{2 \delta \sqrt{1+\delta(-2+4 y+\delta)}}$ is decreasing in $y$, and $y \in(0,1)$, so

$$
\frac{1-\delta-\sqrt{1+\delta(-2+4 y+\delta)}}{2 \delta \sqrt{1+\delta(-2+4 y+\delta)}}>-\frac{1}{1+\delta}
$$

where the right-hand side is obtained from substituting $y=1$ to the left-hand side.

In the proof of Proposition 7 (Appendix C.6.3) we have shown that

$$
\frac{d \tilde{W}}{d \delta}>-\frac{1}{1+\delta}+\frac{\delta-1-\log (\delta)}{(1-\delta)(r \gamma(1-\delta)-\delta \log (\delta))}>0
$$

whenever $0 \leq \delta \leq e^{-1.5 r \gamma}$. This proves that the $l^{*}$ that maximizes $\tilde{W}$ (and hence minimizes $X$ ) satisfies $l^{*} \leq 2$. 
To show that $l^{*}=1$, we calculate that:

$\int_{e^{-2 r \gamma}}^{e^{-r \gamma}} \frac{d \tilde{W}}{d \delta} d \delta>\int_{e^{-2 r \gamma}}^{e^{-r \gamma}}-\frac{1}{1+\delta}+\frac{\delta-1-\log (\delta)}{(1-\delta)(r \gamma(1-\delta)-\delta \log (\delta))} d \delta=\left.\log \left(\frac{r \gamma(\delta-1)+\delta \log (\delta)}{1-\delta^{2}}\right)\right|_{e^{-2 r \gamma}} ^{e^{-r \gamma}}=0$

\section{C.6.3 Proofs of Proposition 7 and Proposition 8}

We have:

$$
\begin{aligned}
X_{1}(\Delta) & =\frac{\lambda(1+d)}{2 r(n \alpha-1)}\left(\sigma_{0}^{2}+\sum_{t=0}^{\infty} e^{-r(t+1) \Delta} \Delta \mu \sigma_{z}^{2}\right) \\
& =\frac{\lambda(1+d)}{2 r(n \alpha-1)}\left(\sigma_{0}^{2}+\frac{\Delta e^{-r \Delta}}{1-e^{-r \Delta}} \mu \sigma_{z}^{2}\right)
\end{aligned}
$$

and

$$
X_{2}(\Delta)=\frac{\lambda}{2 r} \sum_{t=0}^{\infty} e^{-r t \Delta} \int_{\tau=0}^{\Delta} r e^{-r \tau} \tau \mu \sigma_{z}^{2} d \tau=-\frac{\lambda}{2 r} \frac{\Delta e^{-r \Delta}}{1-e^{-r \Delta}} \mu \sigma_{z}^{2}+\frac{\lambda}{2 r^{2}} \mu \sigma_{z}^{2} .
$$

Therefore,

$$
X(\Delta)=\frac{\lambda(1+d)}{2 r(n \alpha-1)} \sigma_{0}^{2}-\frac{\lambda}{2 r}\left(1-\frac{1+d}{n \alpha-1}\right) \frac{\Delta e^{-r \Delta}}{1-e^{-r \Delta}} \mu \sigma_{z}^{2}+\frac{\lambda}{2 r^{2}} \mu \sigma_{z}^{2}
$$

We note that the above is the same expression as Equation (129) in the proof of Proposition 6, replacing $\mu$ with $1 / \gamma$ and ignoring the last term which is independent of $\Delta$. The result (Part 2 of Proposition 8) for $n \rightarrow \infty$ has the same proof as that in Proposition 6. Part 1 of Proposition 8 is established by Equation (57) in the main text.

For the proof of Proposition 7, suppose that $z_{i, 0}=Z / n$ for every trader $i$. Then we have $\sigma_{0}^{2}=\sigma_{z}^{2}$. Minimizing $X(\Delta)$ over $\Delta$ is equivalent to maximizing $\tilde{W}(\Delta)$ over $\Delta$, where:

$$
\tilde{W}(\Delta) \equiv \log \left(1-\frac{1+d(\Delta)}{n \alpha-1}\right)+\log \left(1+\frac{\mu \Delta e^{-r \Delta}}{1-e^{-r \Delta}}\right) .
$$

We have:

$$
\tilde{W}=\log \left(1+\delta-\sqrt{(1-\delta)^{2}+4 \delta y}\right)-\log (2 \delta)+\log \left(1-\frac{\mu \log (\delta) \delta}{r(1-\delta)}\right)
$$

where

$$
\delta \equiv e^{-r \Delta}, \quad y \equiv \frac{1}{(n \alpha-1)^{2}}
$$

We calculate:

$$
\frac{d \tilde{W}}{d \delta}=\frac{1-\delta-\sqrt{1+\delta(-2+4 y+\delta)}}{2 \delta \sqrt{1+\delta(-2+4 y+\delta)}}+\frac{\delta-1-\log (\delta)}{(1-\delta)((1-\delta) r / \mu-\delta \log (\delta))},
$$

We note that the first term in the righthand side of Equation (143) is negative, while the second term 
is positive. Moreover,

$$
-\frac{1-\delta-\sqrt{1+\delta(-2+4 y+\delta)}}{2 \delta \sqrt{1+\delta(-2+4 y+\delta)}} / \frac{\delta-1-\log (\delta)}{(1-\delta)((1-\delta) r / \mu-\delta \log (\delta))}
$$

is increasing in $\delta$, tends to 0 as $\delta \rightarrow 0$, and tends to $1+r / \mu$ as $\delta \rightarrow 1$. Therefore, there exists a unique $\delta^{*}$ at which $\frac{d \tilde{W}}{d \delta}=0$.

Equation (143) implies that

$$
\frac{d^{2} \tilde{W}}{d \delta d y}=-\frac{(1-\delta)}{(1+\delta(4 y+\delta-2))^{3 / 2}}<0
$$

for every $\delta \in(0,1)$ and $y \in(0,1)$. Therefore, the optimal $\delta^{*}$ that maximizes $\tilde{W}$ is strictly decreasing with $y$, i.e., the optimal $\Delta^{*}$ that maximizes $\tilde{W}$ is strictly decreasing with $n \alpha$. We have previously established that $\alpha$ is increasing with $\sigma_{w}^{2}$ and is decreasing with $\sigma_{D}^{2}$, and $n \alpha$ is increasing with $n$. This concludes the proof of Part 2 for Proposition 7.

From Equation (143), we have $\frac{d \tilde{W}}{d \delta}>0$ if

$$
y<\frac{(1-\delta)^{2}}{4 \delta}\left(\left(\frac{(1-\delta)((1-\delta) r / \mu-\delta \log (\delta))}{(1-\delta)^{2} r / \mu+2 \delta(1-\delta)+\left(\delta^{2}+\delta\right) \log (\delta)}\right)^{2}-1\right)
$$

After substituting $\delta=e^{-r /(l \mu)}$, the righthand side of the above equation is increasing in $r / \mu$ if $l \geq 2 / 3$. As $r / \mu \rightarrow 0$, the righthand side tends to $9 /(6 l-1)^{2}$. This proves Part 1 of Proposition 7 .

\section{C.6.4 Proofs of Proposition 10}

Suppose that $T_{0}=0$ and $\left\{T_{k}\right\}_{k \geq 1}$ is a homogeneous Poisson process with intensity $\mu>0$. (The proof for scheduled information arrivals $T_{k}=k \gamma$ is analogous and omitted.)

Lemma 2 then implies that

$$
\frac{X_{1}(\Delta)}{n}=\frac{\lambda(1+d(\Delta))}{2 r(n \alpha-1)} \cdot\left(\frac{\sum_{i=1}^{n} \mathbb{E}\left[\left(z_{i, 0}-z_{i, 0}^{e}\right)^{2}\right]}{n}+\frac{e^{-r \Delta} \mu \Delta}{1-e^{-r \Delta}} \frac{\sum_{i=1}^{n} \mathbb{E}\left[\left(z_{i, T_{k}}^{e}-z_{i, T_{k-1}}^{e}\right)^{2}\right]}{n}\right),
$$

where for any $k \geq 1$,

$$
\frac{\sum_{i=1}^{n} \mathbb{E}\left[\left(z_{i, T_{k}}^{e}-z_{i, T_{k-1}}^{e}\right)^{2}\right]}{n}=\left(\frac{r(n \alpha-1)}{\lambda(n-1)}\right)^{2} \frac{(n-1)\left(\chi^{2}\left(\sigma_{D}^{2}+\sigma_{\epsilon}^{2}\right)+\sigma_{w}^{2}\right)}{n \alpha^{2}},
$$

by Equation (46).

Equation (147) tends to a positive constant as $n \rightarrow \infty$ (since $\chi \rightarrow 0$ as $n \rightarrow \infty$ ), and $\lim _{\Delta \rightarrow 0} \frac{e^{-r \Delta} \mu \Delta}{1-e^{-r \Delta}}=$ $\frac{\mu}{r}$. By assumption, $\sum_{i=1}^{n} \mathbb{E}\left[\left(z_{i, 0}-z_{i, 0}^{e}\right)^{2}\right] / n$ is bounded as $n \rightarrow \infty$. Thus, for $\lim _{n \rightarrow \infty} X_{1}(\Delta) / n$ it suffices to analyze

$$
\frac{1+d(\Delta)}{n \alpha-1}=\frac{1}{2 e^{-r \Delta}}\left(\sqrt{\left(1-e^{-r \Delta}\right)^{2}+\frac{4 e^{-r \Delta}}{(n \alpha-1)^{2}}}-\left(1-e^{-r \Delta}\right)\right) .
$$

Suppose $\sigma_{D}^{2}>0$. Equation (118) (where $\eta \equiv n \alpha-1$ ) implies that $n \alpha$ is of order $n^{2 / 3}$ as $n \rightarrow \infty$. To see this, first note that $\eta \rightarrow \infty$ and $\eta / n \rightarrow 0$ as $n \rightarrow \infty$, for otherwise the left-hand side and right-hand side of Equation (118) cannot match. Suppose that as $n$ becomes large, $\eta$ is of order $n^{y}$ 
for some $y<1$. The left-hand side of Equation (118) is of order $n^{y-1}$, and the right-hand side is of order $n^{-y / 2}$. Thus, $y=2 / 3$.

For any fixed $\Delta>0$, it is straightforward to use Taylor expansion to calculate that, as $n$ becomes large,

$$
\frac{1+d(\Delta)}{n \alpha-1}=\frac{1}{1-e^{-r \Delta}}(n \alpha-1)^{-2}+O\left((n \alpha-1)^{-4}\right)
$$

Therefore, $(1+d(\Delta)) /(n \alpha-1)$ and hence $X_{1}(\Delta) / n$ are of order $n^{-4 / 3}$.

But if we first take the limit $\Delta \rightarrow 0$, we clearly have

$$
\lim _{\Delta \rightarrow 0} \frac{1+d(\Delta)}{n \alpha-1}=\frac{1}{n \alpha-1}
$$

so $\lim _{\Delta \rightarrow 0}(1+d(\Delta)) /(n \alpha-1)$ and hence $\lim _{\Delta \rightarrow 0} X_{1}(\Delta) / n$ are of order $n^{-2 / 3}$.

If $\sigma_{D}^{2}=0$, then $n \alpha=n$. The same calculation as above shows that $X_{1}(\Delta) / n$ is of order $n^{-2}$ for a fixed $\Delta>0$ but is of order $n^{-1}$ if we first take the limit $\Delta \rightarrow 0$.

\section{Details of Equilibrium Construction in Section 4}

We fix an arbitrary period $\bar{t}$ and consider a single deviation at that period.

\section{D.1 Fast trader's single deviation}

For a fast trader $i$, raising the price $p_{\bar{t} \Delta}^{*}$ by one unit has the following effects in period $\bar{t}$ :

$$
\frac{\partial x_{i, \bar{t} \Delta}^{*}}{\partial p_{\bar{t} \Delta}^{*}}=\left(n_{F}-1\right) b_{F}+n_{S} b_{S}, \quad \frac{\partial Z_{F, \bar{t} \Delta}}{\partial p_{\bar{t} \Delta}^{*}}=n_{S} b_{S}, \quad \frac{\partial Z_{S, \bar{t} \Delta}}{\partial p_{\bar{t} \Delta}^{*}}=-n_{S} b_{S} .
$$

Fast trader $i$ 's first order condition at period $\bar{t}$ is:

$$
\begin{aligned}
\sum_{t=0}^{\infty} \mathbb{E} & {\left[e ^ { - r t \Delta } \left(-\frac{\lambda_{F}\left(1-e^{-r \Delta}\right)}{r}\left(z_{i,(\bar{t}+t) \Delta}+x_{i,(\bar{t}+t) \Delta}^{*}\right) \frac{\partial\left(z_{i,(\bar{t}+t) \Delta}+x_{i,(\bar{t}+t) \Delta}^{*}\right)}{\partial p_{\bar{t} \Delta}^{*}}-x_{i,(\bar{t}+t) \Delta}^{*} \frac{\partial p_{(\bar{t}+t) \Delta}^{*}}{\partial p_{\bar{t} \Delta}^{*}}\right.\right.} \\
& \left.\left.\quad-p_{(\bar{t}+t) \Delta}^{*} \frac{\partial x_{i,(\bar{t}+t) \Delta}^{*}}{\partial p_{\bar{t} \Delta}^{*}}\right) \mid H_{i, \bar{t} \Delta} \cup\left\{p_{\bar{t} \Delta}^{*}\right\}\right]=0 .
\end{aligned}
$$

For period $\bar{t}+t, t \geq 1$, traders follow the strategies (64) and (65), so we can write:

$$
\begin{aligned}
& \mathbb{E}\left[x_{i,(\bar{t}+t) \Delta}^{*} \mid H_{i, \bar{t} \Delta} \cup\left\{p_{\bar{t} \Delta}^{*}\right\}\right]=C_{x, t, 0}\left(z_{i, \bar{t} \Delta}+x_{i, \bar{t} \Delta}^{*}\right)+\sum_{k=1}^{K-1} C_{x, t, k} Z_{S,(\bar{t}-k) \Delta}+C_{x, t, K} Z_{S, \bar{t} \Delta}+C_{x, t, K+1} Z_{F, \bar{t} \Delta} \\
& \mathbb{E}\left[z_{i,(\bar{t}+t) \Delta} \mid H_{i, \bar{t} \Delta} \cup\left\{p_{\bar{t} \Delta}^{*}\right\}\right]=C_{z, t, 0}\left(z_{i, \bar{t} \Delta}+x_{i, \bar{t} \Delta}^{*}\right)+\sum_{k=1}^{K-1} C_{z, t, k} Z_{S,(\bar{t}-k) \Delta}+C_{z, t, K} Z_{S, \bar{t} \Delta}+C_{z, t, K+1} Z_{F, \bar{t} \Delta} \\
& \mathbb{E}\left[p_{i,(\bar{t}+t) \Delta}^{*} \mid H_{i, \bar{t} \Delta} \cup\left\{p_{\bar{t} \Delta}^{*}\right\}\right]=\sum_{k=1}^{K-1} C_{p, t, k} Z_{S,(\bar{t}-k) \Delta}+C_{p, t, K} Z_{S, \bar{t} \Delta}+C_{p, t, K+1} Z_{F, \bar{t} \Delta,},
\end{aligned}
$$

for each $t \geq 1$. The constants $C_{z, t, k}, C_{x, t, k}$ and $C_{p, t, k}$ can be recursively computed from the coefficients in Equations (64) and (65). 
We can rewrite the first order condition as:

$$
\begin{aligned}
& -x_{i, \bar{t} \Delta}^{*}+\left(-\frac{\lambda_{F}\left(1-e^{-r \Delta}\right)}{r}\left(z_{i, \bar{t} \Delta}+x_{i, \bar{t} \Delta}^{*}\right)-p_{\bar{t} \Delta}^{*}\right)\left(\left(n_{F}-1\right) b_{F}+n_{S} b_{S}\right) \\
& -\frac{\lambda_{F}\left(1-e^{-r \Delta}\right)}{r} \sum_{t=1}^{\infty} e^{-r t \Delta}\left(C_{z, t+1,0}\left(\left(n_{F}-1\right) b_{F}+n_{S} b_{S}\right)-C_{z, t+1, K} n_{S} b_{S}+C_{z, t+1, K+1} n_{S} b_{S}\right) \\
& \cdot\left(C_{z, t+1,0}\left(z_{i, \bar{t} \Delta}+x_{i, \bar{t} \Delta}^{*}\right)+\sum_{k=1}^{K-1} C_{z, t+1, k} Z_{S,(\bar{t}-k) \Delta}+C_{z, t+1, K} Z_{S, \bar{t} \Delta}+C_{z, t+1, K+1} Z_{F, \bar{t} \Delta}\right) \\
& -\sum_{t=1}^{\infty} e^{-r t \Delta}\left(C_{x, t, 0}\left(z_{i, \bar{t} \Delta}+x_{i, \bar{t} \Delta}^{*}\right)+\sum_{k=1}^{K-1} C_{x, t, k} Z_{S,(\bar{t}-k) \Delta}+C_{x, t, K} Z_{S, \bar{t} \Delta}+C_{x, t, K+1} Z_{F, \bar{t} \Delta}\right) \\
& \cdot\left(-C_{p, t, K} n_{S} b_{S}+C_{p, t, K+1} n_{S} b_{S}\right) \\
& -\sum_{t=1}^{\infty} e^{-r t \Delta}\left(\sum_{k=1}^{K-1} C_{p, t, k} Z_{S,(\bar{t}-k) \Delta}+C_{p, t, K} Z_{S, \bar{t} \Delta}+C_{p, t, K+1} Z_{F, \bar{t} \Delta}\right) \\
& \cdot\left(C_{x, t, 0}\left(\left(n_{F}-1\right) b_{F}+n_{S} b_{S}\right)-C_{x, t, K} n_{S} b_{S}+C_{x, t, K+1} n_{S} b_{S}\right) \\
& =0 \text {, }
\end{aligned}
$$

where

$$
\begin{aligned}
Z_{F, \bar{t} \Delta} & =\left(1+d_{F}\right) Z_{F,(\bar{t}-1) \Delta}-n_{F} b_{F} p_{\bar{t} \Delta}^{*}+n_{F} e_{F} Z_{F,(\bar{t}-1) \Delta}+n_{F} \sum_{k=1}^{K-1} f_{F, k} Z_{S,(\bar{t}-k) \Delta}, \\
Z_{S, \bar{t} \Delta} & =\left(1+d_{S}\right) \sum_{j \in \mathcal{C}(\bar{t} \Delta)} z_{j, \bar{t} \Delta}-n_{S} b_{S} p_{\bar{t} \Delta}^{*}+n_{S} e_{S} Z_{F,(\bar{t}-1) \Delta}+n_{S} \sum_{k=1}^{K-1} f_{S, k} Z_{S,(\bar{t}-k) \Delta}, \\
d_{S} \sum_{j \in \mathcal{C}(\bar{t} \Delta)} z_{j, \bar{t} \Delta} & =\left(n_{F} b_{F}+n_{S} b_{S}\right) p_{\bar{t} \Delta}^{*}-\left(\left(d_{F}+n_{F} e_{F}+n_{S} e_{S}\right) Z_{F,(\bar{t}-1) \Delta}+\sum_{k=1}^{K-1}\left(n_{F} f_{F, k}+n_{S} f_{S, k}\right) Z_{S,(\bar{t}-k) \Delta}\right) .
\end{aligned}
$$

\section{D.2 Slow trader's single deviation}

For a slow trader $j$, raising the price $p_{\bar{t} \Delta}^{*}$ by one unit has the following effects in period $\bar{t}$ :

$$
\frac{\partial x_{j, \bar{t} \Delta}^{*}}{\partial p_{\bar{t} \Delta}^{*}}=n_{F} b_{F}+\left(n_{S}-1\right) b_{S}, \quad \frac{\partial Z_{F, \bar{t} \Delta}}{\partial p_{\bar{t} \Delta}^{*}}=-n_{F} b_{F}, \quad \frac{\partial Z_{S, \bar{t} \Delta}}{\partial p_{\bar{t} \Delta}^{*}}=n_{F} b_{F} .
$$

Slow trader $j$ 's first order condition at period $\bar{t}$ is:

$$
\begin{aligned}
\sum_{\substack{t=l K, l \geq 0}} \mathbb{E}\left[e^{-r t \Delta}(\right. & -\frac{\lambda_{S}\left(1-e^{-r K \Delta}\right)}{r}\left(z_{j,(\bar{t}+t) \Delta}+x_{j,(\bar{t}+t) \Delta}^{*}\right) \frac{\partial\left(z_{j,(\bar{t}+t) \Delta}+x_{j,(\bar{t}+t) \Delta}^{*}\right)}{\partial p_{\bar{t} \Delta}^{*}}-x_{j,(\bar{t}+t) \Delta}^{*} \frac{\partial p_{(\bar{t}+t) \Delta}^{*}}{\partial p_{\bar{t} \Delta}^{*}} \\
& \left.\left.-p_{(\bar{t}+t) \Delta}^{*} \frac{\partial x_{j,(\bar{t}+t) \Delta}^{*}}{\partial p_{\bar{t} \Delta}^{*}}\right) \mid H_{j, \bar{t} \Delta} \cup\left\{p_{\bar{t} \Delta}^{*}\right\}\right]=0 .
\end{aligned}
$$


For period $\bar{t}+t$, traders follow the strategies (64) and (65), so we can write:

$$
\begin{aligned}
& \mathbb{E}\left[x_{j,(\bar{t}+t) \Delta}^{*} \mid H_{j, \bar{t} \Delta} \cup\left\{p_{\bar{t} \Delta}^{*}\right\}\right]=D_{x, t, 0}\left(z_{j, \bar{t} \Delta}+x_{j, \bar{t} \Delta}^{*}\right)+\sum_{k=1}^{K-1} D_{x, t, k} Z_{S,(\bar{t}-k) \Delta}+D_{x, t, K} Z_{S, \bar{t} \Delta}+D_{x, t, K+1} Z_{F, \bar{t} \Delta} \\
& \mathbb{E}\left[z_{j,(\bar{t}+t) \Delta} \mid H_{j, \bar{t} \Delta} \cup\left\{p_{\bar{t} \Delta}^{*}\right\}\right]=D_{z, t, 0}\left(z_{j, \bar{t} \Delta}+x_{j, \bar{t} \Delta}^{*}\right)+\sum_{k=1}^{K-1} D_{z, t, k} Z_{S,(\bar{t}-k) \Delta}+D_{z, t, K} Z_{S, \bar{t} \Delta}+D_{z, t, K+1} Z_{F, \bar{t} \Delta},
\end{aligned}
$$

for each $t \in\{K, 2 K, 3 K, \ldots\}$. The constants $D_{z, t, k}$ and $D_{x, t, k}$ can be recursively computed from the coefficients in Equations (64) and (65).

We can rewrite the first order condition as:

$$
\begin{aligned}
& -x_{j, \bar{t} \Delta}^{*}+\left(-\frac{\lambda_{S}\left(1-e^{-r K \Delta}\right)}{r}\left(z_{j, \bar{t} \Delta}+x_{j, \bar{t} \Delta}^{*}\right)-p_{\bar{t} \Delta}^{*}\right)\left(n_{F} b_{F}+\left(n_{S}-1\right) b_{S}\right) \\
& -\frac{\lambda_{S}\left(1-e^{-r K \Delta}\right)}{r} \sum_{\substack{t=l K, l \geq 1}} e^{-r t \Delta}\left(D_{z, t+K, 0}\left(n_{F} b_{F}+\left(n_{S}-1\right) b_{S}\right)+D_{z, t+K, K} n_{F} b_{F}-D_{z, t+K, K+1} n_{F} b_{F}\right) \\
& \cdot\left(D_{z, t+K, 0}\left(z_{j, \bar{t} \Delta}+x_{j, \bar{t} \Delta}^{*}\right)+\sum_{k=1}^{K-1} D_{z, t+K, k} Z_{S,(\bar{t}-k) \Delta}+D_{z, t+K, K} Z_{S, \bar{t} \Delta}+D_{z, t+K, K+1} Z_{F, \bar{t} \Delta}\right) \\
& -\sum_{\substack{t=l K, l \geq 1}} e^{-r t \Delta}\left(D_{x, t, 0}\left(z_{j, \bar{t} \Delta}+x_{j, \bar{t} \Delta}^{*}\right)+\sum_{k=1}^{K-1} D_{x, t, k} Z_{S,(\bar{t}-k) \Delta}+D_{x, t, K} Z_{S, \bar{t} \Delta}+D_{x, t, K+1} Z_{F, \bar{t} \Delta}\right) \\
& \cdot\left(C_{p, t, K} n_{F} b_{F}-C_{p, t, K+1} n_{F} b_{F}\right) \\
& -\sum_{\substack{t=l K, l \geq 1}} e^{-r t \Delta}\left(\sum_{k=1}^{K-1} C_{p, t, k} Z_{S,(\bar{t}-k) \Delta}+C_{p, t, K} Z_{S, \bar{t} \Delta}+C_{p, t, K+1} Z_{F, \bar{t} \Delta}\right) \\
& \cdot\left(D_{x, t, 0}\left(n_{F} b_{F}+\left(n_{S}-1\right) b_{S}\right)+D_{x, t, K} n_{F} b_{F}-D_{x, t, K+1} n_{F} b_{F}\right) \\
& =0 \text {, }
\end{aligned}
$$

where $Z_{F, \bar{t} \Delta}$ and $Z_{S, \bar{t} \Delta}$ are given by Equations (152) and (153).

Given Equations (151) and (157), we then match the coefficients with Equations (64) and (65) to get $2(K+2)$ equations with $2(K+2)$ unknown variables $\left(b_{F}, d_{F}, e_{F}, b_{S}, d_{S}, e_{S}, f_{F, k}\right.$, and $f_{S, k}$, where $k=1, \ldots, K-1)$. 


\section{References}

Ausubel, L. M., P. Cramton, M. Pycia, M. Rostek, and M. Weretka (2014): "Demand Reduction, Inefficiency and Revenues in Multi-Unit Auctions," Review of Economic Studies (Forthcoming).

Biais, B., T. Foucault, And S. Moinas (2015): "Equilibrium Fast Trading," Journal of Financial Economics, 116, 292-313.

Budish, E., P. Cramton, And J. Shim (2015): "The High-Frequency Trading Arms Race: Frequent Batch Auctions as a Market Design Response," Quarterly Journal of Economics, 130, 1547-1621.

Cespa, G. And X. Vives (2013): “The Welfare Impact of High Frequency Trading," Working paper.

CFTC AND SEC (2010): "Findings Regarding the Market Events of May 6, 2010," Report of the Staffs of the CFTC and SEC.

Comerton-Forde, C., A. Frino, And V. Mollica (2005): "The impact of limit order anonymity on liquidity: Evidence from Paris, Tokyo and Korea," Journal of Economics and Business, 57, $528-540$.

Comerton-Forde, C. And K. M. TAng (2009): "Anonymity, liquidity and fragmentation," Journal of Financial Markets, 12, 337-367.

Duffie, D. (2010): "Presidential Address: Asset Price Dynamics with Slow-Moving Capital," Journal of Finance, 65, 1237-1267.

Foster, F. D. And S. Viswanathan (1996): "Strategic Trading When Agents Forecast the Forecasts of Others," Journal of Finance, 51, 1437-1478.

Foucault, T., S. Moinas, And E. Theissen (2007): "Does Anonymity Matter in Electronic Limit Order Markets?" Review of Financial Studies, 20, 1707-1747.

Fudenberg, D. And J. Tirole (1991): Game Theory, MiT Press.

He, H. AND J. WANG (1995): "Differential informational and dynamic behavior of stock trading volume," Review of Financial Studies, 8, 919-972.

Hoffmann, P. (2014): "A dynamic limit order market with fast and slow traders," Journal of Financial Economics, 113, 156-169.

Joint Staff Report (2015): "The U.S. Treasury Market on October 15, 2014," Tech. rep., U.S. Department of the Treasury, Board of Governors of the Federal Reserve System, Federal Reserve Bank of New York, U.S. Securities and Exchange Commission, and U.S. Commodity Futures Trading Commission.

Jovanovic, B. And A. J. Menkveld (2012): "Middlemen in Limit-Order Markets," Working paper.

Kirilenko, A. A., A. S. Kyle, M. Samadi, And T. Tuzun (2015): "The Flash Crash: The Impact of High Frequency Trading on an Electronic Market," Working paper.

KYle, A. S. (1989): "Informed Speculation with Imperfect Competition," Review of Economic Studies, 56, 317-355. 
Kyle, A. S., A. A. Obizhaeva, and Y. Wang (2014): "Smooth Trading with Overconfidence and Market Power," Working paper.

LinnainmaA, J. T. And G. SaAR (2012): "Lack of Anonymity and the Inference from Order Flow," Review of Financial Studies, 25, 1414-1456.

Menkveld, A. J. And B. Z. Yueshen (2015): "The Flash Crash: A Cautionary Tale about Highly Fragmented Markets," Working paper.

Milgrom, P. And C. Shannon (1994): "Monotone Comparative Statics," Econometrica, 62, 157180.

Pagnotta, E. And T. Philippon (2013): "Competing on Speed," Working paper.

Rostek, M. And M. Weretka (2012): "Price Inference in Small Markets," Econometrica, 80, $687-711$.

- (2015): "Dynamic Thin Markets," Review of Financial Studies, 28, 2946-2992.

Sannikov, Y. And A. Skrzypacz (2014): "Dynamic Trading: Price Inertia, Front-Running and Relationship Banking," Working paper.

Securities And Exchange Commission (2010): "Concept Release on Equity Market Structure; Proposed Rule," Federal Register, 75, 3593-3614.

Vayanos, D. (1999): "Strategic Trading and Welfare in a Dynamic Market," The Review of Economic Studies, 66, 219-254.

Vives, X. (2011): "Strategic Supply Function Competition with Private Information," Econometrica, 79, 1919-1966.

WAng, J. (1994): "A Model of Competitive Stock Trading Volume," Journal of Political Economy, $102,127-168$.

Zandt, T. V. (2002): "An Introduction to Monotone Comparative Statics," Tech. rep., INSEAD. 